\title{
Diastereoselective Synthesis of $\gamma$-Lactams by a One-pot, Four-component Reaction
}

Jingqiang Wei and Jared T. Shaw*

The Broad Institute of Harvard and MIT, 7 Cambridge Center, Cambridge, MA 02142

\section{Supporting Information}

General Information, 1-2

Experimental Procedures, 2-22

${ }^{1} \mathrm{H}$ NMR and ${ }^{13} \mathrm{C}$ NMR spectra, 23-73

General Information: All reactions were carried out under an atmosphere of argon in flame dried glassware with magnetic stirring. Dichloromethane $\left(\mathrm{CH}_{2} \mathrm{Cl}_{2}\right)$ and tetrahydrofuran (THF) were purified by passage through a bed of activated alumina. ${ }^{1}$ Purification of reaction products was carried out by flash chromatography using EM Reagent silica gel 60 (230-400 mesh). Analytical thin layer chromatography was performed on EM Reagent $0.25 \mathrm{~mm}$ silica gel $60-\mathrm{F}$ plates. Visualization was accomplished with UV light and aqueous potassium permanganate $\left(\mathrm{KMnO}_{4}\right)$ stain followed by heating. ${ }^{1} \mathrm{H}$ NMR spectra were recorded on a Varian UNITY INOVA500 $(500 \mathrm{MHz})$ spectrometer or a Bruker $300(300 \mathrm{MHz})$ spectrometer. Chemical shifts are reported in ppm relative to the internal standard $\left(\mathrm{CDCl}_{3}: \delta 7.26 \mathrm{ppm} ;\left(\mathrm{CD}_{3}\right)_{2} \mathrm{CO}: \delta 2.05\right.$ ppm). Data are reported as follows: chemical shifts, multiplicity $(\mathrm{br}=$ broad, $\mathrm{s}=$ singlet, $\mathrm{d}$ $=$ doublet, $\mathrm{t}=$ triplet, $\mathrm{q}=$ quartet, $\mathrm{m}=$ multiplet; coupling constant(s) in Hz; integration). Proton-decoupled ${ }^{13} \mathrm{C}$ NMR spectra were recorded on a Varian UNITY INOVA500 (125 $\mathrm{MHz}$ ) spectrometer or a Bruker $300(300 \mathrm{MHz})$ spectrometer. Chemical shifts are reported in ppm using solvent as the internal standard $\left(\mathrm{CDCl} 3\right.$ at $77.0 \mathrm{ppm},\left(\mathrm{CD}_{3}\right)_{2} \mathrm{CO}: \delta$ $29.9 \mathrm{ppm})$. IR frequencies are given in $\mathrm{cm}^{-1}$ and spectra were obtained on a Perkin-Elmer Model 2000 FT-IR spectrophotometer. Tandem high pressure liquid 
chromatography/mass spectral analyses (LCMS) were performed on a Micromass Platform LCZ mass spectrometer or a Micromass Platform LCT mass spectrometer in electrospray ionization (ESI) mode after separation performed on a Waters Alliance 2690 separations module. The actual separation was performed on a Waters Xterra MS C18 $3.5 \mathrm{um}, 4.6 \times 30 \mathrm{~mm}$ column with a flow rate of $3.0 \mathrm{~mL} / \mathrm{min}$ and a $2.5 \mathrm{~min}$ gradient of 5$95 \% \mathrm{CH}_{3} \mathrm{CN}$ in water, constant $0.01 \%$ formic acid buffer using a Waters 996 photodiode array detector.

\section{Experimental Procedures:}
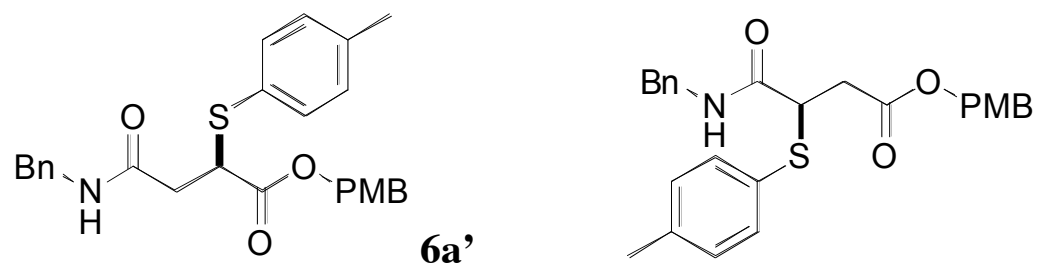

To 3- (p-tolylthio) succinic anhydride $2(0.45 \mathrm{~g}, 2.0 \mathrm{mmol})$ in acetone $(20 \mathrm{~mL})$ at room temperature was added benzylamine $(0.22 \mathrm{~g}, 2.0 \mathrm{mmol})$. After stirring 10 minutes at room temperature, $\mathrm{K}_{2} \mathrm{CO}_{3}(0.28 \mathrm{~g}, 2.0 \mathrm{mmol})$, $\mathrm{KI}(0.33 \mathrm{~g}, 2.0 \mathrm{mmol})$, and $p$ methoxybenzylchloride $(0.31 \mathrm{~g}, 2.0 \mathrm{mmol})$ were added to above solution. The mixture was stirred at room temperature overnight. The solution was concentrated in vacuo and extracted with water and ethyl acetate $(20 \mathrm{~mL} \times 3)$. The organic layers were washed with brine, dried with anhydrous $\mathrm{MgSO}_{4}$. Concentration in vacuo and purification by flash column chromatography (hexane: ethyl acetate $=4: 1$ ) gave pure $\mathbf{6 a}$ ' as light yellow solids $(0.17 \mathrm{~g}, 19 \%)$, pure $\mathbf{6 b}$ ' as light yellow solid (0.26 g, 29\%), and 6a', $\mathbf{6} \mathbf{b}^{\prime}$ mixture as light yellow solids $(0.5 \mathrm{~g}, 55 \%)$.

6a': ${ }^{1} \mathrm{H}$ NMR (500 MHz, $\left.\mathrm{CDCl}_{3}\right)$ d 7.28-7.32 (m, 2H), 7.22-7.27 (m, 5H), 7.18-7.21 (m, 2H), $7.04(\mathrm{~d}, J=8 \mathrm{~Hz}, 2 \mathrm{H}), 6.85-6.87(\mathrm{~m}, 2 \mathrm{H}), 6.20$ (t, $J=5.5 \mathrm{~Hz}, 1 \mathrm{H}, \mathrm{NH}), 5.08$ (d, $J=$ 12, 1H), $4.96(\mathrm{~d}, J=12 \mathrm{~Hz}, 1 \mathrm{H}), 4.39(\mathrm{dd}, J=15,5.5 \mathrm{~Hz}, 1 \mathrm{H}), 4.33(\mathrm{dd}, J=15,5.5 \mathrm{~Hz}$, 
$1 \mathrm{H}), 4.11(\mathrm{dd}, J=9.5,5.5 \mathrm{~Hz}, 1 \mathrm{H}), 3.80(\mathrm{~s}, 3 \mathrm{H}), 2.75(\mathrm{dd}, J=15,6 \mathrm{~Hz}, 1 \mathrm{H}), 2.59$ (dd, $J$ $=15,6 \mathrm{~Hz}, 1 \mathrm{H}), 2.32(\mathrm{~s}, 3 \mathrm{H}) ;{ }^{13} \mathrm{C} \mathrm{NMR}\left(125 \mathrm{MHz}, \mathrm{CDCl}_{3}\right) \delta 171.4,169.2,159.4,138.7$, 137.9, 134.3, 130.0, 129.6, 128.5, 127.9, 127.6, 127.4, 127.3, 113.6, 66.7, 55.1, 46.1, 43.4, 38.0, 21.0; IR (thin film): 3308 (br), 1730, 1649, 1515, 1248, $1155 \mathrm{~cm}^{-1}$; LCMS: mass calcd for $\left(\mathrm{C}_{26} \mathrm{H}_{27} \mathrm{NO}_{4} \mathrm{~S}+\mathrm{H}\right)^{+} \mathrm{m} / z 450.17$, found $\mathrm{m} / z 450.1392, \mathrm{R}_{\mathrm{T}}=1.78 \mathrm{~min}$.

6b': ${ }^{1} \mathrm{H}$ NMR (500 MHz, $\left.\mathrm{CDCl}_{3}\right) \delta$ 7.22-7.30 (m, 6H), $7.18(\mathrm{~m}, 2 \mathrm{H}), 7.03(\mathrm{~d}, J=8 \mathrm{~Hz}$, 2H), 6.83-6.88 (m, 3H), $5.07(\mathrm{~d}, J=12 \mathrm{~Hz}, 1 \mathrm{H}), 5.01(\mathrm{~d}, J=12 \mathrm{~Hz}, 1 \mathrm{H}), 4.42(\mathrm{dd}, J=$ 15, $6 \mathrm{~Hz}, 1 \mathrm{H}), 4.34(\mathrm{dd}, J=15,6 \mathrm{~Hz}, 1 \mathrm{H}), 3.98(t, J=7.5 \mathrm{~Hz}, 1 \mathrm{H}), 3.76(\mathrm{~s}, 3 \mathrm{H}), 3.10$ (dd, $J=17,7.5 \mathrm{~Hz}, 1 \mathrm{H}), 2.74(\mathrm{dd}, J=17,6.5 \mathrm{~Hz}), 2.30(\mathrm{~s}, 3 \mathrm{H}) ;{ }^{13} \mathrm{C} \mathrm{NMR}\left(125 \mathrm{MHz}, \mathrm{CDCl}_{3}\right)$ ठ 170.7, 169.6, 159.4, 138.4, 137.7, 133.2, 129.9, 129.8, 128.4, 128.2, 127.54, 127.50, 127.2, 113.7, 66.3, 55.0, 47.8, 43.7, 36.5, 20.9; IR (thin film): 3306 (br), 1731, 1654, 1514, 1247, 1174, $810 \mathrm{~cm}^{-1}$; LCMS: mass calcd for $\left(\mathrm{C}_{26} \mathrm{H}_{27} \mathrm{NO}_{4} \mathrm{~S}+\mathrm{H}\right)^{+} \mathrm{m} / z$ 450.17, found $m / z 450.1387, \mathbf{R}_{\mathrm{T}}=1.76 \mathrm{~min}$.
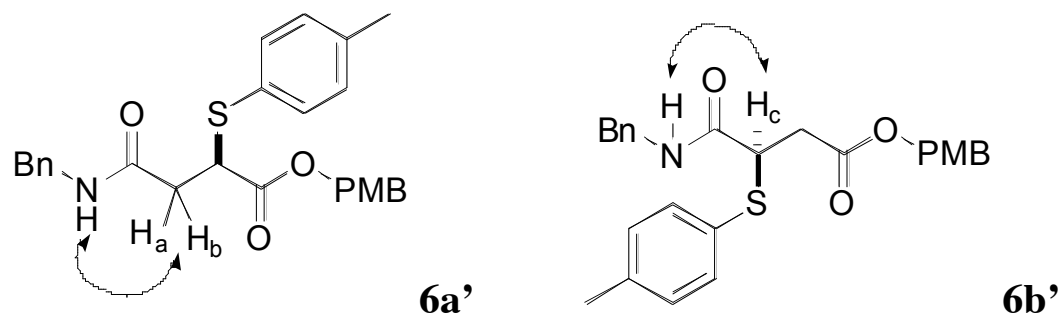

2D-NOE experiments were performed in $\mathrm{CDCl}_{3}$. 6a' was assigned by the coupling between $\mathrm{NH}(\delta 6.20)$ and $\mathrm{H}_{\mathrm{a}}, \mathrm{H}_{\mathrm{b}}(\delta 2.75,2.59)$. 6b' was assigned by the coupling between $\mathrm{NH}(\delta 7.25)$ and $\mathrm{H}_{\mathrm{c}}(\delta 3.98)$. 


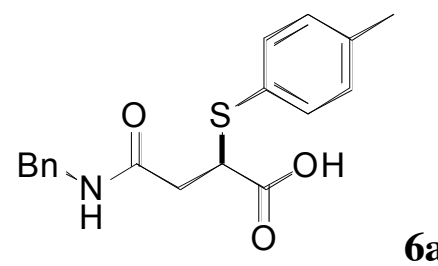

Compound 6a' $(0.17 \mathrm{~g}, 0.38 \mathrm{mmol})$ in $\mathrm{CH}_{2} \mathrm{Cl}_{2}(20 \mathrm{~mL})$ was added trifluoro acetic acid (TFA) $(2 \mathrm{~mL})$ at $0{ }^{\circ} \mathrm{C}$. The solution was warmed up slowly and stirred at room temperature for 5 hours. Evaporation in vacuo removed most solvent. Addition of hexane generated white precipitates, which were collected by filtration and washed three time by hexane to give light gray solids $6 \mathbf{6}(0.12 \mathrm{~g}, 100 \%) .{ }^{1} \mathrm{H} \mathrm{NMR}\left(500 \mathrm{MHz}, \mathrm{CDCl}_{3}\right) \delta$ $7.39(\mathrm{~d}, J=8 \mathrm{~Hz}, 2 \mathrm{H}), 7.33(\mathrm{~m}, 2 \mathrm{H}), 7.26-7.28(\mathrm{~m}, 3 \mathrm{H}), 7.12(\mathrm{~d}, J=8 \mathrm{~Hz}, 2 \mathrm{H}), 5.85$ (br, 1H, NH), $4.44(\mathrm{~d}, J=5.5 \mathrm{~Hz}, 2 \mathrm{H}), 4.10(\mathrm{dd}, J=8.5,5 \mathrm{~Hz}, 1 \mathrm{H}), 3.77(\mathrm{br}, 1 \mathrm{H}, \mathrm{OH}), 2.79$ (dd, $J=15.5,8.5 \mathrm{~Hz}, 1 \mathrm{H}), 2.71(\mathrm{dd}, J=15.5,5 \mathrm{~Hz}, 1 \mathrm{H}), 2.34(\mathrm{~s}, 3 \mathrm{H}) ;{ }^{13} \mathrm{C} \mathrm{NMR}(75 \mathrm{MHz}$, $\left.\mathrm{CDCl}_{3}\right) \delta 173.8,170.2,139.2,137.4,134.2,130.0,128.8,127.9,127.8,46.7,44.1,38.1$, 21.2; IR (thin film): 3294 (br), 1708, 1633, 1494, 1242, 1164, 1030, 809, $698 \mathrm{~cm}^{-1}$; LCMS: mass calcd for $\left(\mathrm{C}_{18} \mathrm{H}_{19} \mathrm{NO}_{3} \mathrm{~S}+\mathrm{H}\right)^{+} \mathrm{m} / z$ 330.11, found $\mathrm{m} / z$ 330.0591, $\mathrm{R}_{\mathrm{T}}=1.46 \mathrm{~min}$.

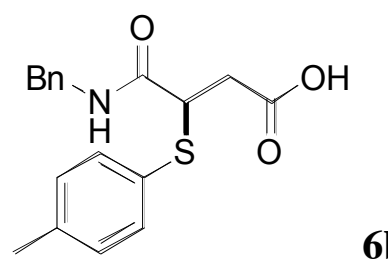

Compound $\mathbf{6 b}$ ' $(0.12 \mathrm{~g}, 0.27 \mathrm{mmol})$ in $\mathrm{CH}_{2} \mathrm{Cl}_{2}(30 \mathrm{~mL})$ was added trifluoro acetic acid (TFA) $(3 \mathrm{~mL})$ at room temperature. The solution was stirred at room temperature overnight. Evaporation in vacuo removed most solvent. Addition of hexane generated white precipitates, which were collected by filtration and washed three time by hexane to give white solids $6 \mathbf{b}(71 \mathrm{mg}, 80 \%) .{ }^{1} \mathrm{H}$ NMR $\left(500 \mathrm{MHz}, \mathrm{CDCl}_{3}\right) \delta 7.82(\mathrm{br}, 1 \mathrm{H}, \mathrm{NH})$, $7.37(\mathrm{~d}, J=8 \mathrm{~Hz}, 2 \mathrm{H}), 7.29(\mathrm{~m}, 4 \mathrm{H}), 7.24(\mathrm{~m}, 1 \mathrm{H}), 7.14(\mathrm{~d}, J=8 \mathrm{~Hz}, 2 \mathrm{H}), 4.44$ (dd, $J=$ $15,6 \mathrm{~Hz}, 1 \mathrm{H}), 4.38(\mathrm{dd}, J=15,6 \mathrm{~Hz}, 1 \mathrm{H}), 4.06(\mathrm{dd}, J=9,5 \mathrm{~Hz}, 1 \mathrm{H}), 3.01(\mathrm{dd}, J=12$, 
$8.5 \mathrm{~Hz}, 1 \mathrm{H}), 2.67(\mathrm{dd}, J=12,5.5 \mathrm{~Hz}, 1 \mathrm{H}), 2.32(\mathrm{~s}, 3 \mathrm{H}), 2.06(\mathrm{~m}, 1 \mathrm{H}) ;{ }^{13} \mathrm{C}$ NMR $(125$ $\left.\mathrm{MHz},\left(\mathrm{CD}_{3}\right)_{2} \mathrm{CO}\right) \delta$ 172.2, 170.8, 140.2, 139.3, 134.8, 130.7, 130.1, 129.2, 128.4, 127.8, 48.1, 43.9, 37.2, 21.2; IR (thin film): 3307 (br), 1714, $1652 \mathrm{~cm}^{-1}$; LCMS: mass calcd for $\left(\mathrm{C}_{18} \mathrm{H}_{19} \mathrm{NO}_{3} \mathrm{~S}+\mathrm{H}\right)^{+} \mathrm{m} / \mathrm{z} 330.11$, found $\mathrm{m} / \mathrm{z} 330.0854, \mathrm{R}_{\mathrm{T}}=1.43 \mathrm{~min}$.

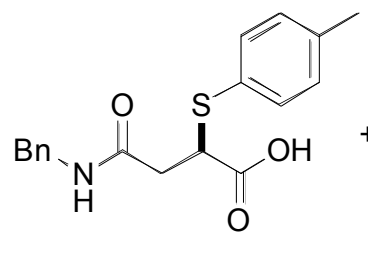

6a

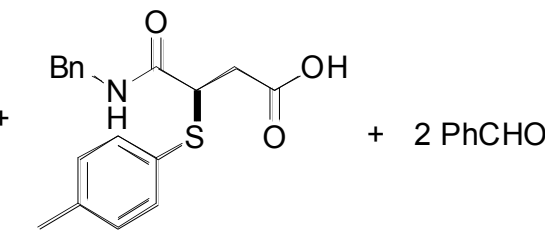

6b

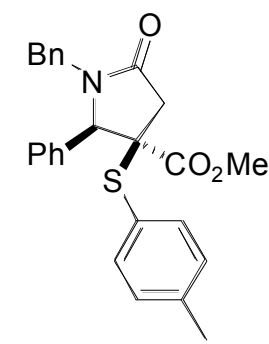

11

To 3- (p-tolylthio) succinic anhydride $2(0.23 \mathrm{~g}, 1.0 \mathrm{mmol})$ in toluene $(10 \mathrm{~mL})$ at room temperature was added benzylamine $(0.11 \mathrm{~g}, 1.0 \mathrm{mmol})$. After stirring 10 minutes at room temperature, reaction was complete to give $\mathbf{6 a}$ and $\mathbf{6 b}$ in 1:1 mixture. To the above mixture, benzaldehyde $(0.11 \mathrm{~g}, 1.0 \mathrm{mmol})$ was added. The mixture was heated to $150{ }^{\circ} \mathrm{C}$ in a sealed tube for 24 hours. After cooled to room temperature, the solution was concentrated in vасио. The residue was dissolved by acetone $(10 \mathrm{~mL})$, followed by addition of anhydrous $\mathrm{K}_{2} \mathrm{CO}_{3}(0.55 \mathrm{~g}, 4.0 \mathrm{mmol})$ and $\mathrm{MeI}(0.57 \mathrm{~g}, 4.0 \mathrm{mmol})$ at $0{ }^{\circ} \mathrm{C}$. The mixture was warmed up to room temperature slowly and stirred overnight. The solution was concentrated in vacuo and extracted with water and ethyl acetate $(20 \mathrm{~mL} \times 3)$. The organic layers were washed with brine, dried with anhydrous $\mathrm{MgSO}_{4}$. Concentration in vacuo and purification by flash column chromatography gave $\mathbf{1 1}$ as white solids $(0.38$ g, 90\%). 
2. Mel, $\mathrm{K}_{2} \mathrm{CO}_{3}$

$6 a$

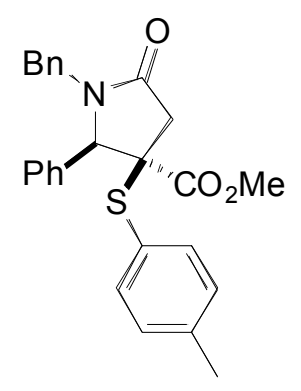

11

Compound 6a $(0.12 \mathrm{~g}, 0.36 \mathrm{mmol})$ and benzaldehyde $(39 \mathrm{mg}, 0.36 \mathrm{mmol})$ in toluene (5 $\mathrm{mL}$ ) was heated to $140{ }^{\circ} \mathrm{C}$ in a sealed tube for 24 hours. After cooled to room temperature, the solution was concentrated in vacuo. The residue was dissolved by acetone $(10 \mathrm{~mL})$, followed by addition of anhydrous $\mathrm{K}_{2} \mathrm{CO}_{3}(0.22 \mathrm{~g}, 1.6 \mathrm{mmol})$ and $\mathrm{MeI}(0.23 \mathrm{~g}, 1.6 \mathrm{mmol})$ at $0{ }^{\circ} \mathrm{C}$. The mixture was warmed up to room temperature slowly and stirred overnight. The solution was concentrated in vacuo and extracted with water and ethyl acetate (10 $\mathrm{mL} \times 3$ ). The organic layers were washed with brine, dried with anhydrous $\mathrm{MgSO}_{4}$. Concentration in vacuo and purification by flash column chromatography gave $\mathbf{1 1}$ as white solids $(0.13 \mathrm{~g}, 81 \%)$.

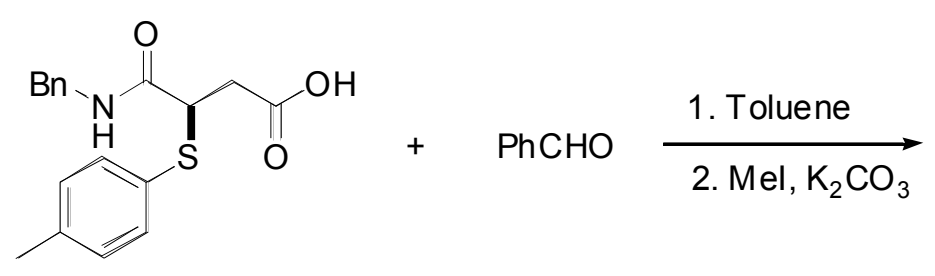

6b

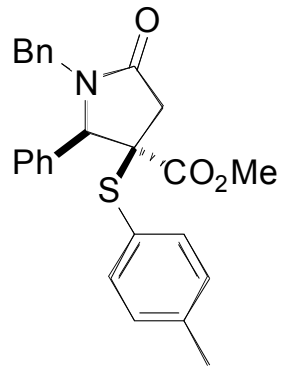

11

Compound 6b (47 mg, $0.14 \mathrm{mmol})$ and benzaldehyde (21 mg, $0.14 \mathrm{mmol})$ in toluene (5 $\mathrm{mL}$ ) was heated to $150{ }^{\circ} \mathrm{C}$ in a sealed tube for 24 hours. After cooled to room temperature, the solution was concentrated in vacuo. The residue was dissolved by acetone $(10 \mathrm{~mL})$, followed by addition of anhydrous $\mathrm{K}_{2} \mathrm{CO}_{3}(41 \mathrm{mg}, 0.3 \mathrm{mmol})$ and $\mathrm{MeI}(43 \mathrm{mg}, 0.3 \mathrm{mmol})$ 
at $0{ }^{\circ} \mathrm{C}$. The mixture was warmed up to room temperature slowly and stirred overnight. The solution was concentrated in vacuo and extracted with water and ethyl acetate (10 $\mathrm{mL} \times 3)$. The organic layers were washed with brine, dried with anhydrous $\mathrm{MgSO}_{4}$. Concentration in vacuo and purification by flash column chromatography gave $\mathbf{1 1}$ as white solids (46 mg, 78\%).

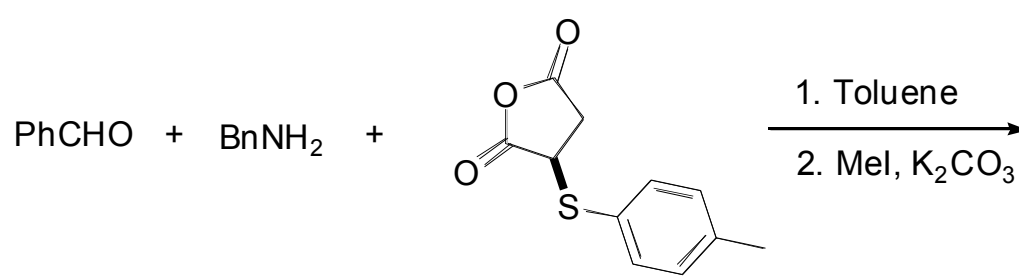

2

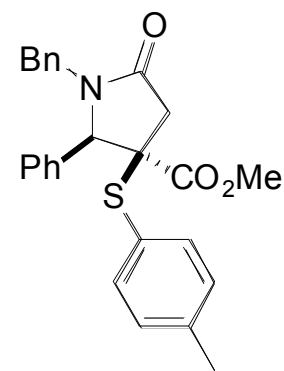

11

Three-component reaction to $\gamma$-lactam: To 3- ( $p$-tolylthio) succinic anhydride $2(0.23 \mathrm{~g}$, $1.0 \mathrm{mmol})$ in toluene $(10 \mathrm{~mL})$ at room temperature was added benzaldehyde $(0.106 \mathrm{~g}, 1.0$ mmol $)$ and benzylamine $(0.11 \mathrm{~g}, 1.0 \mathrm{mmol})$. The mixture was heated to $150{ }^{\circ} \mathrm{C}$ in a sealed tube for 24 hours. After cooled to room temperature, the solution was concentrated in vacuo. The residue was dissolved in acetone $(20 \mathrm{~mL})$, followed by addition of anhydrous $\mathrm{K}_{2} \mathrm{CO}_{3}(0.55 \mathrm{~g}, 4.0 \mathrm{mmol})$ and $\mathrm{MeI}(0.57 \mathrm{~g}, 4.0 \mathrm{mmol})$ at $0{ }^{\circ} \mathrm{C}$. The mixture was warmed up to room temperature slowly and stirred overnight. The solution was concentrated in vacuo and extracted with water and ethyl acetate $(15 \mathrm{~mL} \times 3)$. The organic layers were washed with brine, dried with anhydrous $\mathrm{MgSO}_{4}$. Concentration in vacuo and purification by flash column chromatography (hexane: ethyl acetate $=5: 1$ ) gave white solids $(0.38 \mathrm{~g}, 89 \%)$.

\section{General procedure for synthesis of tetrasubsitituted $\boldsymbol{\gamma}$-lactams by four-component}

reactions: To a solution of maleic anhydride $(1.0 \mathrm{mmol})$, thiol $(1.0 \mathrm{mmol})$, and aldehyde $(1.0 \mathrm{mmol})$ in toluene $(15 \mathrm{~mL})$ was added amine $(1.0 \mathrm{mmol})$ at room temperature. The mixture was refluxed under argon with dean stark for 24 hours. After cooled to room 
temperature, the solution was concentrated in vacuo. The residue was dissolved in acetone $(20 \mathrm{~mL})$, followed by addition of anhydrous $\mathrm{K}_{2} \mathrm{CO}_{3}(0.55 \mathrm{~g}, 4.0 \mathrm{mmol})$ and MeI $(0.57 \mathrm{~g}, 4.0 \mathrm{mmol})$ at $0{ }^{\circ} \mathrm{C}$. The mixture was warmed up to room temperature slowly and stirred overnight. The solution was concentrated in vacuo and extracted with water and ethyl acetate $(15 \mathrm{~mL} \times 3)$. The organic layers were washed with brine, dried with anhydrous $\mathrm{MgSO}_{4}$. Concentration in vacuo and purification by flash column chromatography gave final products. If necessary, preparative TLC plates were used for purification.

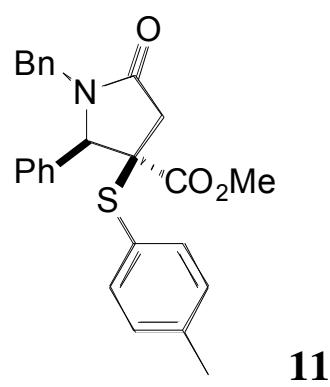

4CR method 1: 11 was prepared from maleic anhydride (98 mg, $1.0 \mathrm{mmol}$ ), $p$-thiocresol $(0.124 \mathrm{~g}, 1.0 \mathrm{mmol})$, benzaldehyde $(0.106 \mathrm{~g}, 1.0 \mathrm{mmol})$, and benzylamine $(0.107 \mathrm{~g}, 1.0$ mmol). Purification on $\mathrm{SiO}_{2}$ (hexane: ethyl acetate $\left.=5: 1\right)$ gave white solids $(0.39 \mathrm{~g}, 91 \%)$. 4CR method 2: Maleic anhydride (50 mg, $0.5 \mathrm{mmol}), p$-thiocresol $(62 \mathrm{mg}, 0.5 \mathrm{mmol})$, benzaldehyde dimethyl acetal ( $76 \mathrm{mg}, 0.5 \mathrm{mmol})$, and benzylamine $(53 \mathrm{mg}, 0.5 \mathrm{mmol})$ in toluene $(10 \mathrm{~mL})$ were heated to $150{ }^{\circ} \mathrm{C}$ in a sealed tube for 24 hours. After cooled to room temperature, the solution was concentrated in vacuo. The residue was dissolved by acetone $(10 \mathrm{~mL})$, followed by addition of anhydrous $\mathrm{K}_{2} \mathrm{CO}_{3}(0.28 \mathrm{~g}, 2.0 \mathrm{mmol})$ and MeI $(0.29 \mathrm{~g}, 2.0 \mathrm{mmol})$ at $0{ }^{\circ} \mathrm{C}$. The mixture was warmed up to room temperature slowly and stirred overnight. The solution was concentrated in vacuo and extracted with water and ethyl acetate $(15 \mathrm{~mL} \times 3)$. The organic layers were washed with brine, dried with 
anhydrous $\mathrm{MgSO}_{4}$. Concentration in vacuo and purification by flash column chromatography (hexane: ethyl acetate $=5: 1)$ gave white solids $(0.11 \mathrm{~g}, 53 \%)$.

${ }^{1} \mathrm{H}$ NMR $\left(300 \mathrm{MHz}, \mathrm{CDCl}_{3}\right) \delta 7.45(\mathrm{~m}, 3 \mathrm{H}), 7.26(\mathrm{~m}, 7 \mathrm{H}), 7.06(\mathrm{~m}, 3 \mathrm{H}), 7.00(\mathrm{~m}, 1 \mathrm{H})$, $5.17(\mathrm{~d}, J=15 \mathrm{~Hz}, 1 \mathrm{H}), 4.92(\mathrm{~s}, 1 \mathrm{H}), 3.53(\mathrm{~s}, 3 \mathrm{H}), 3.49$ (d, $J=15 \mathrm{~Hz}, 1 \mathrm{H}), 3.23(\mathrm{~d}, J=$ $16.8 \mathrm{~Hz}, 1 \mathrm{H}), 2.89$ (d, $J=16.8 \mathrm{~Hz}, 1 \mathrm{H}), 2.30(\mathrm{~s}, 3 \mathrm{H}) ;{ }^{13} \mathrm{C} \mathrm{NMR}\left(125 \mathrm{MHz}, \mathrm{CDCl}_{3}\right) \delta$ $171.7,171.5,139.9,135.9,135.5,133.8,129.6,129.0,128.4,128.4,127.9,127.5,126.0$, 66.7, 58.5, 52.7, 44.4, 40.6, 21.2; IR (thin film): 1731, 1701, 1409, 1247, $702 \mathrm{~cm}^{-1}$; LCMS: mass calcd for $\left(\mathrm{C}_{26} \mathrm{H}_{25} \mathrm{NO}_{3} \mathrm{~S}+\mathrm{H}\right)^{+} m / z$, 432.16, found $m / z, 432.1200, \mathrm{R}_{\mathrm{T}}=1.81 \mathrm{~min}$.

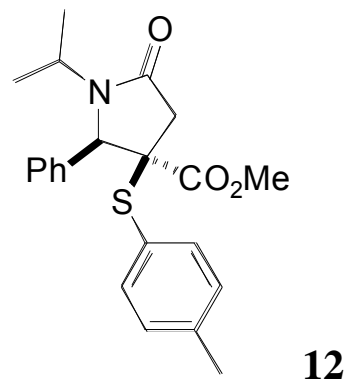

12 was prepared from maleic anhydride $(98 \mathrm{mg}, 1.0 \mathrm{mmol}), p$-thiocresol $(0.124 \mathrm{~g}, 1.0$ mmol), benzaldehyde $(0.106 \mathrm{~g}, 1.0 \mathrm{mmol})$, and isopropylamine (59 $\mathrm{mg}, 1.0 \mathrm{mmol})$. Purification on $\mathrm{SiO}_{2}$ (hexane: ethyl acetate = 5:1) gave light yellow solids $(0.36 \mathrm{~g}, 94 \%)$. ${ }^{1} \mathrm{H}$ NMR (500 MHz, $\left.\mathrm{CDCl}_{3}\right) \delta 7.36(\mathrm{~m}, 3 \mathrm{H}), 7.32(\mathrm{~m}, 2 \mathrm{H}), 7.04(\mathrm{~d}, J=8 \mathrm{~Hz}, 2 \mathrm{H}), 7.00$ (d, $J=8 \mathrm{~Hz}, 2 \mathrm{H}), 5.28(\mathrm{~s}, 1 \mathrm{H}), 4.20(\mathrm{~m}, 1 \mathrm{H}), 3.57(\mathrm{~s}, 3 \mathrm{H}), 3.08(\mathrm{~d}, J=16.5 \mathrm{~Hz}, 1 \mathrm{H}), 2.89$ (d, $J=16.5 \mathrm{~Hz}, 1 \mathrm{H}), 2.25(\mathrm{~s}, 3 \mathrm{H}), 1.08(\mathrm{~d}, J=7 \mathrm{~Hz}, 3 \mathrm{H}), 0.77(\mathrm{~d}, J=7 \mathrm{~Hz}, 3 \mathrm{H}) ;{ }^{13} \mathrm{C} \mathrm{NMR}$ $\left(125 \mathrm{MHz}, \mathrm{CDCl}_{3}\right) \delta 171.6,171.2,139.7,136.7,135.8,129.4,128.7,128.2,126.1,65.5$, 59.9, 52.8, 44.4, 40.9, 21.0, 20.7, 19.7; IR (thin film): 1730, 1693, 1409, 1249, 1233, 1211, $704 \mathrm{~cm}^{-1}$; LCMS: mass calcd for $\left(\mathrm{C}_{22} \mathrm{H}_{25} \mathrm{NO}_{3} \mathrm{~S}+\mathrm{H}\right)^{+} \mathrm{m} / \mathrm{z}$ 384.16, found $\mathrm{m} / \mathrm{z}$ $384.1222, \mathrm{R}_{\mathrm{T}}=1.73 \mathrm{~min}$. 


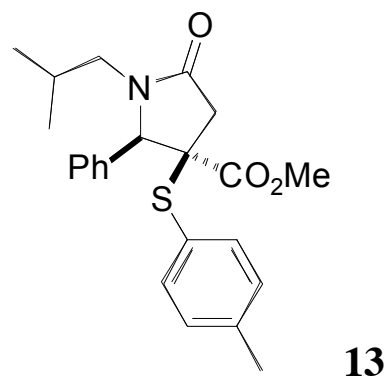

13 was prepared from maleic anhydride $(98 \mathrm{mg}, 1.0 \mathrm{mmol}), p$-thiocresol $(0.124 \mathrm{~g}, 1.0$ mmol), benzaldehyde $(0.106 \mathrm{~g}, 1.0 \mathrm{mmol})$, and isobutylamine $(73 \mathrm{mg}, 1.0 \mathrm{mmol})$. Purification on $\mathrm{SiO}_{2}$ (hexane: ethyl acetate $\left.=5: 1\right)$ gave light yellow oil $(0.36 \mathrm{~g}, 92 \%) .{ }^{1} \mathrm{H}$ NMR (500 MHz, $\left.\mathrm{CDCl}_{3}\right) \delta 7.44$ (m, 3H), 7.30 (m, 2H), 7.09 (d, $\left.J=8 \mathrm{~Hz}, 2 \mathrm{H}\right), 7.05$ (d, $J$ $=8 \mathrm{~Hz}, 2 \mathrm{H}), 5.27(\mathrm{~s}, 1 \mathrm{H}), 3.64(\mathrm{~s}, 3 \mathrm{H}), 3.54(\mathrm{dd}, J=13.5 \mathrm{~Hz}, 1 \mathrm{H}), 3.20(\mathrm{~d}, J=17 \mathrm{~Hz}$, $1 \mathrm{H}), 2.83(\mathrm{~d}, J=17 \mathrm{~Hz}, 1 \mathrm{H}), 2.36(\mathrm{~m}, 1 \mathrm{H}), 2.31(\mathrm{~s}, 3 \mathrm{H}), 1.81(\mathrm{~m}, 1 \mathrm{H}), 0.80(\mathrm{~d}, J=6.5 \mathrm{~Hz}$, $3 \mathrm{H}), 0.76(\mathrm{~d}, J=6.5 \mathrm{~Hz}, 3 \mathrm{H}) ;{ }^{13} \mathrm{C} \mathrm{NMR}\left(125 \mathrm{MHz}, \mathrm{CDCl}_{3}\right) \delta 172.3,172.1,140.1,136.1$, 134.4, 129.7, 129.2, 128.6, 126.2, 68.2, 58.8, 53.0, 48.3, 40.7, 26.4, 21.2, 20.1, 19.6; IR (thin film): 1731, 1698, 1411, 1248, $1219 \mathrm{~cm}^{-1}$; LCMS: mass calcd for $\left(\mathrm{C}_{23} \mathrm{H}_{27} \mathrm{NO}_{3} \mathrm{~S}+\mathrm{H}\right)^{+}$ $m / z 398.17$, found $m / z 398.1578, \mathrm{R}_{\mathrm{T}}=1.77 \mathrm{~min}$.

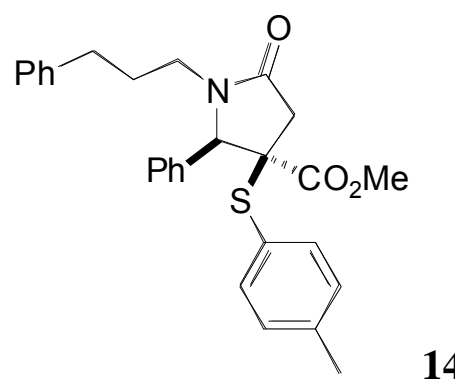

14 was prepared from maleic anhydride $(98 \mathrm{mg}, 1.0 \mathrm{mmol}), p$-thiocresol $(0.124 \mathrm{~g}, 1.0$ mmol), benzaldehyde $(0.106 \mathrm{~g}, 1.0 \mathrm{mmol})$, and 3-phenylpropylamine (0.135 g, $1.0 \mathrm{mmol})$. Purification on $\mathrm{SiO}_{2}$ (hexane: ethyl acetate $\left.=4: 1\right)$ gave light yellow oil $(0.39 \mathrm{~g}, 85 \%) .{ }^{1} \mathrm{H}$ NMR (500 MHz, $\left.\mathrm{CDCl}_{3}\right) \delta$ 7.43-7.45 (m, 3H), $7.30(\mathrm{~m}, 2 \mathrm{H}), 7.24$ (t, $\left.J=8 \mathrm{~Hz}, 2 \mathrm{H}\right), 7.16$ (t, $J=8 \mathrm{~Hz}, 1 \mathrm{H}), 7.10(\mathrm{~d}, J=8 \mathrm{~Hz}, 4 \mathrm{H}), 7.06(\mathrm{~d}, J=8 \mathrm{~Hz}, 2 \mathrm{H}), 5.30(\mathrm{~s}, 1 \mathrm{H}), 3.80(\mathrm{~m}$, 
1H), $3.63(\mathrm{~s}, 3 \mathrm{H}), 3.18(\mathrm{~d}, J=17 \mathrm{~Hz}, 1 \mathrm{H}), 2.87(\mathrm{~d}, J=17 \mathrm{~Hz}, 1 \mathrm{H}), 2.65(\mathrm{~m}, 1 \mathrm{H}), 2.48(\mathrm{t}$, $\mathrm{J}=7.5 \mathrm{~Hz}, 2 \mathrm{H}), 2.31(\mathrm{~s}, 3 \mathrm{H}), 1.72(\mathrm{~m}, 2 \mathrm{H}) ;{ }^{13} \mathrm{C} \mathrm{NMR}\left(125 \mathrm{MHz}, \mathrm{CDCl}_{3}\right) \delta 171.9,171.8$, 141.0, 139.9, 136.0, 134.2, 129.6, 129.0, 128.4, 128.2, 128.1, 126.1, 125.8, 67.5, 58.7, 52.9, 40.7, 40.5, 32.7, 28.6, 21.1; IR (thin film): 1729, 1695, 1411, 1246, 1215, $699 \mathrm{~cm}^{-1}$; LCMS: mass calcd for $\left(\mathrm{C}_{28} \mathrm{H}_{29} \mathrm{NO}_{3} \mathrm{~S}+\mathrm{H}\right)^{+} \mathrm{m} / z$ 460.19, found $\mathrm{m} / z$ 460.1115, $\mathrm{R}_{\mathrm{T}}=1.94 \mathrm{~min}$.

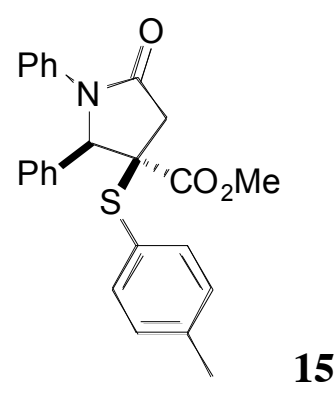

15 was prepared from maleic anhydride $(98 \mathrm{mg}, 1.0 \mathrm{mmol}), p$-thiocresol $(0.124 \mathrm{~g}, 1.0$ mmol), benzaldehyde $(0.106 \mathrm{~g}, 1.0 \mathrm{mmol})$, and aniline $(93 \mathrm{mg}, 1.0 \mathrm{mmol})$. Purification on $\mathrm{SiO}_{2}$ (hexane: ethyl acetate $\left.=4: 1\right)$ gave white solids $(0.37 \mathrm{~g}, 90 \%) .{ }^{1} \mathrm{H}$ NMR $(500 \mathrm{MHz}$, $\left.\mathrm{CDCl}_{3}\right) \delta$ 7.39-7.42 (m, 7H), 7.24 (t, $\left.J=8 \mathrm{~Hz}, 2 \mathrm{H}\right), 7.12(\mathrm{~d}, J=8 \mathrm{~Hz}, 2 \mathrm{H}), 7.06-7.09(\mathrm{~m}$, 3H), $5.81(\mathrm{~s}, 1 \mathrm{H}), 3.67(\mathrm{~s}, 3 \mathrm{H}), 3.31(\mathrm{~d}, J=17 \mathrm{~Hz}, 1 \mathrm{H}), 3.05(\mathrm{~d}, J=17 \mathrm{~Hz}, 1 \mathrm{H}), 2.33$ (s, $3 \mathrm{H}) ;{ }^{13} \mathrm{C}$ NMR (125 MHz, $\left.\mathrm{CDCl}_{3}\right) \delta 171.8,171.0,140.3,137.6,136.3,134.8,129.8$, $129.1,128.8,128.7,128.1,126.0,125.4,122.0,69.7,58.7,53.2,41.6,21.3$; IR (thin film): 1731, 1708, 1498, 1380, 1254, $1218 \mathrm{~cm}^{-1}$; LCMS: mass calcd for $\left(\mathrm{C}_{25} \mathrm{H}_{23} \mathrm{NO}_{3} \mathrm{~S}+\mathrm{H}\right)^{+}$ $\mathrm{m} / \mathrm{z}$ 418.14, found $\mathrm{m} / \mathrm{z} 418.0732, \mathrm{RT}=1.79 \mathrm{~min}$

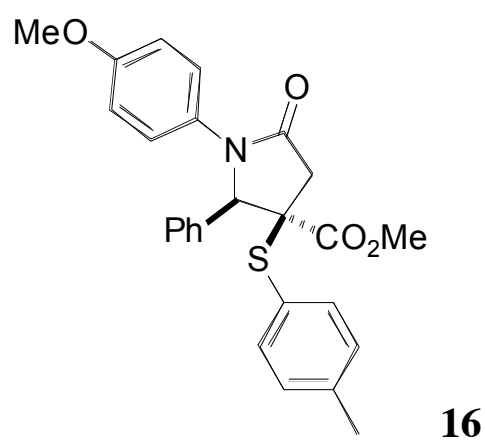


16 was prepared from maleic anhydride $(98 \mathrm{mg}, 1.0 \mathrm{mmol}), p$-thiocresol $(0.124 \mathrm{~g}, 1.0$ mmol), benzaldehyde $(0.106 \mathrm{~g}, 1.0 \mathrm{mmol})$, and 4-methoxy aniline $(0.123 \mathrm{~g}, 1.0 \mathrm{mmol})$. Purification on $\mathrm{SiO}_{2}$ (hexane: ethyl acetate $=5: 1$ ) gave light yellow oil $(0.28 \mathrm{~g}, 63 \%) .{ }^{1} \mathrm{H}$ NMR (500 MHz, CDCl $) \delta 7.41(\mathrm{~m}, 5 \mathrm{H}), 7.27$ (d, $J=9 \mathrm{~Hz}, 2 \mathrm{H}), 7.13$ (d, $J=8 \mathrm{~Hz}, 2 \mathrm{H})$, $7.08(\mathrm{~d}, J=8 \mathrm{~Hz}, 2 \mathrm{H}), 6.76(\mathrm{~d}, J=9 \mathrm{~Hz}, 2 \mathrm{H}), 5.74(\mathrm{~s}, 1 \mathrm{H}), 3.71(\mathrm{~s}, 3 \mathrm{H}), 3.67(\mathrm{~s}, 3 \mathrm{H})$, $3.30(\mathrm{~d}, J=17 \mathrm{~Hz}, 1 \mathrm{H}), 3.05$ (d, $J=17 \mathrm{~Hz}, 1 \mathrm{H}), 2.33$ (s, 3H); ${ }^{13} \mathrm{C}$ NMR $(125 \mathrm{MHz}$, $\left.\mathrm{CDCl}_{3}\right) \delta 171.8,170.8,157.2,140.2,136.2,134.9,130.5,129.7,129.0,128.6,128.2$, 126.0, 124.1, 114.0, 70.1, 58.8, 55.3, 53.1, 41.3, 21.2; IR (thin film): 1729, 1698, 1509, 1246, 1218, 1034, 729, $702 \mathrm{~cm}^{-1}$; LCMS: mass calcd for $\left(\mathrm{C}_{26} \mathrm{H}_{25} \mathrm{NO}_{4} \mathrm{~S}+\mathrm{H}\right)^{+} \mathrm{m} / z$, 448.15, found $m / z 448.1205, \mathrm{R}_{\mathrm{T}}=1.78 \mathrm{~min}$.

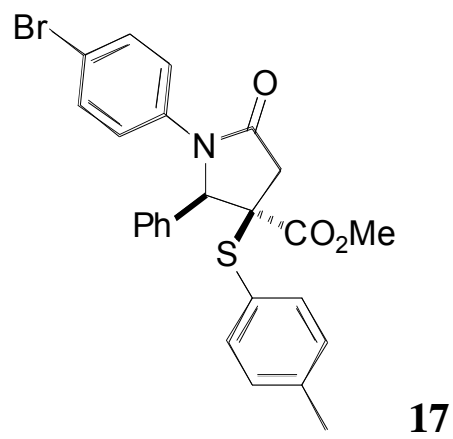

17 was prepared from maleic anhydride $(98 \mathrm{mg}, 1.0 \mathrm{mmol}), p$-thiocresol $(0.124 \mathrm{~g}, 1.0$ $\mathrm{mmol})$, benzaldehyde $(0.106 \mathrm{~g}, 1.0 \mathrm{mmol})$, and 4-bromoaniline $(0.172 \mathrm{~g}, 1.0 \mathrm{mmol})$. Purification on $\mathrm{SiO}_{2}$ (hexane: ethyl acetate $\left.=5: 1\right)$ gave light yellow oil $(0.44 \mathrm{~g}, 88 \%) .{ }^{1} \mathrm{H}$ NMR (500 MHz, CDCl $) \delta 7.39$ (m, 5H), $7.33(\mathrm{~m}, 4 \mathrm{H}), 7.12(\mathrm{~d}, J=8 \mathrm{~Hz}, 2 \mathrm{H}), 7.08$ (d, $J$ $=8 \mathrm{~Hz}, 2 \mathrm{H}), 5.79(\mathrm{~s}, 1 \mathrm{H}), 3.66(\mathrm{~s}, 3 \mathrm{H}), 3.30(\mathrm{~d}, J=17 \mathrm{~Hz}, 1 \mathrm{H}), 3.04(\mathrm{~d}, J=17 \mathrm{~Hz}, 1 \mathrm{H})$, 2.33 (s, 3H); ${ }^{13} \mathrm{C}$ NMR (125 MHz, $\left.\mathrm{CDCl}_{3}\right) \delta$ 171.6, 170.9, 140.3, 136.7, 136.3, 134.3, $131.8,129.8,129.2,128.7,128.0,125.8,123.2,118.3,69.3,58.5,53.2,41.4,21.2$; IR (thin film): 1705, 1704, 1489, 1375, 1215, 727, $699 \mathrm{~cm}^{-1}$; LCMS: mass calcd for $\left(\mathrm{C}_{22} \mathrm{H}_{22} \mathrm{BrNO}_{3} \mathrm{~S}+\mathrm{H}\right)^{+} \mathrm{m} / z 496.05$ found $m / z$ 496.0518, $\mathrm{R}_{\mathrm{T}}=1.89 \mathrm{~min}$. 


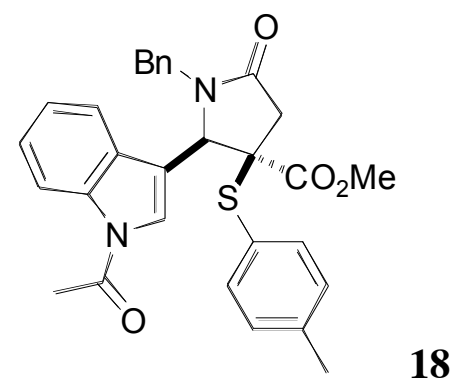

18 was prepared from maleic anhydride $(98 \mathrm{mg}, 1.0 \mathrm{mmol}), p$-thiocresol $(0.124 \mathrm{~g}, 1.0$ mmol), 1-Acetyl-3-indolecarboxaldehyde (0.187g, $1.0 \mathrm{mmol})$, and 4-bromoaniline (0.172 $\mathrm{g}, 1.0 \mathrm{mmol}$ ). Purification on $\mathrm{SiO}_{2}$ (hexane: ethyl acetate $=2: 1$ ) gave light yellow solids (0.41 g, 80\%). ${ }^{1} \mathrm{H}$ NMR (500 MHz, Toluene-D8, $\left.110{ }^{\circ} \mathrm{C}\right) \delta 8.67(\mathrm{~d}, J=8 \mathrm{~Hz}, 1 \mathrm{H}), 7.73$ (d, $J=8 \mathrm{~Hz}, 1 \mathrm{H}), 7.32(\mathrm{t}, J=7.5 \mathrm{~Hz}, 1 \mathrm{H}), 7.26$ (t, $J=7.5 \mathrm{~Hz}, 1 \mathrm{H}), 7.22(\mathrm{~s}, 1 \mathrm{H}), 7.17$ (m, 2H), $7.07(\mathrm{~m}, 5 \mathrm{H}), 6.80(\mathrm{~d}, J=8 \mathrm{~Hz}, 2 \mathrm{H}), 5.56(\mathrm{~s}, 1 \mathrm{H}), 5.10(\mathrm{~d}, J=14.5 \mathrm{~Hz}, 1 \mathrm{H}), 3.82(\mathrm{~d}$, $J=14.5 \mathrm{~Hz}, 1 \mathrm{H}), 3.44$ (d, $J=17 \mathrm{~Hz}, 1 \mathrm{H}), 3.37$ (s, 3H), 3.09 (d, $J=17 \mathrm{~Hz}, 1 \mathrm{H}), 2.08$ (s, 3H), 2.07 (s, 3H); ${ }^{13} \mathrm{C}$ NMR (125 MHz, $\left.\mathrm{CDCl}_{3}, 60{ }^{\circ} \mathrm{C}\right) \delta 171.8,171.5,168.2,140.1$, 136.1, 136.0, 129.7, 128.6, 128.3, 127.8, 126.5, 125.8, 124.0, 119.9, 116.8, 116.5, 58.8, 52.9, 52.8, 45.2, 29.7, 23.9, 21.1; IR (thin film): 1732, 1700, 1450, 1249, $1218 \mathrm{~cm}^{-1}$; LCMS: mass calcd for $\left(\mathrm{C}_{30} \mathrm{H}_{28} \mathrm{~N}_{2} \mathrm{O}_{4} \mathrm{~S}+\mathrm{H}\right)^{+} \mathrm{m} / \mathrm{z} 513.18$ found $\mathrm{m} / \mathrm{z} 513.1639, \mathrm{R}_{\mathrm{T}}=1.78 \mathrm{~min}$.

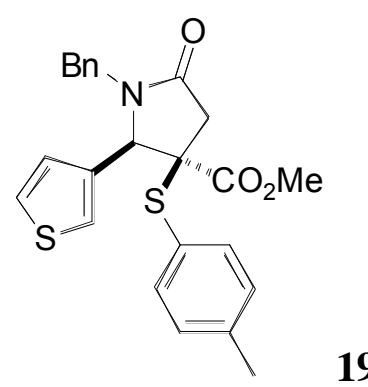

19 was prepared from maleic anhydride $(98 \mathrm{mg}, 1.0 \mathrm{mmol}), p$-thiocresol $(0.124 \mathrm{~g}, 1.0$ mmol), 3-thiophenecarboxaldedye $(0.112 \mathrm{~g}, 1.0 \mathrm{mmol})$, and benzylamine $(0.107 \mathrm{~g}, 1.0$ mmol). Purification on $\mathrm{SiO}_{2}$ (hexane: ethyl acetate $\left.=5: 1\right)$ gave light yellow oil $(0.41 \mathrm{~g}$, 93\%). ${ }^{1} \mathrm{H}$ NMR (500 MHz, $\mathrm{CDCl}_{3}$ ) $87.43(\mathrm{~m}, 1 \mathrm{H}), 7.28(\mathrm{~m}, 5 \mathrm{H}), 7.13(\mathrm{~m}, 2 \mathrm{H}), 7.08(\mathrm{~m}$, 3H), $7.03(\mathrm{~m}, 2 \mathrm{H}), 5.15(\mathrm{~d}, J=14.5 \mathrm{~Hz}, 1 \mathrm{H}), 5.07(\mathrm{~s}, 1 \mathrm{H}), 3.59(\mathrm{~d}, J=14.5 \mathrm{~Hz}, 1 \mathrm{H}), 3.54$ 
(s, 3H), $3.23(\mathrm{~d}, J=17 \mathrm{~Hz}, 1 \mathrm{H}), 2.91(\mathrm{~d}, J=17 \mathrm{~Hz}, 1 \mathrm{H}), 2.33(\mathrm{~s}, 3 \mathrm{H}) ;{ }^{13} \mathrm{C}$ NMR $(125$ $\left.\mathrm{MHz}, \mathrm{CDCl}_{3}\right) \delta 171.39,171.37,140.0,136.1,135.62,135.61,129.7,128.5,128.0,127.6$, 127.2, 126.4, 126.0, 125.3, 62.7, 58.5, 52.8, 44.5, 40.7, 21.2; IR (thin film): 1730, 1693, $1248,1215,729,700 \mathrm{~cm}^{-1}$; LCMS: mass calcd for $\left(\mathrm{C}_{24} \mathrm{H}_{23} \mathrm{NO}_{3} \mathrm{~S} \mathrm{~S}_{2}+\mathrm{H}\right)^{+} \mathrm{m} / z 438.11$ found $\mathrm{m} / \mathrm{z} 438.0923, \mathrm{R}_{\mathrm{T}}=1.78 \mathrm{~min}$.

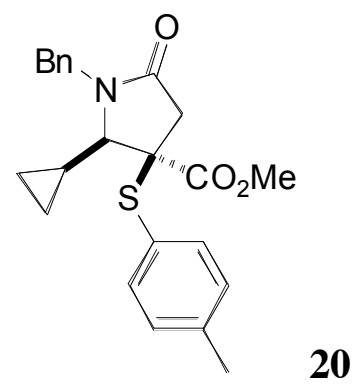

20 was prepared from maleic anhydride $(98 \mathrm{mg}, 1.0 \mathrm{mmol}), p$-thiocresol $(0.124 \mathrm{~g}, 1.0$ mmol), cyclopropanecarboxaldehyde (70 mg, $1.0 \mathrm{mmol})$, and benzylamine $(0.107 \mathrm{~g}, 1.0$ mmol). Purification on $\mathrm{SiO}_{2}$ (hexane: ethyl acetate $\left.=10: 1\right)$ gave white solids $(0.25 \mathrm{~g}$, 64\%). ${ }^{1} \mathrm{H}$ NMR (500 MHz, $\left.\mathrm{CDCl}_{3}\right) \delta$ 7.31(m, 2H), $7.27(\mathrm{~m}, 2 \mathrm{H}), 7.22(\mathrm{~m}, 1 \mathrm{H}), 5.09$ (d, $J$ $=15 \mathrm{~Hz}, 1 \mathrm{H}), 4.14(\mathrm{~d}, J=15 \mathrm{~Hz}, 1 \mathrm{H}), 3.52(\mathrm{~s}, 3 \mathrm{H}), 3.07(\mathrm{~d}, \mathrm{~J}=10 \mathrm{~Hz}, 1 \mathrm{H}), 3.06(\mathrm{~d}, J=$ $17 \mathrm{~Hz}, 1 \mathrm{H}), 2.84(\mathrm{~d}, \mathrm{~J}=17 \mathrm{~Hz}, 1 \mathrm{H}), 2.33(\mathrm{~s}, 3 \mathrm{H}), 0.98(\mathrm{~m}, 1 \mathrm{H}), 0.71(\mathrm{~m}, 2 \mathrm{H}), 0.44(\mathrm{~m}$, 1H), $0.24(\mathrm{~m}, 1 \mathrm{H}) ;{ }^{13} \mathrm{C} \mathrm{NMR}\left(125 \mathrm{MHz}, \mathrm{CDCl}_{3}\right) \delta$ 171.8, 171.0, 140.0, 136.33, 136.32, $129.8,128.5,127.38,127.37,126.4,68.2,59.4,52.6,44.2,41.2,21.2,10.7,5.1,2.8$; IR (thin film): 1729, 1692, 1251, 812, 728, $700 \mathrm{~cm}^{-1}$; LCMS: mass calcd for $\left(\mathrm{C}_{23} \mathrm{H}_{25} \mathrm{NO}_{3} \mathrm{~S}+\mathrm{H}\right)^{+} \mathrm{m} / \mathrm{z} 396.16$ found $\mathrm{m} / \mathrm{z} 396.1450, \mathrm{R}_{\mathrm{T}}=1.75 \mathrm{~min}$.

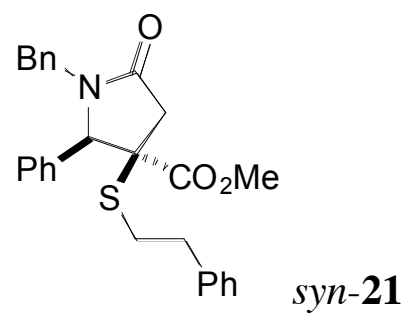


21 was prepared from maleic anhydride $(98 \mathrm{mg}, 1.0 \mathrm{mmol})$, phenyl ethanethiol $(0.138 \mathrm{~g}$, $1.0 \mathrm{mmol})$, benzaldehyde $(0.106 \mathrm{~g}, 1.0 \mathrm{mmol})$, and benzylamine $(0.107 \mathrm{~g}, 1.0 \mathrm{mmol})$. Purification on $\mathrm{SiO}_{2}$ (hexane: ethyl acetate $\left.=5: 1\right)$ gave light yellow oil $(0.24 \mathrm{~g}, 68 \%$ syn:anti $=71: 29)$.

Syn-21 ${ }^{\mathrm{H}} \mathrm{H}$ NMR (500 MHz, $\left.\mathrm{CDCl}_{3}\right) \delta 7.41(\mathrm{~m}, 3 \mathrm{H}), 7.28(\mathrm{~m}, 4 \mathrm{H}), 7.21(\mathrm{~m}, 4 \mathrm{H}), 7.18(\mathrm{t}$, $J=7.5 \mathrm{~Hz}, 1 \mathrm{H}), 7.01(\mathrm{~m}, 4 \mathrm{H}), 5.17(\mathrm{~d}, J=15 \mathrm{~Hz}, 1 \mathrm{H}), 4.97$ (s, 1H), 3.66 (s, 3H), 3.47 (d, $J=15 \mathrm{~Hz}, 1 \mathrm{H}), 3.45(\mathrm{~d}, J=17 \mathrm{~Hz}, 1 \mathrm{H}), 2.80(\mathrm{~d}, J=17 \mathrm{~Hz}, 1 \mathrm{H}), 2.46-2.59(\mathrm{~m}, 3 \mathrm{H}), 2.37-$ $2.43(\mathrm{~m}, 1 \mathrm{H}) ;{ }^{13} \mathrm{C}$ NMR $\left(125 \mathrm{MHz}, \mathrm{CDCl}_{3}\right) \delta 171.8,171.5,139.6,135.6,134.3,129.1$, 128.6, 128.4, 128.3, 128.2, 127.7, 126.4, 66.7, 55.2, 44.7, 40.7, 35.2, 31.8; IR (thin film): 1724, 1695, 1409, 1248, 1218, 729, $697 \mathrm{~cm}^{-1}$; LCMS: mass calcd for $\left(\mathrm{C}_{27} \mathrm{H}_{27} \mathrm{NO}_{3} \mathrm{~S}+\mathrm{H}\right)^{+}$ $m / z 446.17$ found $m / z 446.1025, \mathbf{R}_{\mathrm{T}}=1.86 \mathrm{~min}$.

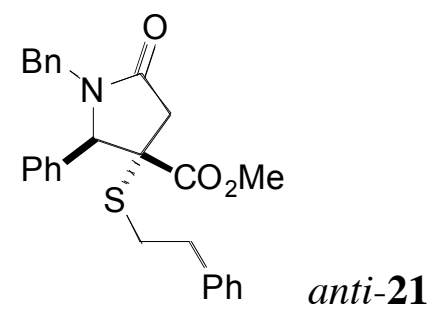

Anti-21 ${ }^{1} \mathrm{H}$ NMR (500 MHz, $\left.\mathrm{CDCl}_{3}\right) \delta$ 7.28-7.34 (m, 5H), 7.24-7.26 (m, 3H), 7.15-7.17 (m, 3H), 7.05-7.07 (m, 2H), 7.00-7.02 (m, 2H), 4.91 (d, $J=14.5 \mathrm{~Hz}, 1 \mathrm{H}), 4.76(\mathrm{~d}, J=2$ Hz, 1H), $3.82(\mathrm{~m}, 2 \mathrm{H}), 3.61(\mathrm{~m}, 2 \mathrm{H}), 3.60(\mathrm{~s}, 3 \mathrm{H}), 3.45$ (d, $J=14.5 \mathrm{~Hz}, 1 \mathrm{H}), 2.64$ (d, $J=$ $5 \mathrm{~Hz}, 2 \mathrm{H}) ;{ }^{13} \mathrm{C} \mathrm{NMR}\left(125 \mathrm{MHz}, \mathrm{CDCl}_{3}\right) \delta 171.2,137.23,137.16,136.0,129.4,129.1$, $128.9,128.5,128.44,128.41,127.5,127.3,80.1,61.8,55.8,51.5,51.0,44.0,36.5$; IR (thin film): 1737, 1702, 1435, 1168, 735, $696 \mathrm{~cm}^{-1}$; LCMS: mass calcd for $\left(\mathrm{C}_{27} \mathrm{H}_{27} \mathrm{NO}_{3} \mathrm{~S}+\mathrm{H}\right)^{+} m / z, 446.17$ found $m / z$ 445.9507, $\mathrm{R}_{\mathrm{T}}=1.86 \mathrm{~min}$. 


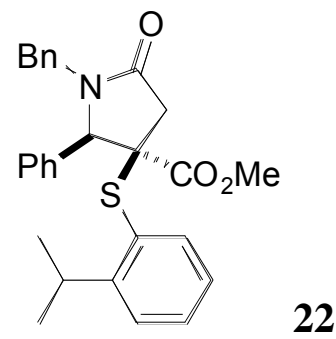

22 was prepared from maleic anhydride (98 $\mathrm{mg}, 1.0 \mathrm{mmol})$, 2-isopropylbenzenethiol (0.152 g, $1.0 \mathrm{mmol})$, benzaldehyde $(0.106 \mathrm{~g}, 1.0 \mathrm{mmol})$, and benzylamine $(0.107 \mathrm{~g}, 1.0$ mmol). Purification on $\mathrm{SiO}_{2}$ (hexane: ethyl acetate $\left.=5: 1\right)$ gave light yellow oil $(0.43 \mathrm{~g}$, 92\%). ${ }^{1} \mathrm{H}$ NMR (500 MHz, $\left.\mathrm{CDCl}_{3}\right) \delta 7.46(\mathrm{~m}, 3 \mathrm{H}), 7.27-7.31(\mathrm{~m}, 6 \mathrm{H}), 7.23-7.25(\mathrm{~m}, 1 \mathrm{H})$, 7.09-7.11 (m, 1H), 7.01-7.05 (m, 3H), $5.18(\mathrm{~d}, J=15 \mathrm{~Hz}, 1 \mathrm{H}), 5.02(\mathrm{~s}, 1 \mathrm{H}), 3.52(\mathrm{~d}, J=$ $15 \mathrm{~Hz}, 1 \mathrm{H}), 3.47(\mathrm{~s}, 3 \mathrm{H}), 3.31(\mathrm{~m}, 1 \mathrm{H}), 3.24(\mathrm{~d}, J=17 \mathrm{~Hz}, 1 \mathrm{H}), 2.83(\mathrm{~d}, J=17 \mathrm{~Hz}, 1 \mathrm{H})$, $1.09(\mathrm{t}, J=12 \mathrm{~Hz}, 6 \mathrm{H}) ;{ }^{13} \mathrm{C} \mathrm{NMR}\left(125 \mathrm{MHz}, \mathrm{CDCl}_{3}\right) \delta 171.9,171.5,153.5,137.2,135.7$, $134.1,130.2,129.1,128.58,128.57,128.3,128.1,127.7,126.0,125.9,67.2,58.7,52.8$, 44.7, 40.4, 30.4, 24.0, 23.6; IR (thin film): 1731, 1697, 1246, 1216, $700 \mathrm{~cm}^{-1}$; LCMS: mass calcd for $\left(\mathrm{C}_{28} \mathrm{H}_{29} \mathrm{NO}_{3} \mathrm{~S}+\mathrm{H}\right)^{+} \mathrm{m} / z 460.16$ found $\mathrm{m} / z$, 460.3288, $\mathrm{RT}=1.92 \mathrm{~min}$.

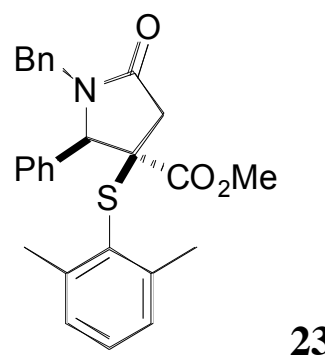

23 was prepared from maleic anhydride (98 mg, $1.0 \mathrm{mmol})$, 2, 6-dimethylthiophenol (0.138 g, $1.0 \mathrm{mmol})$, benzaldehyde $(0.106 \mathrm{~g}, 1.0 \mathrm{mmol})$, and benzylamine $(0.107 \mathrm{~g}, 1.0$ mmol). Purification on $\mathrm{SiO}_{2}$ (hexane: ethyl acetate $\left.=6: 1\right)$ gave white solids $(0.34 \mathrm{~g}, 82 \%)$. ${ }^{1} \mathrm{H}$ NMR (500 MHz, $\left.\mathrm{CDCl}_{3}\right) \delta 7.46(\mathrm{~m}, 3 \mathrm{H}), 7.34(\mathrm{~m}, 2 \mathrm{H}), 7.26(\mathrm{~m}, 3 \mathrm{H}), 7.10(\mathrm{~m}, 1 \mathrm{H})$, 6.99-7.04 (m, 4H), 5.17 (d, J = $15 \mathrm{~Hz}, 1 \mathrm{H}), 5.07$ (s, 1H), 3.51 (d, J= $15 \mathrm{~Hz}, 1 \mathrm{H}), 3.39$ (d, $J=17 \mathrm{~Hz}, 1 \mathrm{H}), 3.25(\mathrm{~s}, 3 \mathrm{H}), 2.92(\mathrm{~d}, J=17 \mathrm{~Hz}, 1 \mathrm{H}), 2.32(\mathrm{~s}, 6 \mathrm{H}) ;{ }^{13} \mathrm{C}$ NMR $(125 \mathrm{MHz}$, $\left.\mathrm{CDCl}_{3}\right) \delta 172.1,170.7,145.4,135.7,134.3,129.70,129.65,129.1,128.6,128.53 .128 .50$, 
128.1, 127.7, 67.7, 59.3, 52.5, 44.7, 41.0, 22.4; IR (thin film): 1730, 1696, 1408, 1247, 1215, 730, $700 \mathrm{~cm}^{-1}$; LCMS: mass calcd for $\left(\mathrm{C}_{27} \mathrm{H}_{27} \mathrm{NO}_{3} \mathrm{~S}+\mathrm{H}\right)^{+} \mathrm{m} / \mathrm{z} 446.17$ found $\mathrm{m} / \mathrm{z}$ 446.3143, $\mathrm{RT}=1.89 \mathrm{~min}$.

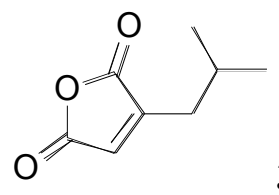

\section{3-isobutyl maleic anhydride}

3-isobutyl maleic anhydride ${ }^{2}$ was prepared from isobutyl magnesium bromide (6.3 mmol) and dimethyl acetylenedicarboxylalte (DMAD) (0.74 g, $5.2 \mathrm{mmol})$. Anhydride was achieved by vacuum evaporation of excess acetyl anhydride from 3-isobutyl maleic acid at $50{ }^{\circ} \mathrm{C}$ and quick filtration on short silicon gel column chromatography as colorless oil (0.37 g, 48\% overall). ${ }^{1} \mathrm{H}$ NMR (500 MHz, $\left.\mathrm{CDCl}_{3}\right) \delta 6.60$ (s, $\left.1 \mathrm{H}\right), 2.42(\mathrm{~d}, J=7.5 \mathrm{~Hz}$, 2H), $2.02(\mathrm{~m}, 1 \mathrm{H}), 0.99(\mathrm{~d}, J=7 \mathrm{~Hz}, 6 \mathrm{H}) ;{ }^{13} \mathrm{C} \mathrm{NMR}\left(125 \mathrm{MHz}, \mathrm{CDCl}_{3}\right) \delta 166.0,164.0$, 152.4, 129.2, 34.6, 27.1, 22.2; IR (thin film): 1842, 1762, 1243, 989, $883 \mathrm{~cm}^{-1}$.

\section{General procedure for synthesis of pentasubstituted $\gamma$-lactams by four-component}

reactions: To a solution of substituted maleic anhydride $(4.0 \mathrm{mmol}), p$-thiocresol $(0.5 \mathrm{~g}$, $4.0 \mathrm{mmol})$, and benzaldehyde $(0.42 \mathrm{~g}, 4.0 \mathrm{mmol})$ in toluene $(15 \mathrm{~mL})$ was added amine $(4.0 \mathrm{mmol})$ at room temperature. The mixture was refluxed under argon with dean stark for 24 hours. After cooled to room temperature, the solution was concentrated in vacuo. The residue was dissolved by acetone $(40 \mathrm{~mL})$, followed by addition of anhydrous $\mathrm{K}_{2} \mathrm{CO}_{3}$ $(2.2 \mathrm{~g}, 16 \mathrm{mmol})$ and $\mathrm{MeI}(2.2 \mathrm{~g}, 16 \mathrm{mmol})$ at $0{ }^{\circ} \mathrm{C}$. The mixture was warmed up to room temperature slowly and stirred overnight. The solution was concentrated in vacuo and extracted with water and ethyl acetate $(20 \mathrm{~mL} \times 3)$. The organic layers were washed with brine, dried with anhydrous $\mathrm{MgSO}_{4}$. Concentration in vacuo and purification by flash column chromatography gave final products. 


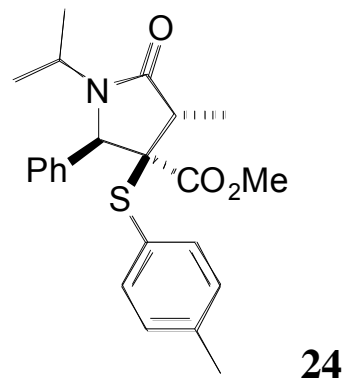

24 was prepared from citraconic anhydride $(0.45 \mathrm{~g}, 4.0 \mathrm{mmol}), p$-thiocresol $(0.5 \mathrm{~g}, 4.0$ $\mathrm{mmol})$, and benzaldehyde $(0.42 \mathrm{~g}, 4.0 \mathrm{mmol})$, and isopropylamine $(0.24 \mathrm{~g}, 4.0 \mathrm{mmol})$. Purification on $\mathrm{SiO}_{2}$ (hexane: ethyl acetate $=5: 1$ ) gave white solids $(1.12 \mathrm{~g}, 70 \%) .{ }^{1} \mathrm{H}$ NMR (500 MHz, $\left.\mathrm{CDCl}_{3}\right) \delta$ 7.30-7.35 (m, 3H), 7.25-7.27 (m, 2H), 6.99 (d, $\left.J=8 \mathrm{~Hz}, 2 \mathrm{H}\right)$, $6.96(\mathrm{~d}, J=8 \mathrm{~Hz}, 2 \mathrm{H}), 5.23(\mathrm{~s}, 1 \mathrm{H}), 4.07(\mathrm{~m}, 1 \mathrm{H}), 3.68(\mathrm{~s}, 3 \mathrm{H}), 3.02(\mathrm{dd}, J=14.5,7 \mathrm{~Hz}$, 1H), $2.30(\mathrm{~s}, 3 \mathrm{H}), 1.25(\mathrm{~d}, J=12 \mathrm{~Hz}, 3 \mathrm{H}), 1.16(\mathrm{~d}, J=7 \mathrm{~Hz}, 3 \mathrm{H}), 0.87(\mathrm{~d}, J=12 \mathrm{~Hz}$, $3 \mathrm{H}) ;{ }^{13} \mathrm{C} \mathrm{NMR}\left(125 \mathrm{MHz}, \mathrm{CDCl}_{3}\right) \delta 173.3,171.2,138.8,137.2,134.6,129.3,128.6$, $128.2,128.0,127.6,65.8,64.4,52.4,45.5,45.3,21.1,20.5,19.6,11.6$; IR (thin film): 1727, 1693, $1223 \mathrm{~cm}^{-1}$; LCMS: mass calcd for $\left(\mathrm{C}_{23} \mathrm{H}_{27} \mathrm{NO}_{3} \mathrm{~S}+\mathrm{H}\right)^{+} \mathrm{m} / z 398.17$ found $\mathrm{m} / z$ $398.1533, \mathrm{RT}=1.78 \mathrm{~min}$.

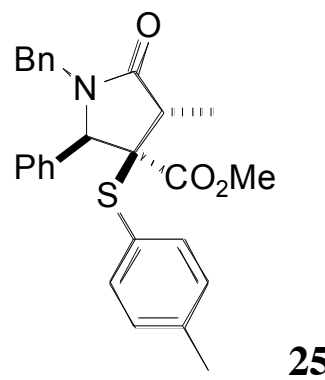

25 was prepared from citraconic anhydride $(0.45 \mathrm{~g}, 4.0 \mathrm{mmol}), p$-thiocresol $(0.5 \mathrm{~g}, 4.0$ $\mathrm{mmol})$, and benzaldehyde (0.42 $\mathrm{g}, 4.0 \mathrm{mmol})$, and benzylamine (0.43 g, $4.0 \mathrm{mmol})$. Purification on $\mathrm{SiO}_{2}$ (hexane: ethyl acetate $=6: 1$ ) gave white solids $(1.16 \mathrm{~g}, 65 \%)$. ${ }^{1} \mathrm{H}$ NMR (500 MHz, $\left.\mathrm{CDCl}_{3}\right) \delta$ 7.37-7.40 (m, 3H), 7.33-7.35 (m, 2H), 7.23-7.25 (m, 3H), $7.00(\mathrm{~m}, 4 \mathrm{H}), 6.97-6.99(\mathrm{~m}, 2 \mathrm{H}), 5.23(\mathrm{~d}, J=14.5 \mathrm{~Hz}, 1 \mathrm{H}), 4.92(\mathrm{~s}, 1 \mathrm{H}), 3.58(\mathrm{~d}, J=14.5$ $\mathrm{Hz}, 1 \mathrm{H}), 3.54(\mathrm{~s}, 3 \mathrm{H}), 2.93(\mathrm{dd}, J=14.5,7.5 \mathrm{~Hz}, 1 \mathrm{H}), 2.30(\mathrm{~s}, 3 \mathrm{H}), 1.10(\mathrm{~d}, J=7.5 \mathrm{~Hz}$, 
$3 \mathrm{H}) ;{ }^{13} \mathrm{C}$ NMR (125 MHz, $\left.\mathrm{CDCl}_{3}\right) \delta 174.8,170.6,139.6,136.6,135.5,133.3,130.2$, $129.3,128.8,128.4,128.0,127.7,126.6,64.8,62.5,52.0,45.2,44.8,21.2,12.4$; IR (thin film): 1730, 1695, 1227, 728, $700 \mathrm{~cm}^{-1}$; LCMS: mass calcd for $\left(\mathrm{C}_{27} \mathrm{H}_{27} \mathrm{NO}_{3} \mathrm{~S}+\mathrm{H}\right)^{+} \mathrm{m} / \mathrm{z}$ 446.17 found $m / z 446.1633, \mathrm{R}_{\mathrm{T}}=1.86 \mathrm{~min}$.

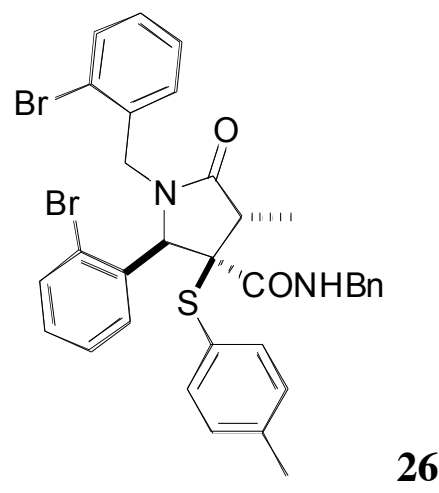

26 was prepared from citraconic anhydride $(0.45 \mathrm{~g}, 4.0 \mathrm{mmol}), p$-thiocresol $(0.5 \mathrm{~g}, 4.0$ $\mathrm{mmol})$, and 2-bromobenzaldehyde $(0.74 \mathrm{~g}, 4.0 \mathrm{mmol})$, and 2-bromobenzylamine $(0.74 \mathrm{~g}$, 4.0 mmol). The crude carboxylic acid in anhydrous DMF $(20 \mathrm{~mL})$ was added diisopropylethylamine (1.4 g, $8.8 \mathrm{mmol})$, HATU (1.67 g, $4.4 \mathrm{mmol})$, and benzylamine $(0.47 \mathrm{~g}, 4.4 \mathrm{mmol})$ at $0{ }^{\circ} \mathrm{C}$. The mixture was warmed up to room temperature slowly and stirred overnight. The mixture was diluted with ethyl acetate $(60 \mathrm{~mL})$, and then washed with water and brine. The organic layer was dried with anhydrous $\mathrm{MgSO}_{4}$. Concentration in vacuo and purification by flash column chromatography (hexane: ethyl acetate $=4: 1$ ) gave 26 as white solids $(1.32 \mathrm{~g}, 49 \%) .{ }^{1} \mathrm{H} \mathrm{NMR}\left(500 \mathrm{MHz}, \mathrm{CDCl}_{3}\right) \delta 7.99$ (t, $J=6 \mathrm{~Hz}$, 1H, NH), 7.55 (d, $J=7.5 \mathrm{~Hz}, 1 \mathrm{H}), 7.46(\mathrm{~d}, J=8 \mathrm{~Hz}, 1 \mathrm{H}), 7.40(\mathrm{~d}, J=8 \mathrm{~Hz}, 1 \mathrm{H}), 7.24-$ $7.30(\mathrm{~m}, 7 \mathrm{H}), 7.16(\mathrm{t}, J=7.5 \mathrm{~Hz}, 1 \mathrm{H}), 7.10(\mathrm{~d}, J=8 \mathrm{~Hz}, 1 \mathrm{H}), 7.06(\mathrm{t}, J=8 \mathrm{~Hz}, 1 \mathrm{H}), 6.92$ (d, $J=8 \mathrm{~Hz}, 2 \mathrm{H}), 6.88(\mathrm{~d}, J=8 \mathrm{~Hz}, 2 \mathrm{H}), 5.37(\mathrm{~s}, 1 \mathrm{H}), 4.97$ (d, $J=15 \mathrm{~Hz}, 1 \mathrm{H}), 4.60$ (dd, $J$ $=14.5,6 \mathrm{~Hz}, 1 \mathrm{H}), 4.46(\mathrm{dd}, J=14.5,5.5 \mathrm{~Hz}, 1 \mathrm{H}), 4.10(\mathrm{~d}, J=15 \mathrm{~Hz}, 1 \mathrm{H}), 2.98(\mathrm{q}, J=7$ $\mathrm{Hz}, 1 \mathrm{H}), 2.27$ (s, 3H), 0.98 (d, $J=7 \mathrm{~Hz}, 3 \mathrm{H}) ;{ }^{13} \mathrm{C} \mathrm{NMR}\left(125 \mathrm{MHz}, \mathrm{CDCl}_{3}\right) \delta 174.3,169.6$, 139.7, 137.6, 135.4, 135.0, 134.3, 132.8, 132.2, 130.8, 129.8, 129.6, 129.1, 128.9, 128.6, 128.0, 127.6, 127.5, 127.3, 126.0, 125.4, 123.7, 67.1, 63.5, 45.5, 45.1, 44.1, 21.0, 11.4; 
IR (thin film):3368 (br), 1696, 1660, 1506, 1424, 1027, 753, 732, $699 \mathrm{~cm}^{-1}$; LCMS: mass calcd for $\left(\mathrm{C}_{33} \mathrm{H}_{30} \mathrm{Br}_{2} \mathrm{~N}_{2} \mathrm{O}_{2} \mathrm{~S}+\mathrm{H}\right)^{+} \mathrm{m} / z 679.04$ found $m / z$ 678.9686, $\mathrm{R}_{\mathrm{T}}=2.02 \mathrm{~min}$.

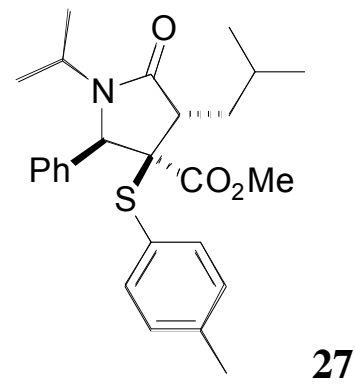

27 was prepared from 3-isobutyl maleic anhydride $(0.37 \mathrm{~g}, 2.4 \mathrm{mmol}), p$-thiocresol $(0.3 \mathrm{~g}$, $2.4 \mathrm{mmol})$, and benzaldehyde $(0.25 \mathrm{~g}, 2.4 \mathrm{mmol})$, and isopropylamine $(0.14 \mathrm{~g}, 2.4 \mathrm{mmol})$. Purification on $\mathrm{SiO}_{2}$ (hexane: ethyl acetate $\left.=10: 1\right)$ gave white solids $(0.72 \mathrm{~g}, 68 \%) .{ }^{1} \mathrm{H}$ NMR (500 MHz, $\left.\mathrm{CDCl}_{3}\right) \delta 7.32(\mathrm{~m}, 3 \mathrm{H}), 7.23(\mathrm{~m}, 2 \mathrm{H}), 6.98(\mathrm{~d}, J=8 \mathrm{~Hz}, 2 \mathrm{H}), 6.92(\mathrm{~d}, J$ $=8 \mathrm{~Hz}, 2 \mathrm{H}), 5.23(\mathrm{~s}, 1 \mathrm{H}), 4.05(\mathrm{~m}, 1 \mathrm{H}), 3.66(\mathrm{~s}, 3 \mathrm{H}), 2.98(\mathrm{dd}, J=8,5 \mathrm{~Hz} 1 \mathrm{H}), 2.29(\mathrm{~s}$, 3H), $2.08(\mathrm{~m}, 1 \mathrm{H}), 1.68(\mathrm{~m}, 1 \mathrm{H}), 1.31(\mathrm{~m}, 1 \mathrm{H}), 1.23(\mathrm{~d}, J=6.5 \mathrm{~Hz}, 3 \mathrm{H}), 0.93(\mathrm{~d}, J=6.5$ $\mathrm{Hz}, 3 \mathrm{H}), 0.86(\mathrm{dd}, J=7,2 \mathrm{~Hz}, 6 \mathrm{H}) ;{ }^{13} \mathrm{C}$ NMR $\left(125 \mathrm{MHz}, \mathrm{CDCl}_{3}\right) \delta 172.9,171.3,138.3$, $137.3,133.5,129.3,128.5,128.1,128.0,65.6,64.7,52.4,47.8,45.5,36.0,26.0,22.8$, 22.1, 21.1, 20.4, 19.6; IR (thin film): 1725, 1688, 1215, 730, $702 \mathrm{~cm}^{-1}$; LCMS: mass calcd for $\left(\mathrm{C}_{26} \mathrm{H}_{33} \mathrm{NO}_{3} \mathrm{~S}+\mathrm{H}\right)^{+} \mathrm{m} / z 440.22$ found $m / z 440.1915, \mathrm{R}_{\mathrm{T}}=2.00 \mathrm{~min}$.

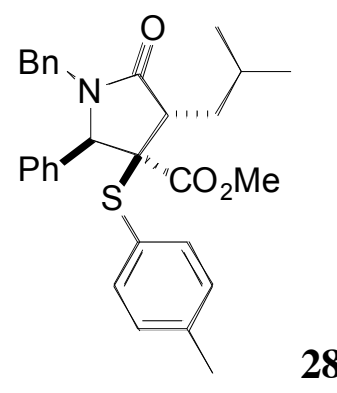

28 was prepared from 3-isobutyl maleic anhydride $(0.37 \mathrm{~g}, 2.4 \mathrm{mmol}), p$-thiocresol $(0.3 \mathrm{~g}$, $2.4 \mathrm{mmol})$, and benzaldehyde ( $0.25 \mathrm{~g}, 2.4 \mathrm{mmol})$, and benzylamine $(0.26 \mathrm{~g}, 2.4 \mathrm{mmol})$. Purification on $\mathrm{SiO}_{2}$ (hexane: ethyl acetate $\left.=10: 1\right)$ gave white solids $(0.72 \mathrm{~g}, 62 \%) .{ }^{1} \mathrm{H}$ 
NMR (500 MHz, $\left.\mathrm{CDCl}_{3}\right) \delta 7.38$ (m, 5H), $7.24(\mathrm{~m}, 3 \mathrm{H}), 6.99(\mathrm{~m}, 4 \mathrm{H}), 6.97(\mathrm{~m}, 2 \mathrm{H}), 5.22$ (d, $J=14 \mathrm{~Hz}, 1 \mathrm{H}), 4.94(\mathrm{~s}, 1 \mathrm{H}), 3.56(\mathrm{~d}, J=14 \mathrm{~Hz}, 1 \mathrm{H}), 3.52$ (s, 3H), 2.88 (dd, $J=9.5,4$ Hz, 1H), 2.29 (s, 3H), 1.99 (m, 1H), 1.45 (m, 1H), 1.07 (m, 1H), 0.84 (d, J = 6.5 Hz, 3H), $0.80(\mathrm{~d}, J=6.5 \mathrm{~Hz}, 3 \mathrm{H}) ;{ }^{13} \mathrm{C}$ NMR $\left(125 \mathrm{MHz}, \mathrm{CDCl}_{3}\right) \delta 174.1,170.5,139.2,136.1$, 135.7, 133.4, 130.4, 129.2, 128.8, 128.7, 128.4, 127.9, 127.6, 127.0, 64.7, 63.0, 52.0, 48.1, 44.7, 36.3, 25.6, 23.2, 21.5, 21.2; IR (thin film): 1731, 1690, 1230, 907, 727, $699 \mathrm{~cm}^{-1}$; LCMS: mass calcd for $\left(\mathrm{C}_{30} \mathrm{H}_{33} \mathrm{NO}_{3} \mathrm{~S}+\mathrm{H}\right)^{+} \mathrm{m} / z 488.22$ found $\mathrm{m} / z$ 488.2193, $\mathrm{R}_{\mathrm{T}}=2.05 \mathrm{~min}$.

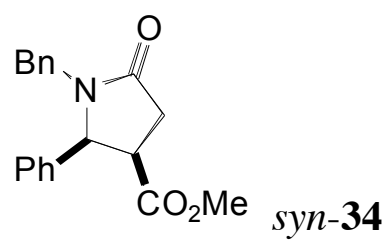

Compound $11(0.16 \mathrm{~g}, \quad 0.37 \mathrm{mmol})$, AIBN (3 $\mathrm{mg}, \quad 0.018 \mathrm{mmol})$, and tris(trimethylsilyl)silane $(0.18 \mathrm{~g}, 0.74 \mathrm{mmol})$ in toluene $(15 \mathrm{~mL})$ in sealed tube was flushed with argon and heated to $90{ }^{\circ} \mathrm{C}$ for 4 hours with stirring. The reaction was monitored by TLC till complete. After cooled to room temperature, concentration in vacuo and purification by flash column chromatography (hexane: ethyl acetate $=2: 1$ ) gave syn-34 as white solids $(0.1 \mathrm{~g}, 93 \%) .{ }^{1} \mathrm{H}$ NMR $\left(300 \mathrm{MHz}, \mathrm{CDCl}_{3}\right)$ \& 7.26-7.36 (m, 6H), 7.06-7.13 (m, 4H), $5.18(\mathrm{~d}, J=14.4 \mathrm{~Hz}, 1 \mathrm{H}), 4.69(\mathrm{~d}, J=14.4 \mathrm{~Hz}, 1 \mathrm{H}), 3.42-3.64$ (m, 2H), $3.24(\mathrm{~s}, 3 \mathrm{H}), 3.18(\mathrm{~m}, 1 \mathrm{H}), 2.63(\mathrm{~m}, 1 \mathrm{H}) ;{ }^{13} \mathrm{C} \mathrm{NMR}\left(75 \mathrm{MHz}, \mathrm{CDCl}_{3}\right)$ ठ 173.0, 170.3, 135.8, 135.5, 128.7, 128.6, 128.4, 127.7, 127.3, 62.1, 51.6, 44.5, 43.0, 31.7; IR (thin film): 1731, 1701, 1248, $702 \mathrm{~cm}^{-1}$; LCMS: mass calcd for $\left(\mathrm{C}_{19} \mathrm{H}_{19} \mathrm{NO}_{3}+\mathrm{H}\right)^{+} \mathrm{m} / \mathrm{z}$ 310.14 found $\mathrm{m} / \mathrm{z} 310.0802, \mathrm{R}_{\mathrm{T}}=1.49 \mathrm{~min}$.

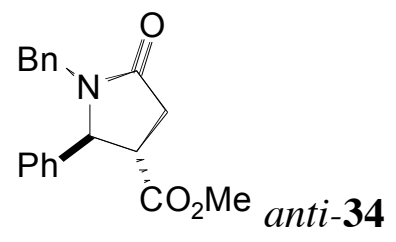


Compound 11 (0.16 g, $0.37 \mathrm{mmol})$ was dissolved in a mixture of THF and ethanol (1:2, $15 \mathrm{~mL})$. An aqueous slurry of Raney Nickel $2800(2.5 \mathrm{~mL})$ was added at room temperature under vigorous stirring. After 2 hours at room temperature, the mixture was filtered through Celite. The filtrate was extracted by brine and ethyl acetate $(15 \mathrm{~mL} \times 3)$.

The organic layers were combined and dried with anhydrous $\mathrm{MgSO}_{4}$. Concentration in vacuo and purification by flash column chromatography (hexane: ethyl acetate $=1: 1$ ) gave white solids $(0.11 \mathrm{~g}, 96 \%)$ as syn:anti (1:1) mixture. The syn, anti-34 mixture $(0.1 \mathrm{~g}$, $0.32 \mathrm{mmol})$ was dissolved in a mixture of THF and $t$-butyl alcohol (10:1, $11 \mathrm{~mL})$. Potassium $t$-butyl oxide $(11 \mathrm{mg}, 0.1 \mathrm{mmol})$ was added at room temperature and stirred over night. The solution was neutralized by $1.0 \mathrm{M}$ acetic acid, and extracted with water and ethyl acetate $(20 \mathrm{~mL} \times 3)$. The organic layers were washed with brine, dried with anhydrous $\mathrm{MgSO}_{4}$. Concentration in vacuo and purification by flash column chromatography gave anti-34 (56 mg, 56\%) as white solids. ${ }^{1} \mathrm{H} \mathrm{NMR}\left(500 \mathrm{MHz}, \mathrm{CDCl}_{3}\right)$ ठ 7.35-7.41 (m, 3H), 7.26-7.29 (m, 3H), $7.16(\mathrm{~m}, 2 \mathrm{H}), 7.04(\mathrm{~m}, 2 \mathrm{H}), 5.11(\mathrm{~d}, J=15 \mathrm{~Hz}$, $1 \mathrm{H}), 4.61(\mathrm{~d}, J=5.5 \mathrm{~Hz}, 1 \mathrm{H}), 3.65(\mathrm{~s}, 3 \mathrm{H}), 3.49(\mathrm{~d}, J=15 \mathrm{~Hz}, 1 \mathrm{H}), 3.07(\mathrm{~m}, 1 \mathrm{H}), 2.79-$ $2.92(\mathrm{~m}, 2 \mathrm{H}) ;{ }^{13} \mathrm{C} \mathrm{NMR}\left(75 \mathrm{MHz}, \mathrm{CDCl}_{3}\right) \delta 172.62,172.60,138.9,135.6,129.1,128.6$, $128.5,128.4,127.6,126.9,63.5,52.3,45.9,44.4,33.6$; IR (thin film): 1735, 1693, 1237 , $702 \mathrm{~cm}^{-1}$; LCMS: mass calcd for $\left(\mathrm{C}_{19} \mathrm{H}_{19} \mathrm{NO}_{3}+\mathrm{H}\right)^{+} \mathrm{m} / z 310.14$ found $\mathrm{m} / z$ 310.1122, $\mathrm{R}_{\mathrm{T}}=$ $1.49 \mathrm{~min}$.

\section{References:}

1. Pangborn, A. B.; Giardello, M. A.; Grubbs, R. H.; Rosen, R. K.; Timmers, F. J. Organometallics 1996, 15, 1518-1520.

2. Ratemi, E. S.; Dolence, J. M.; Poulter, C. D.; Vederas, J. C. J. Org. Chem. 1996, 61, 6296-6301. 


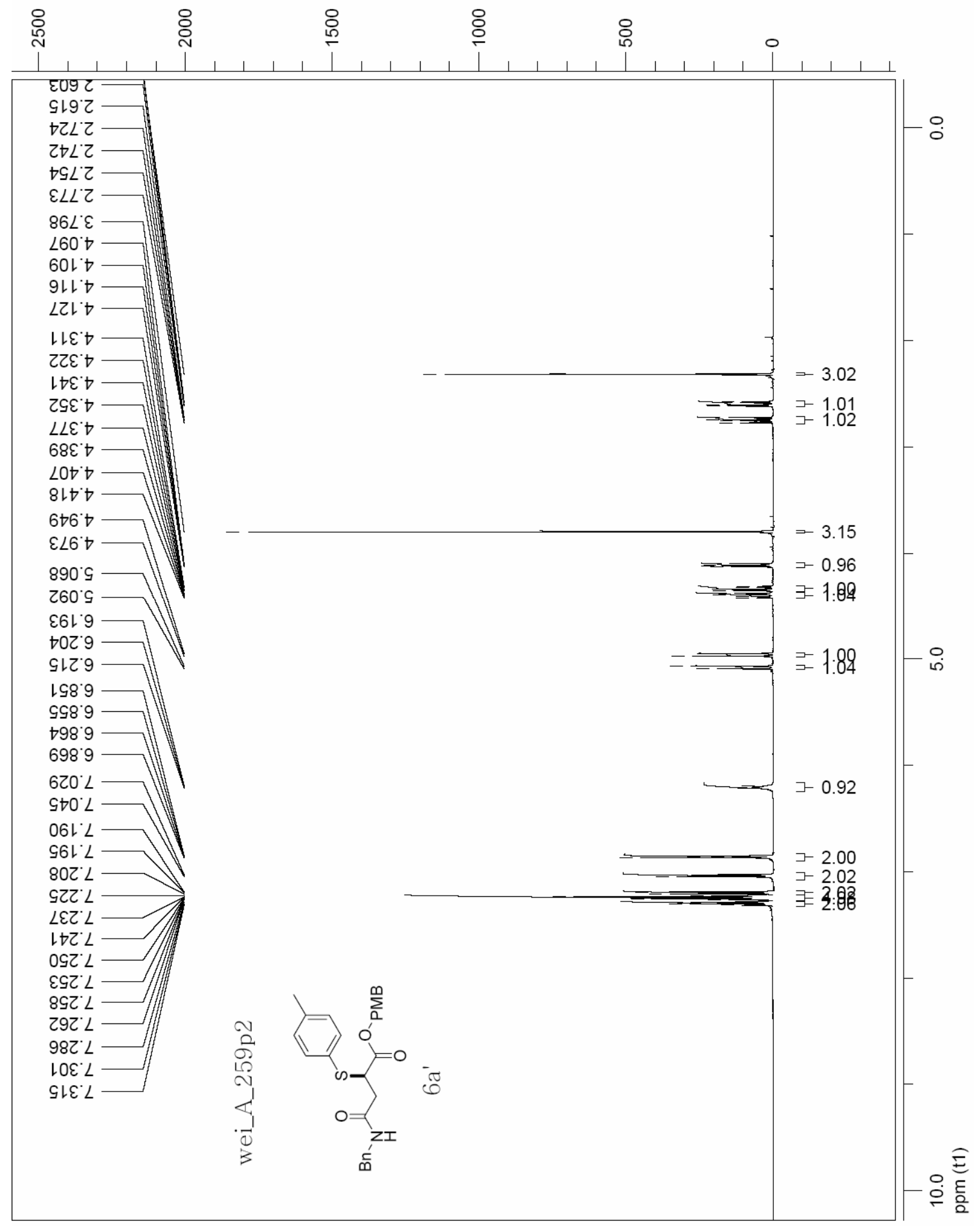




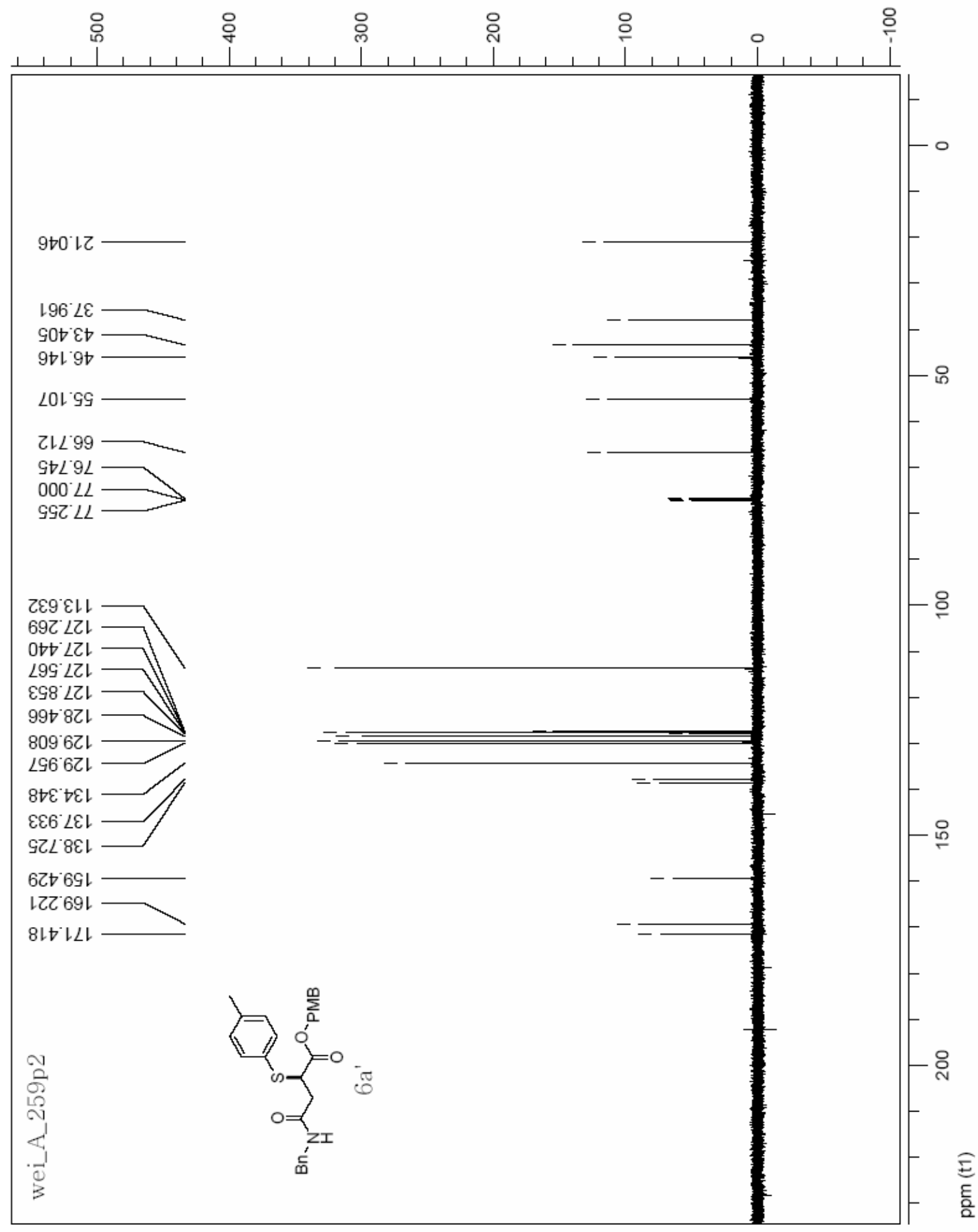




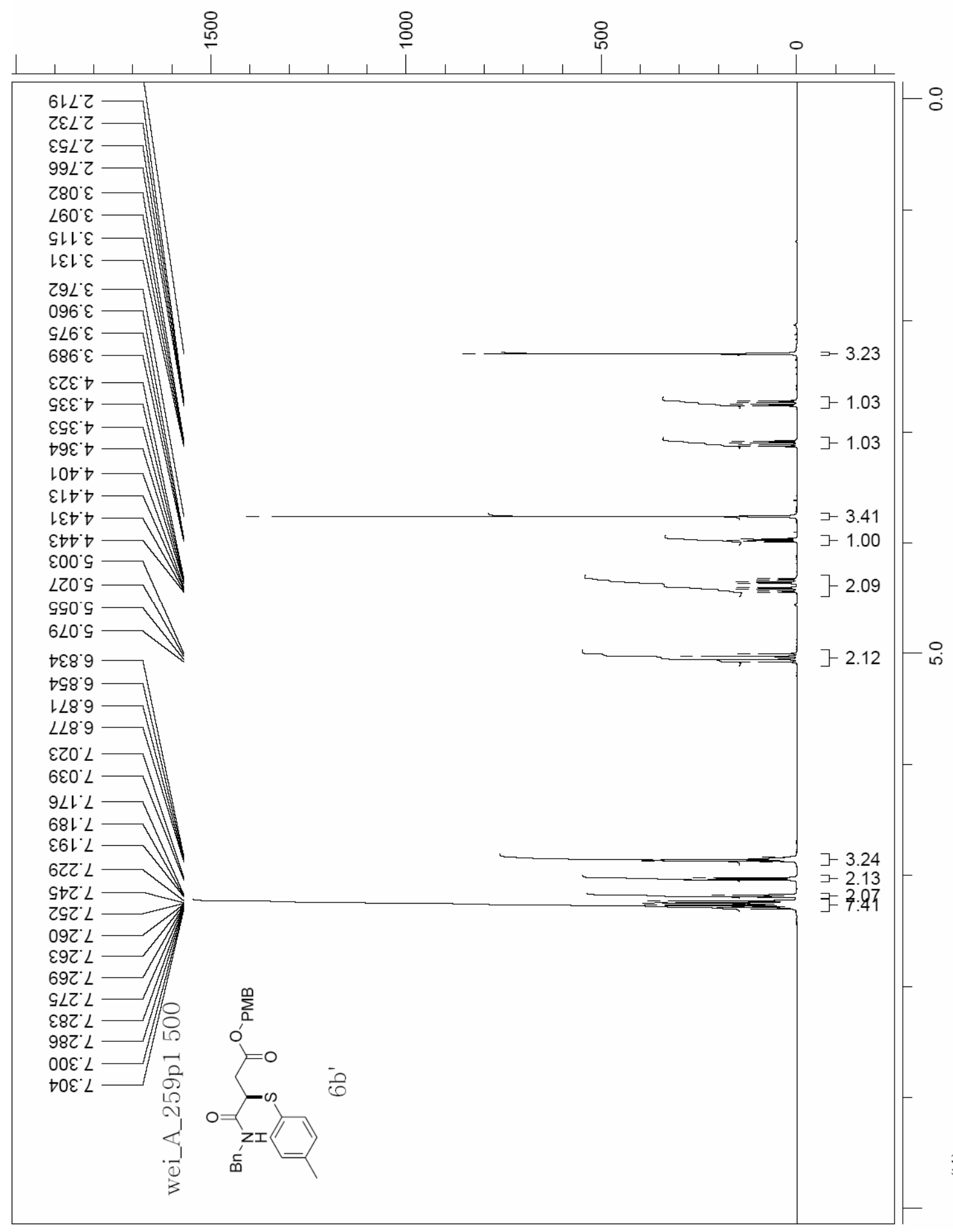

E
$\frac{0}{2}$ 


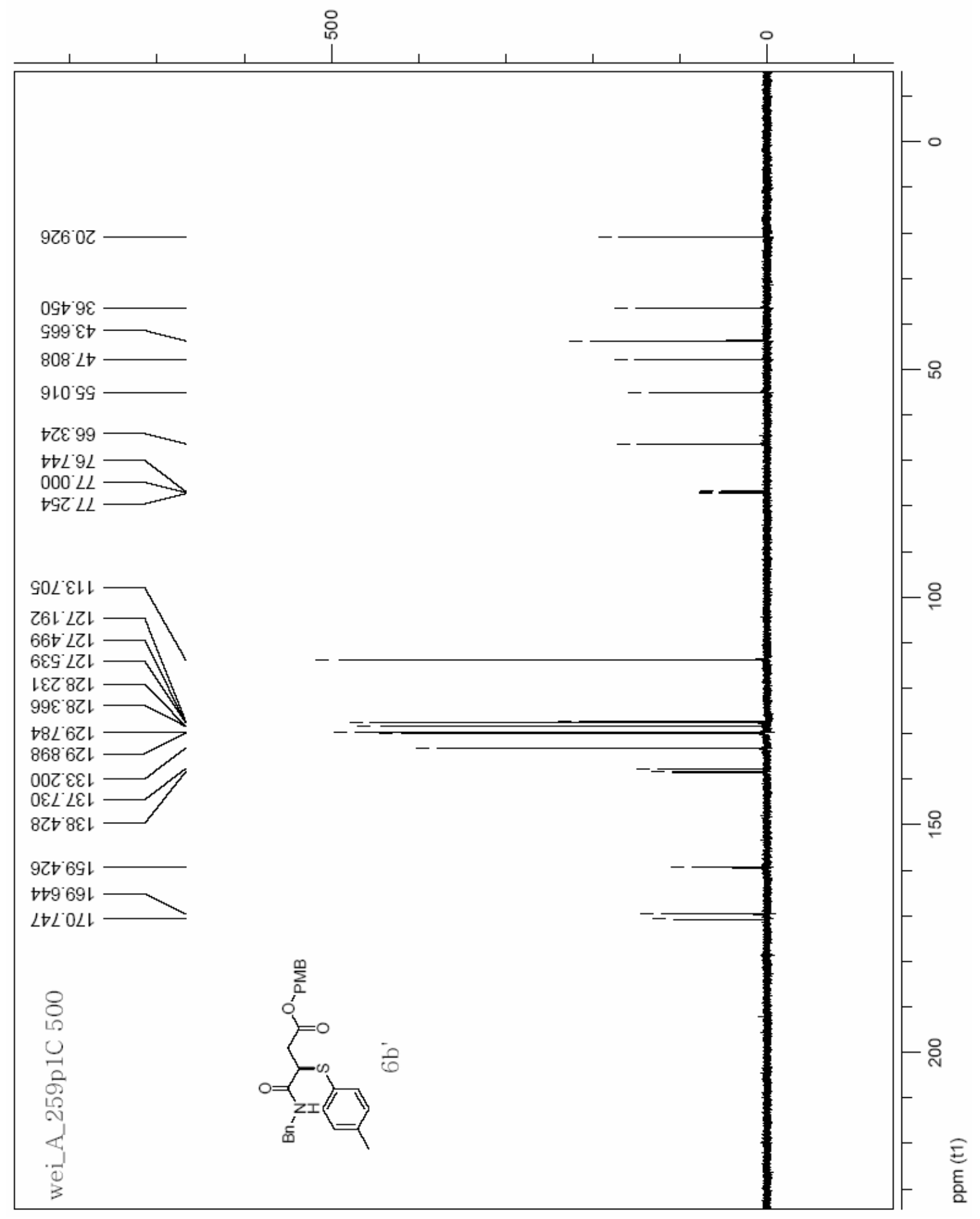




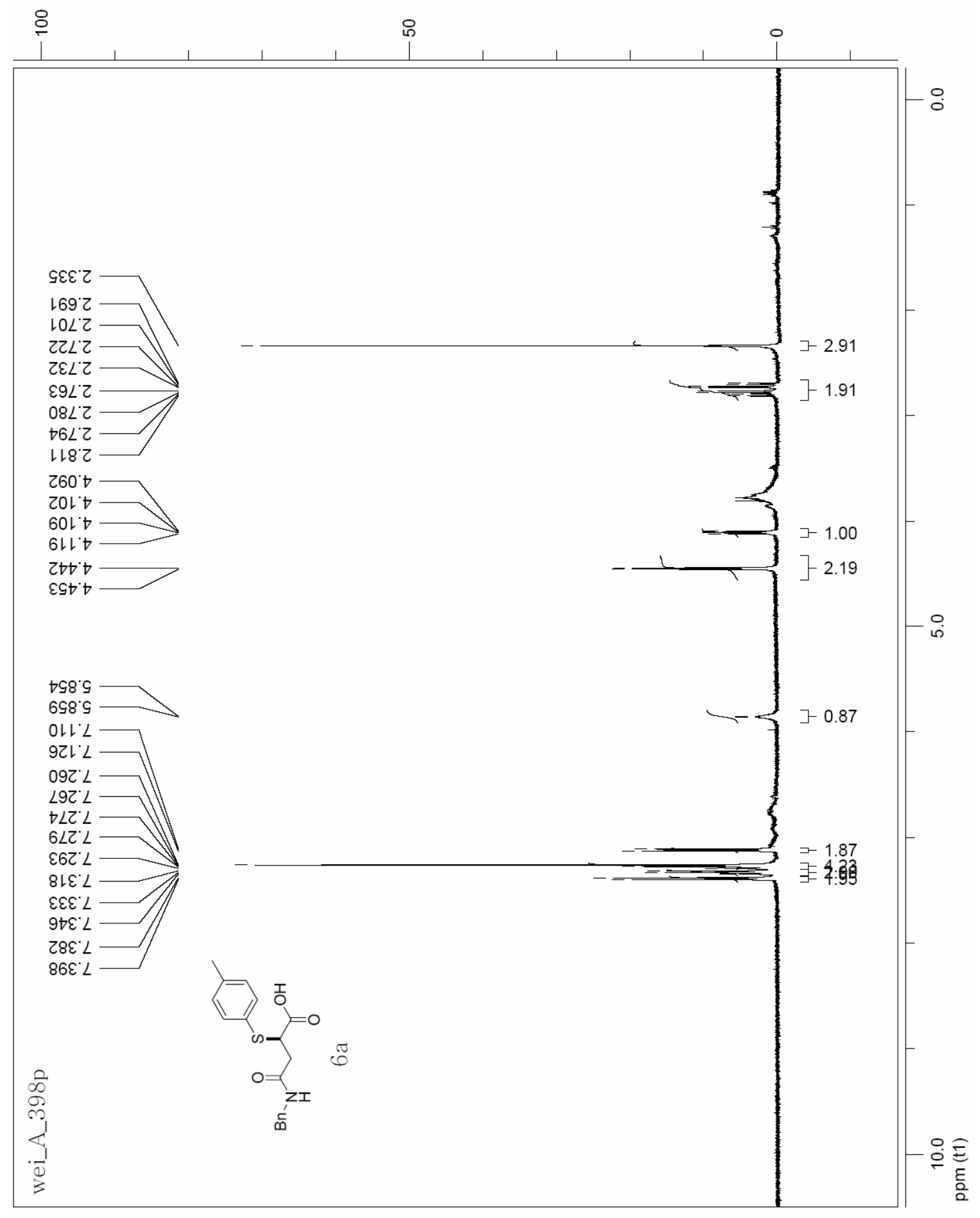




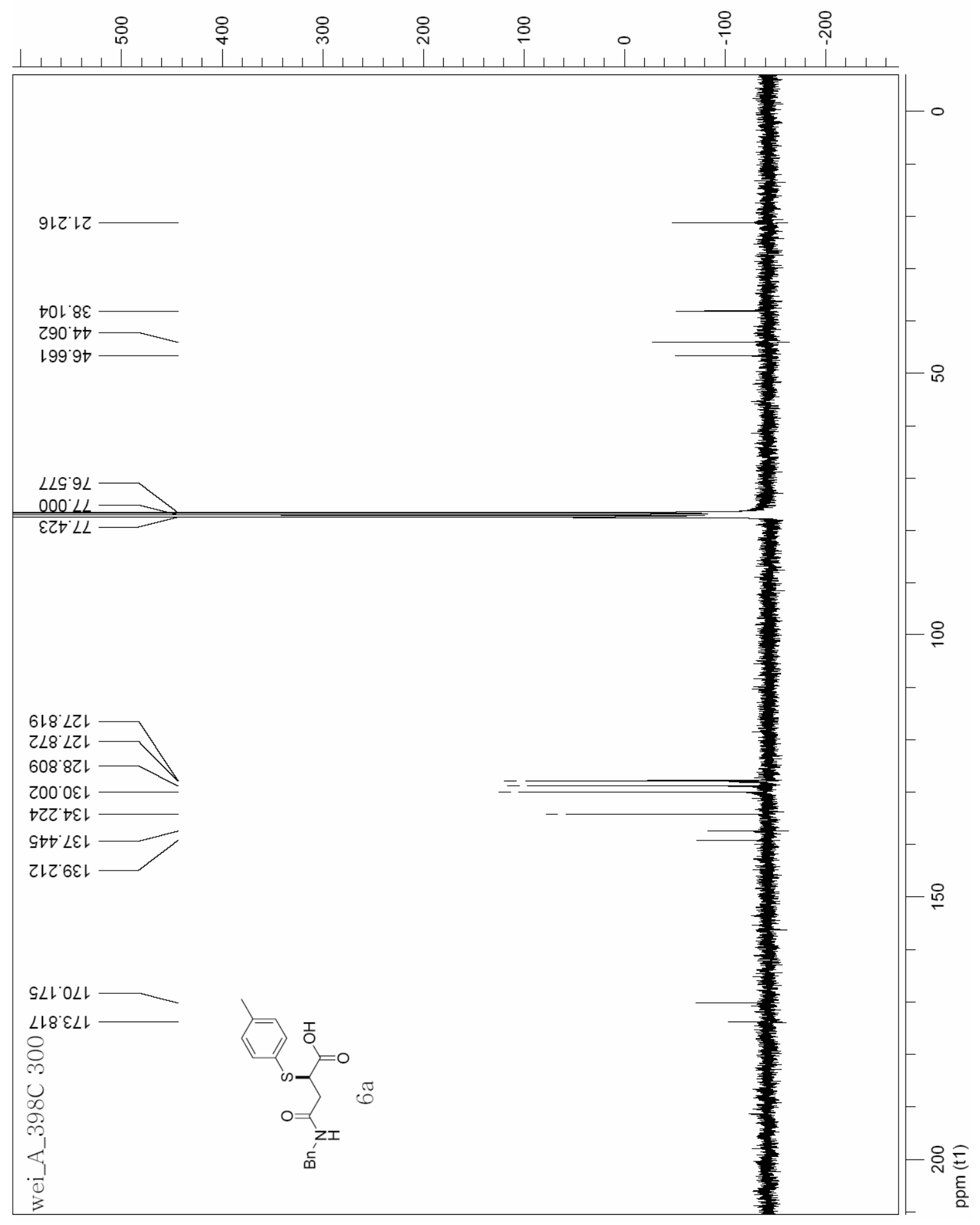




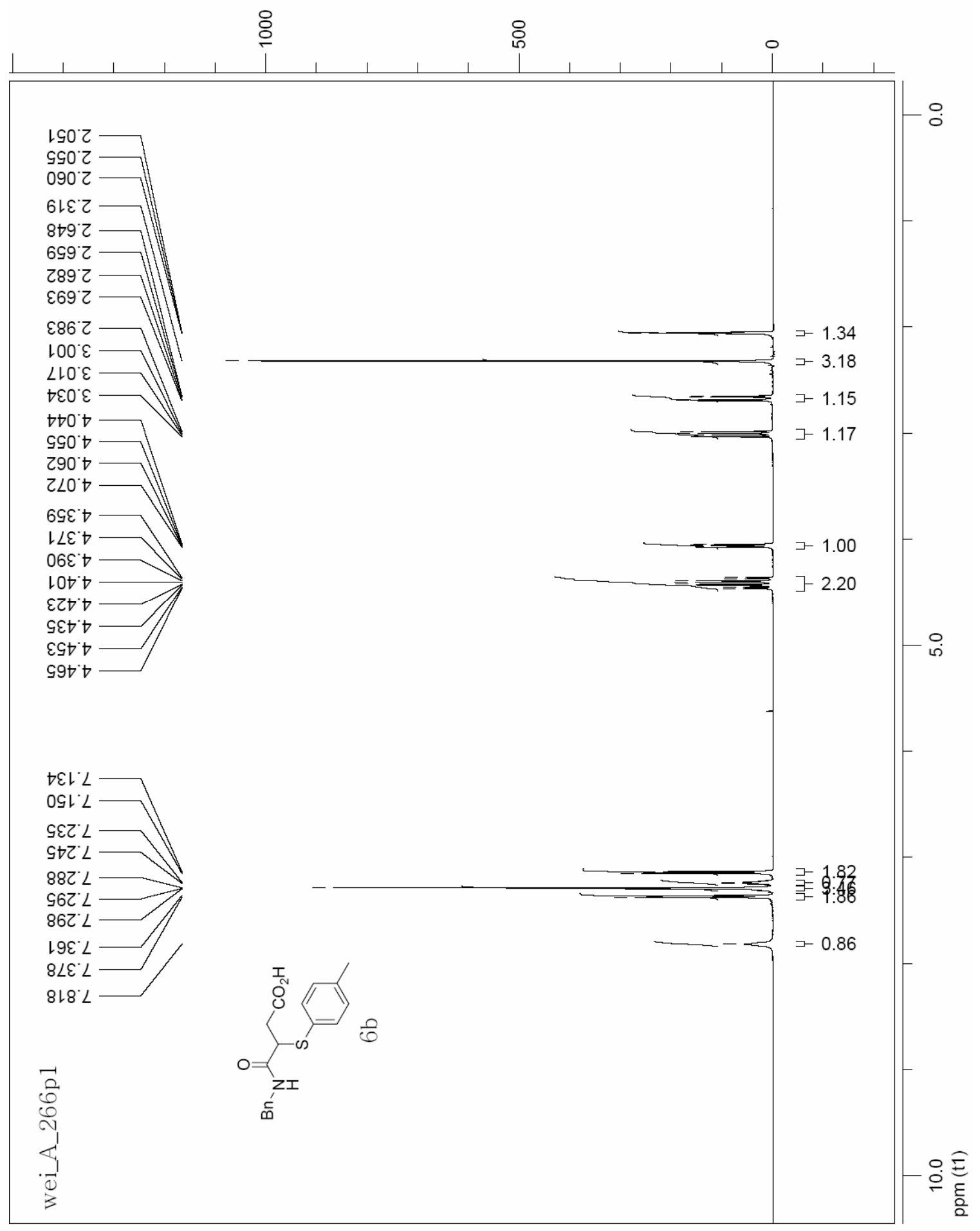




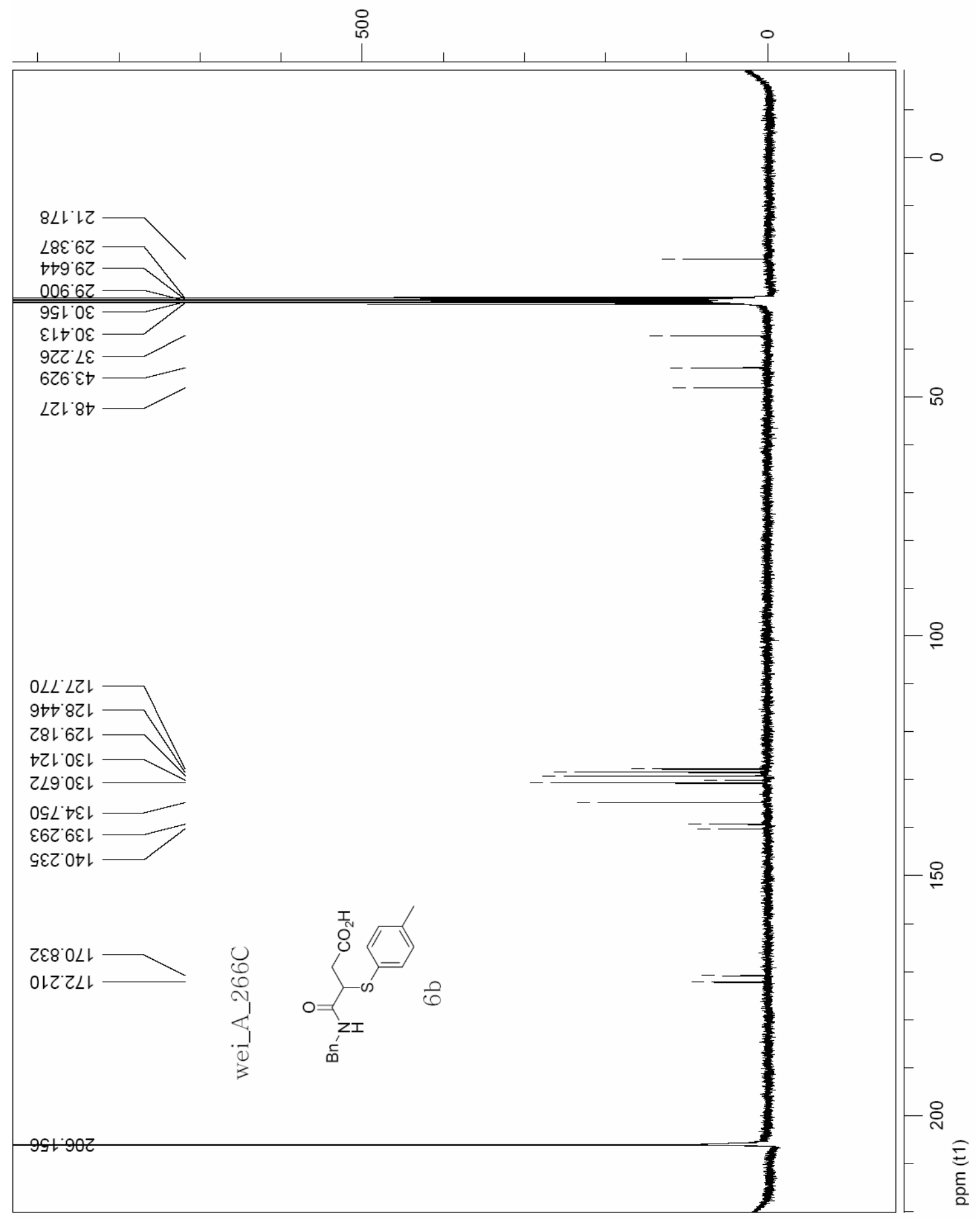




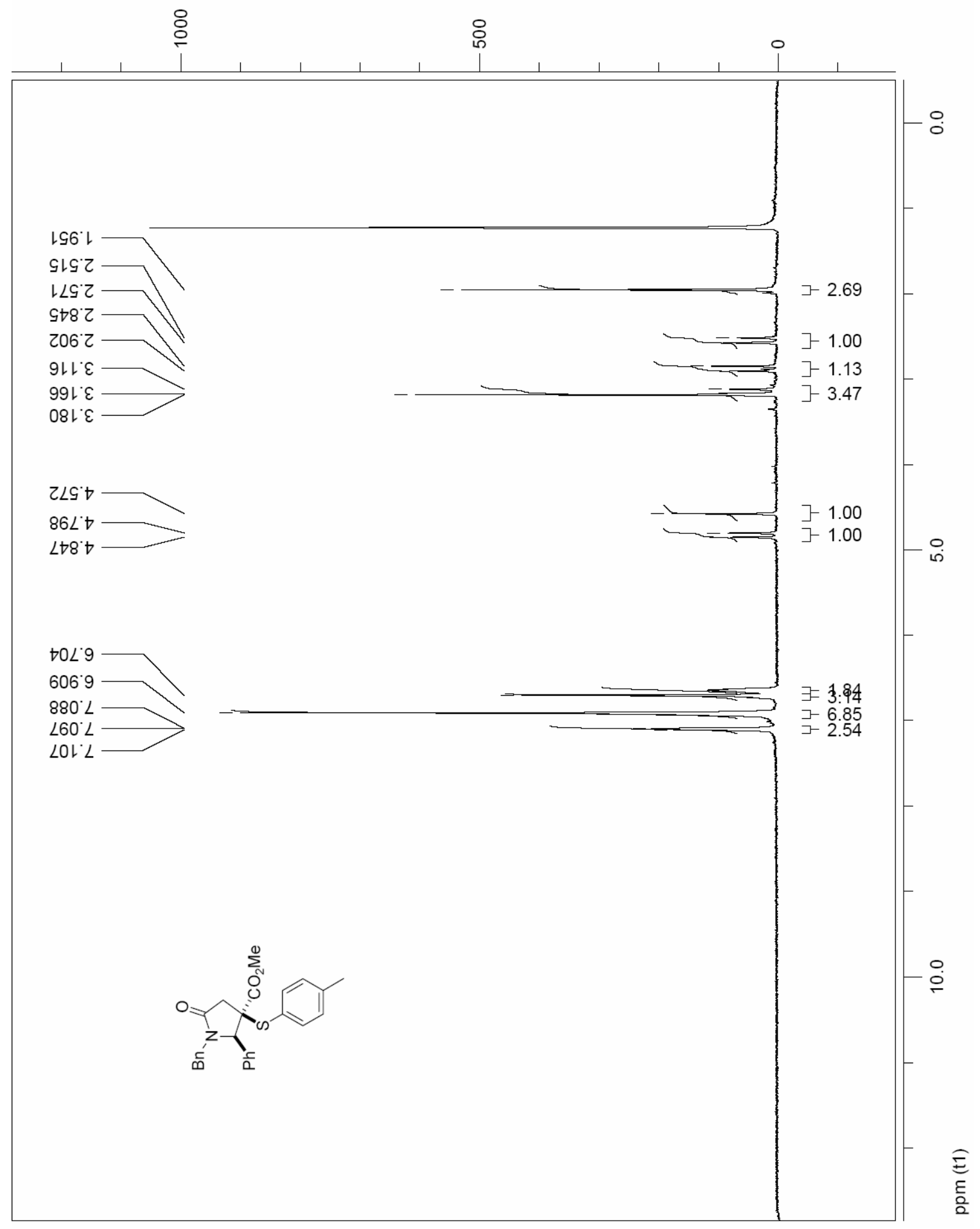




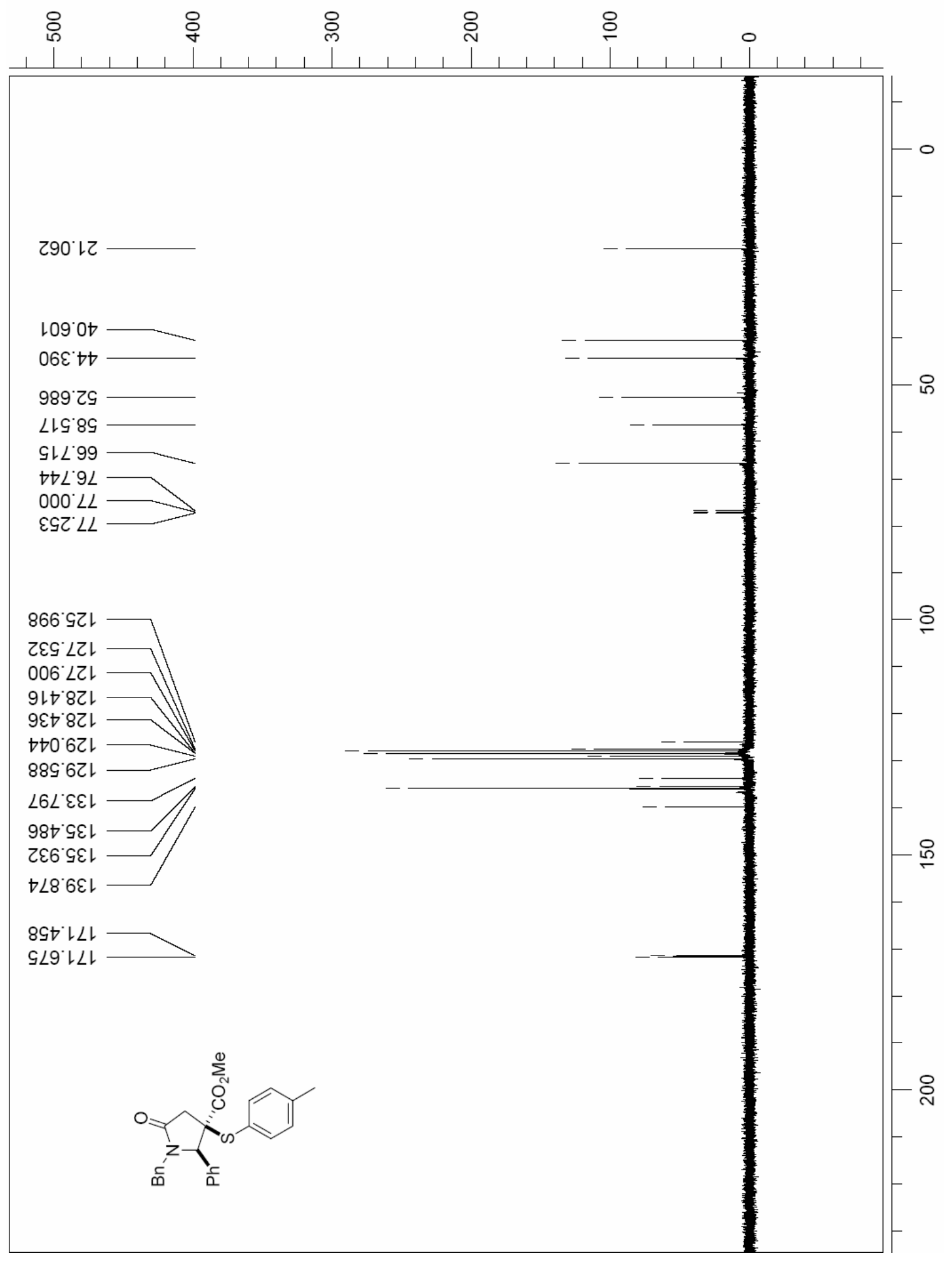




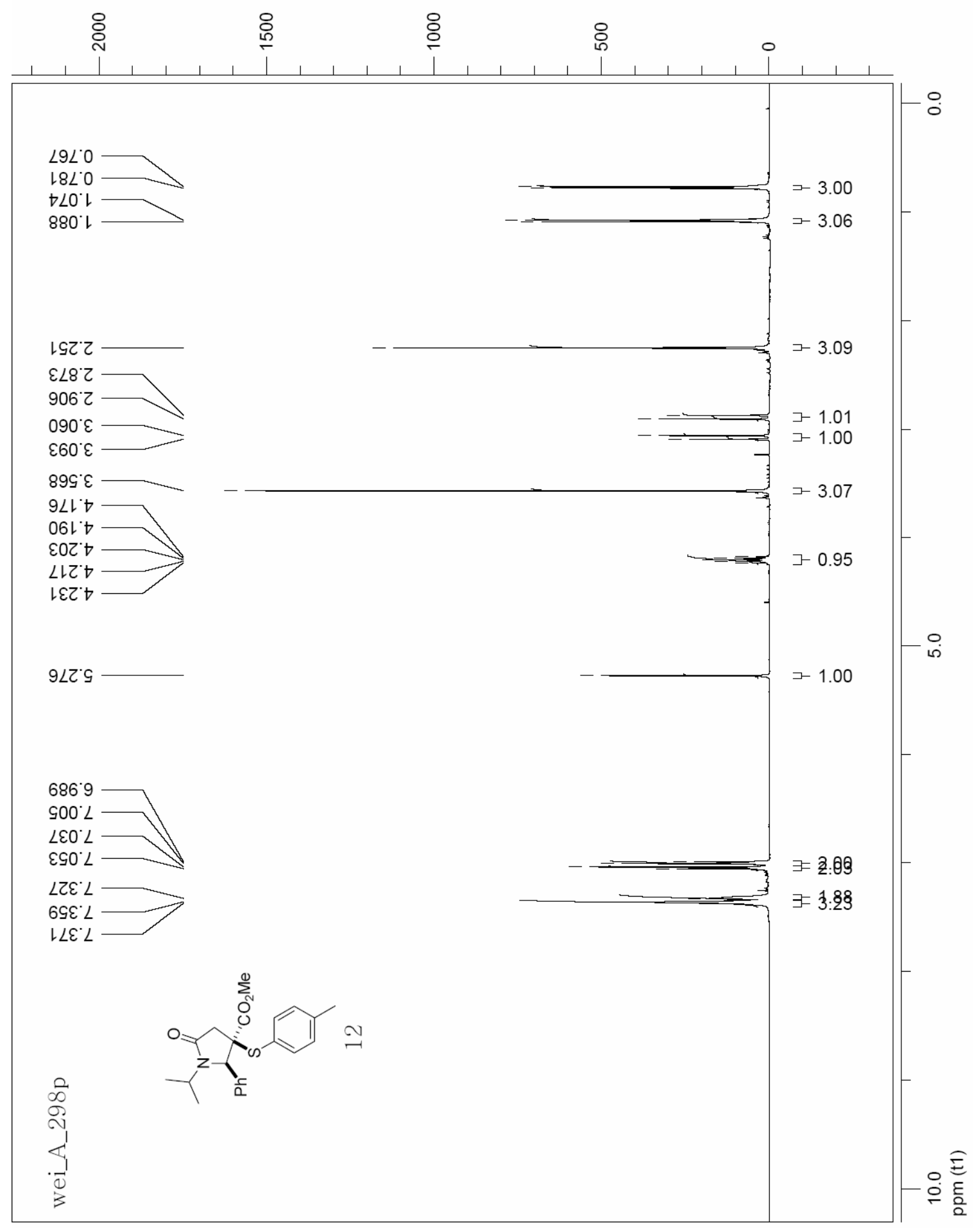




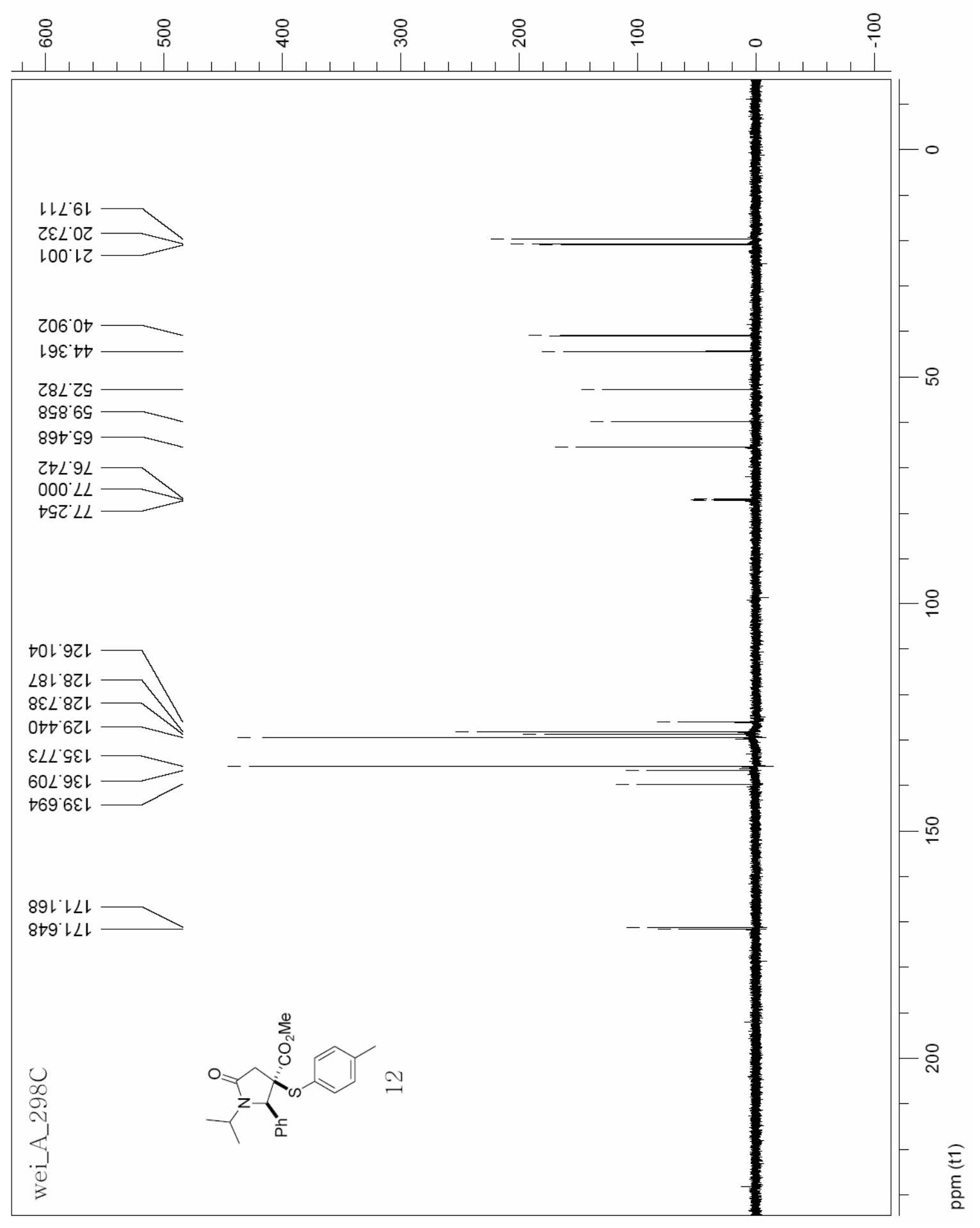




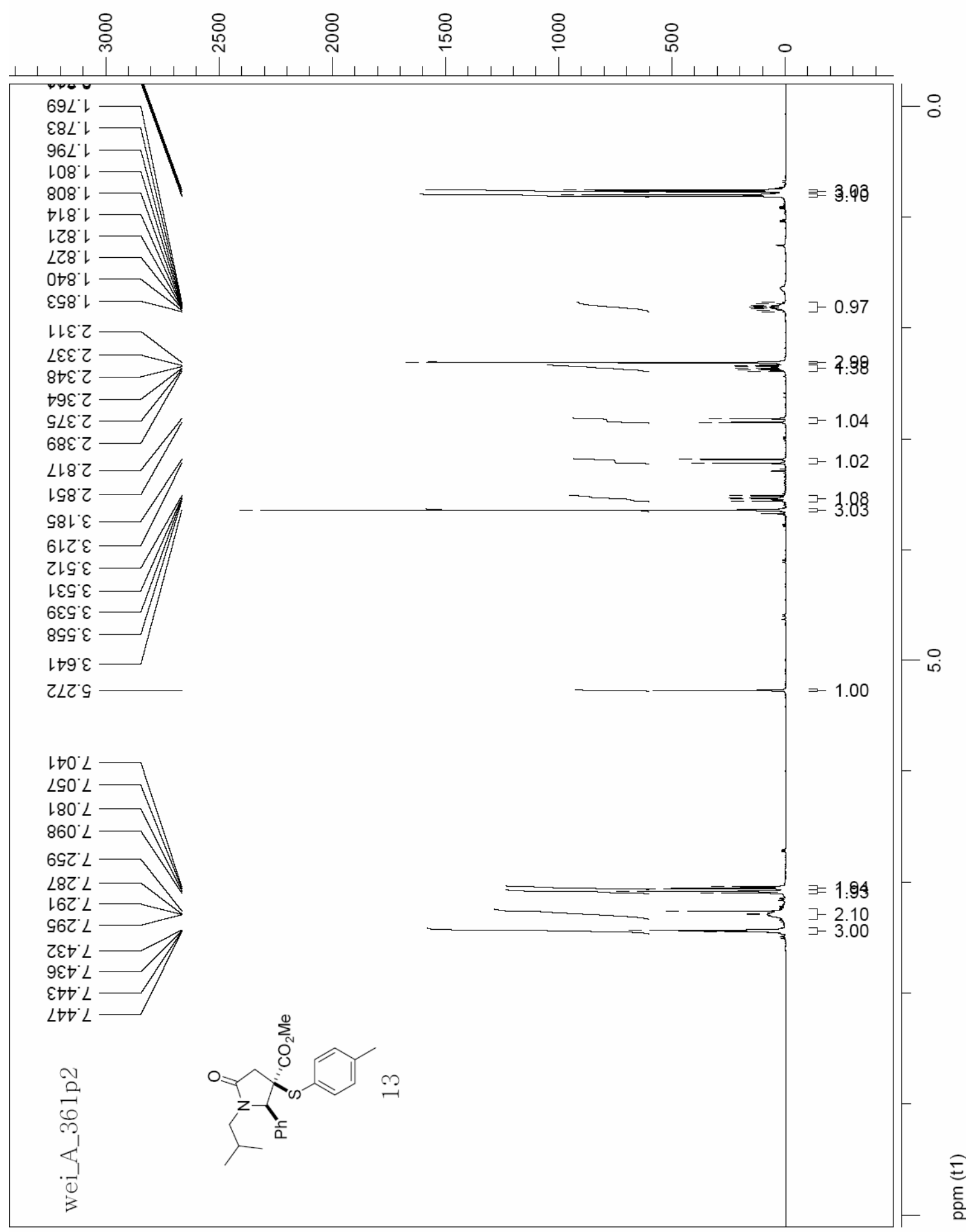




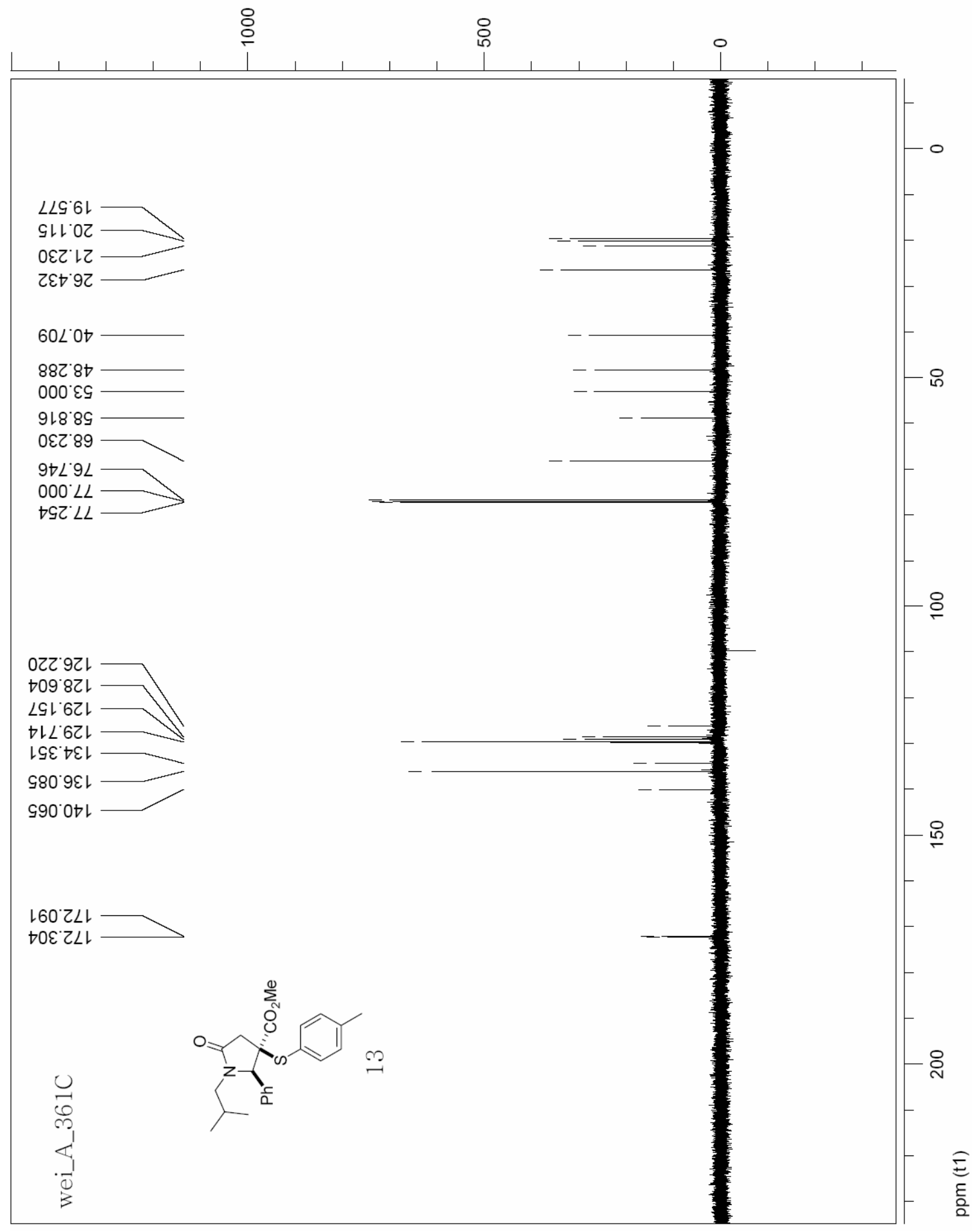




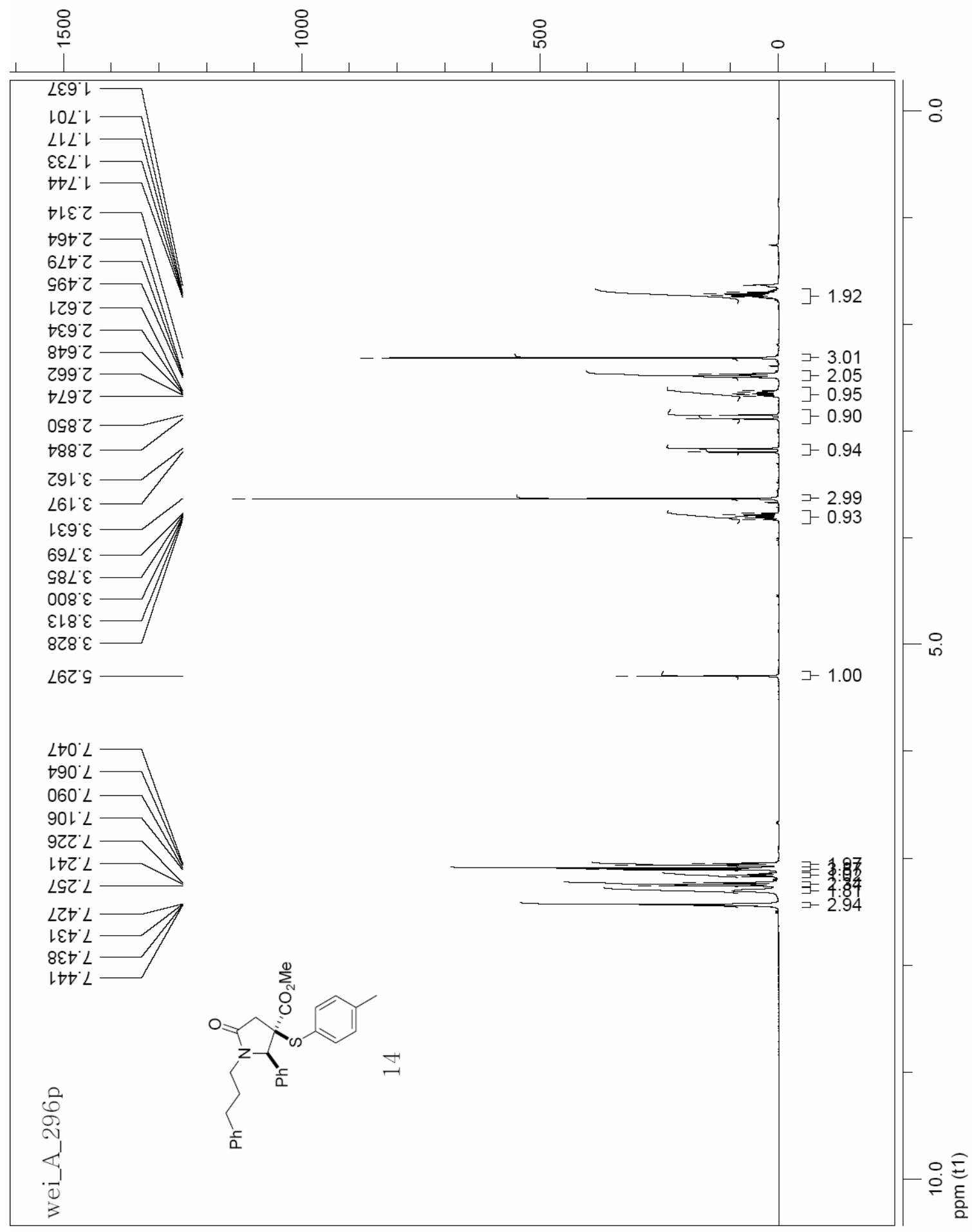




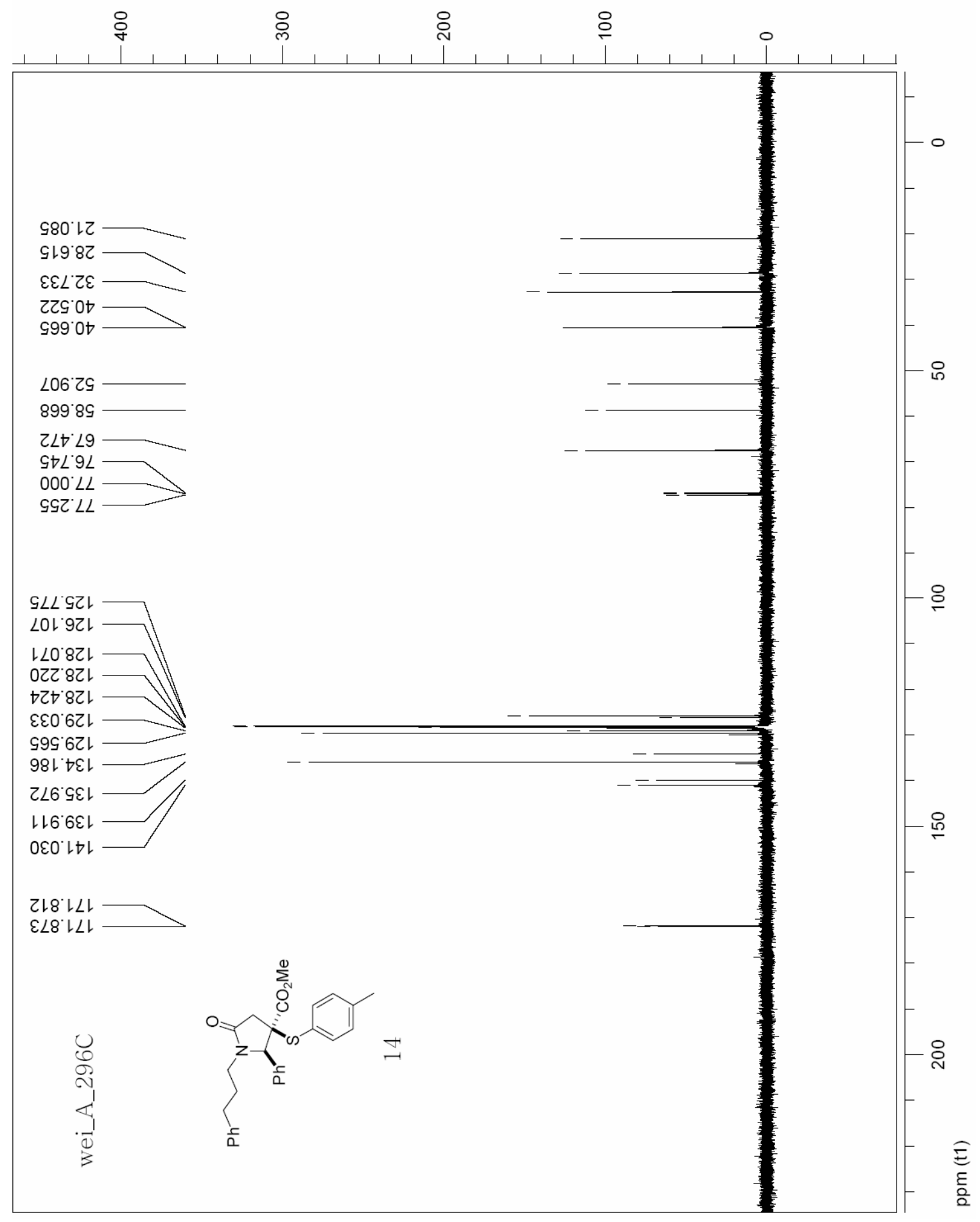




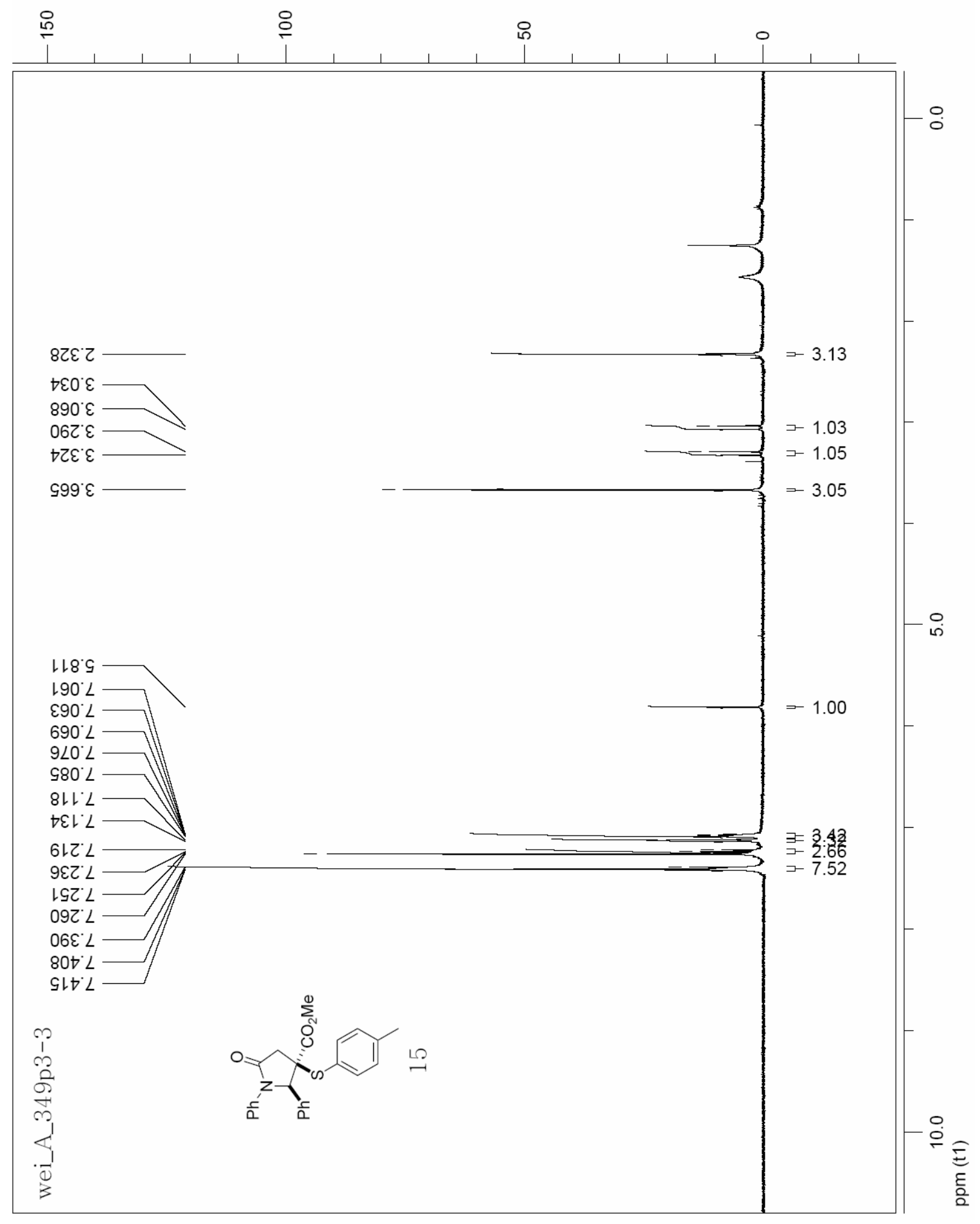




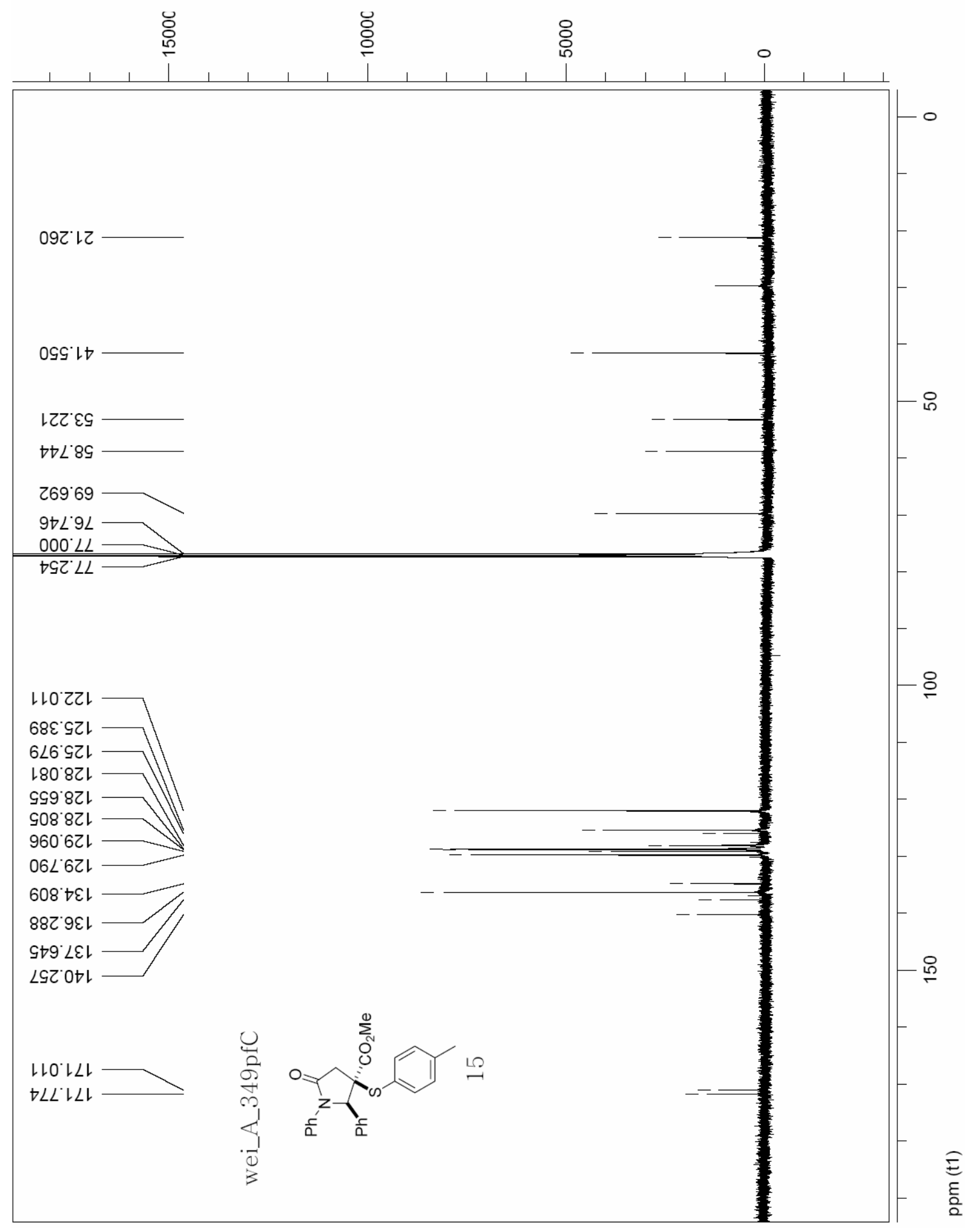




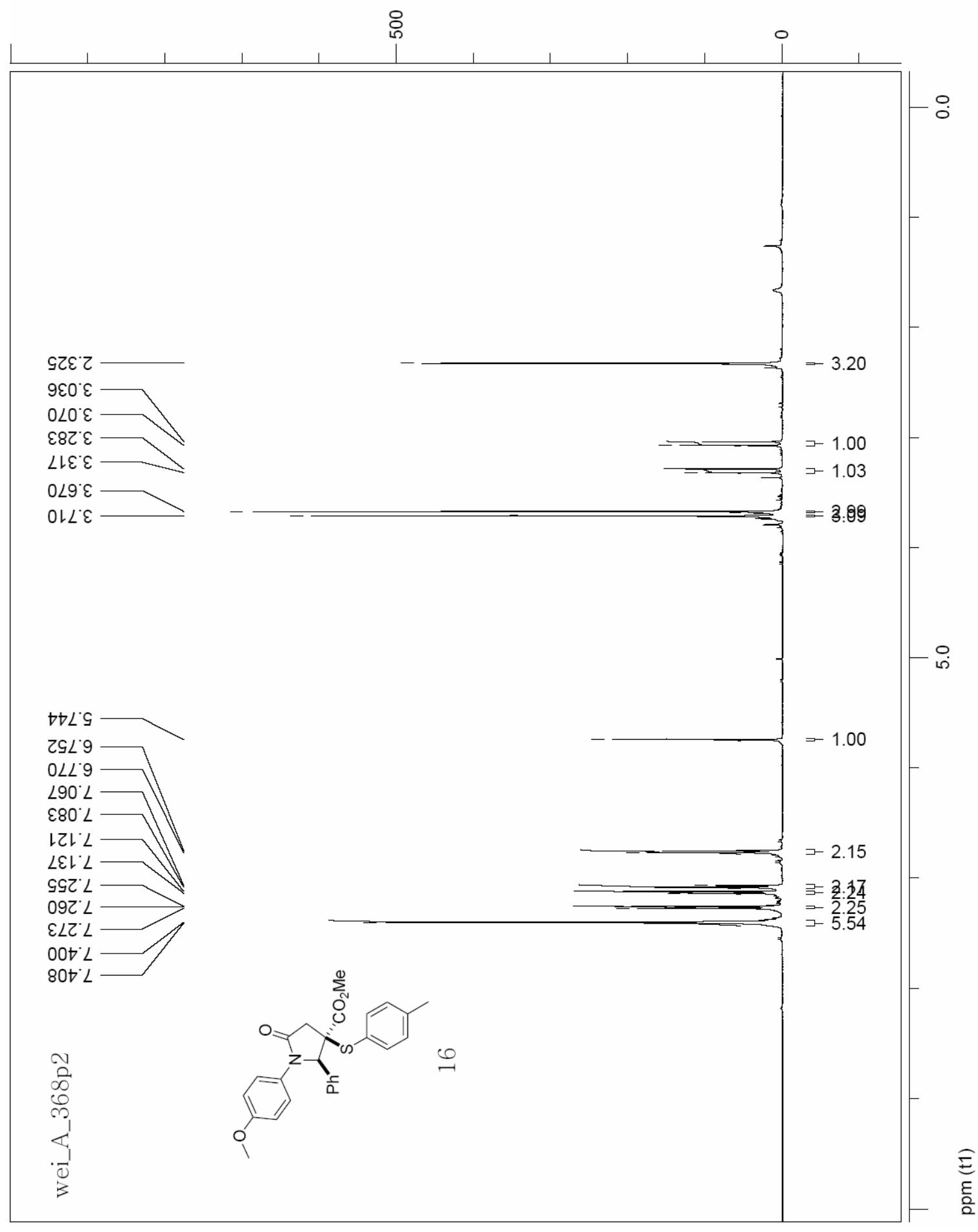




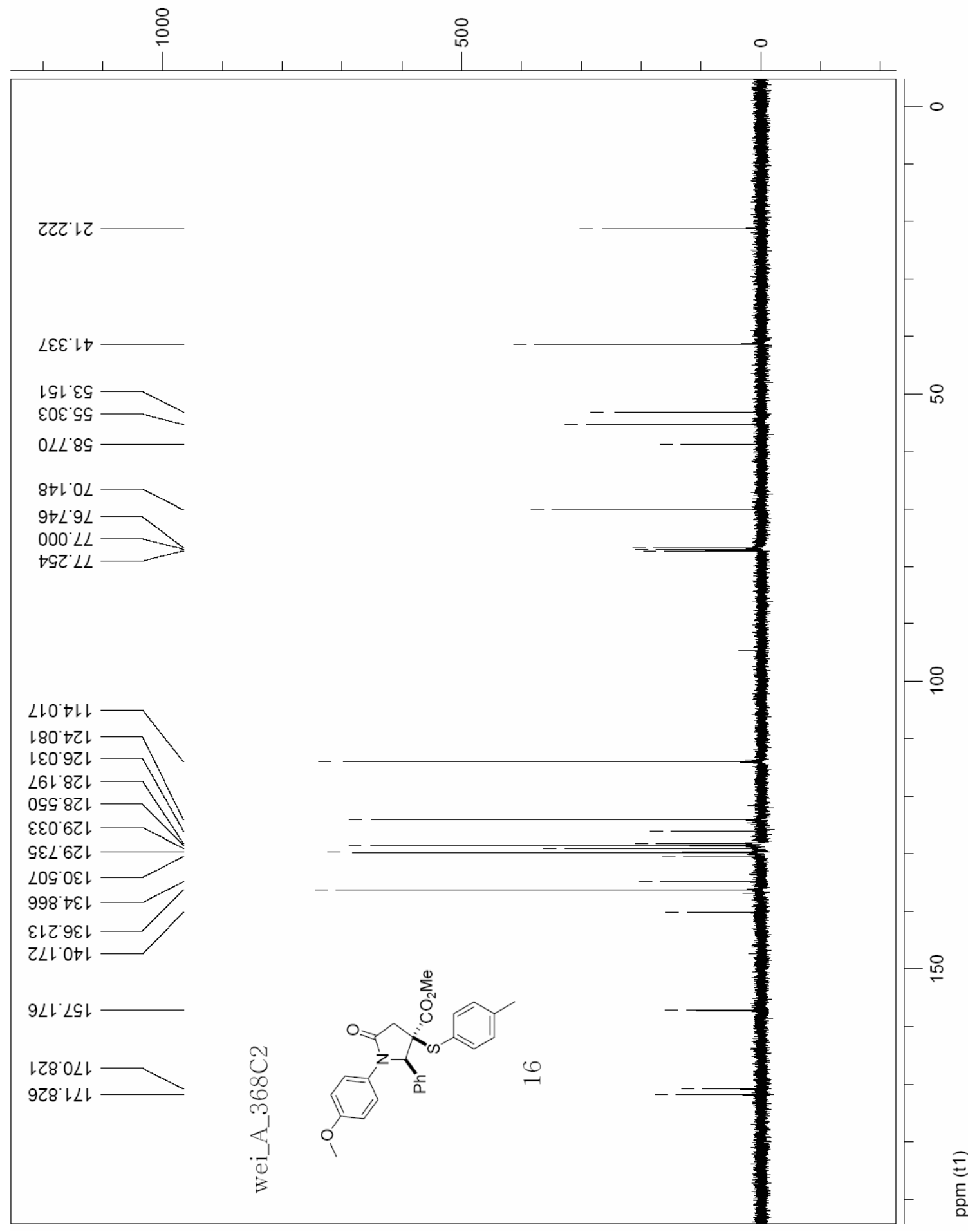




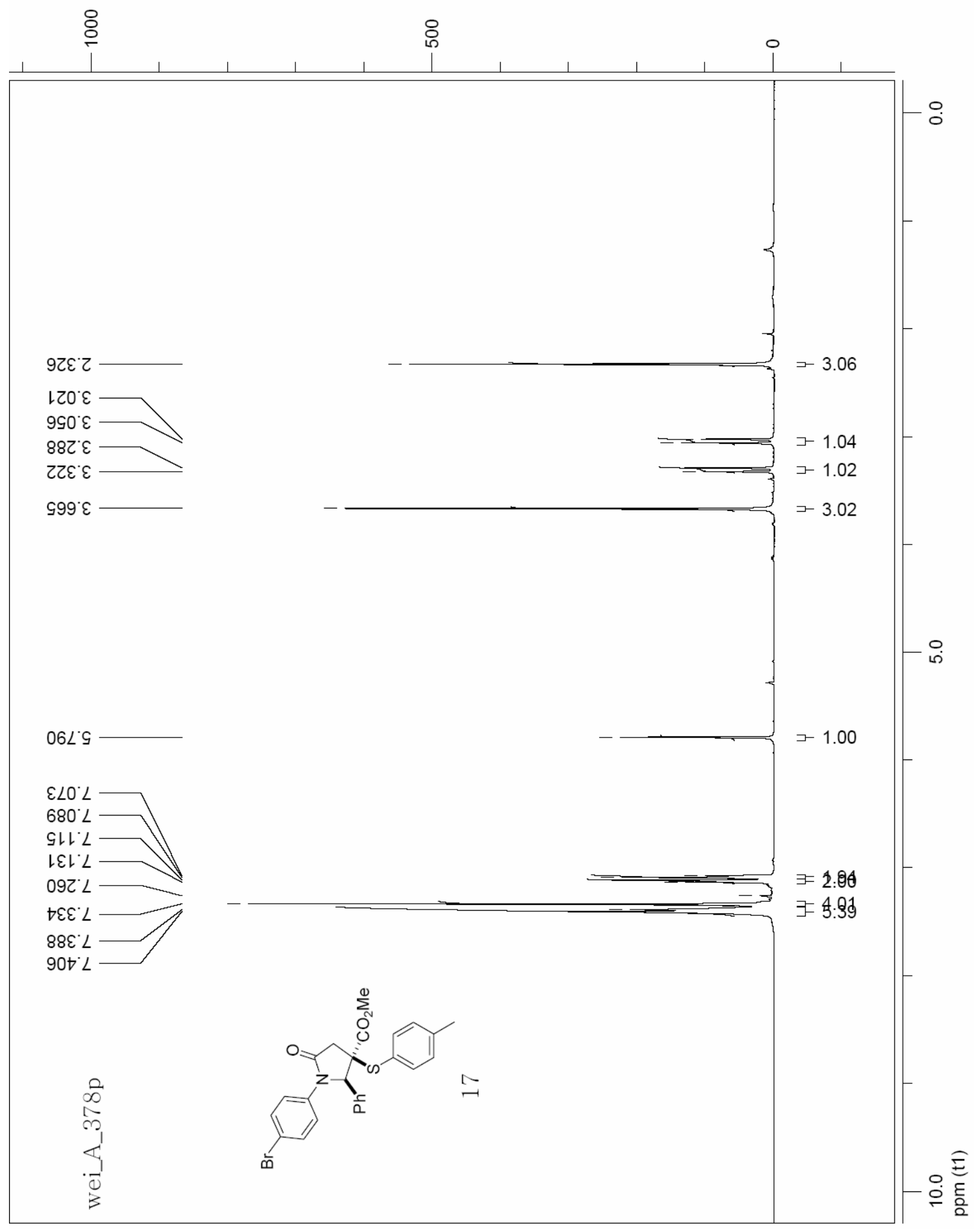




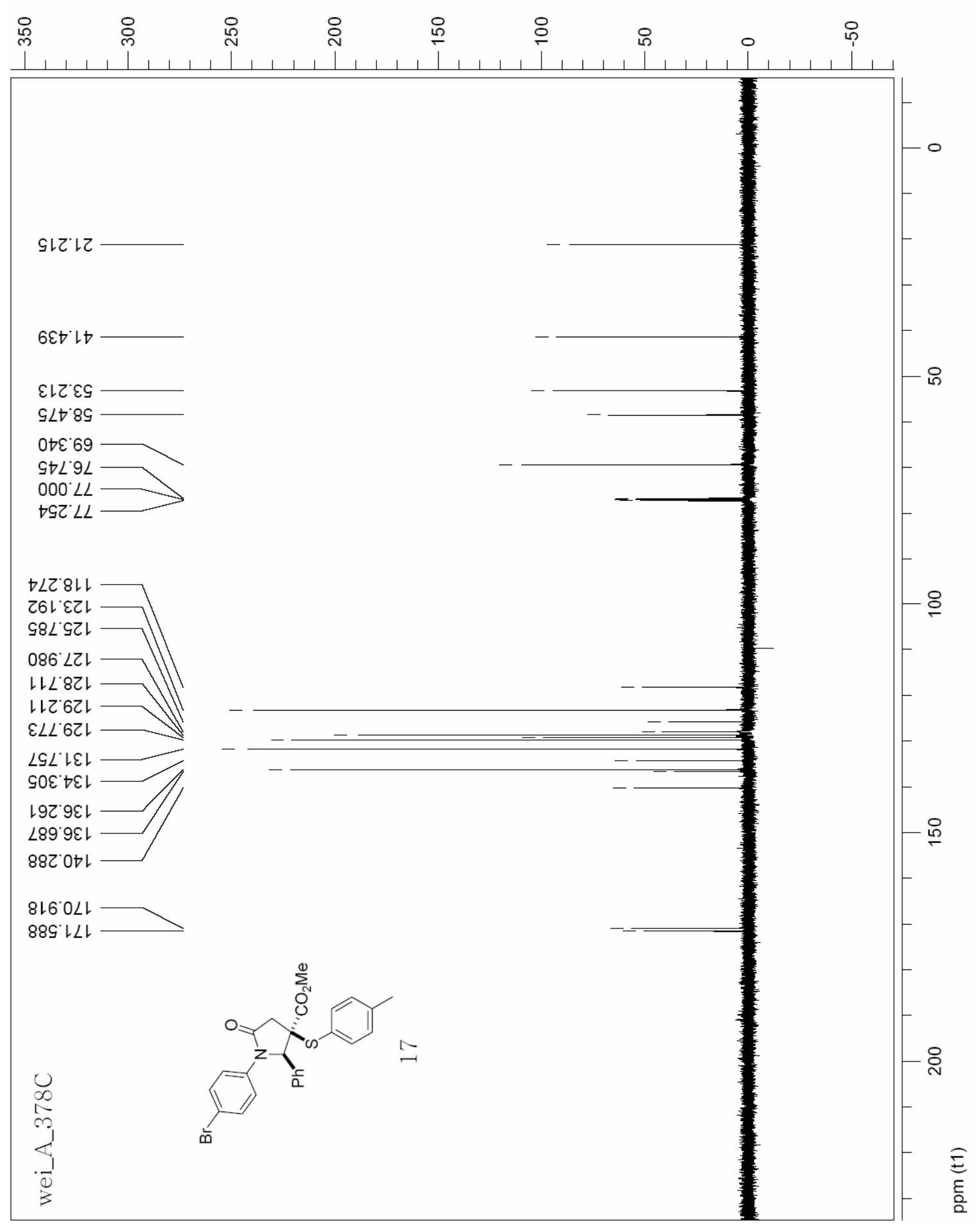




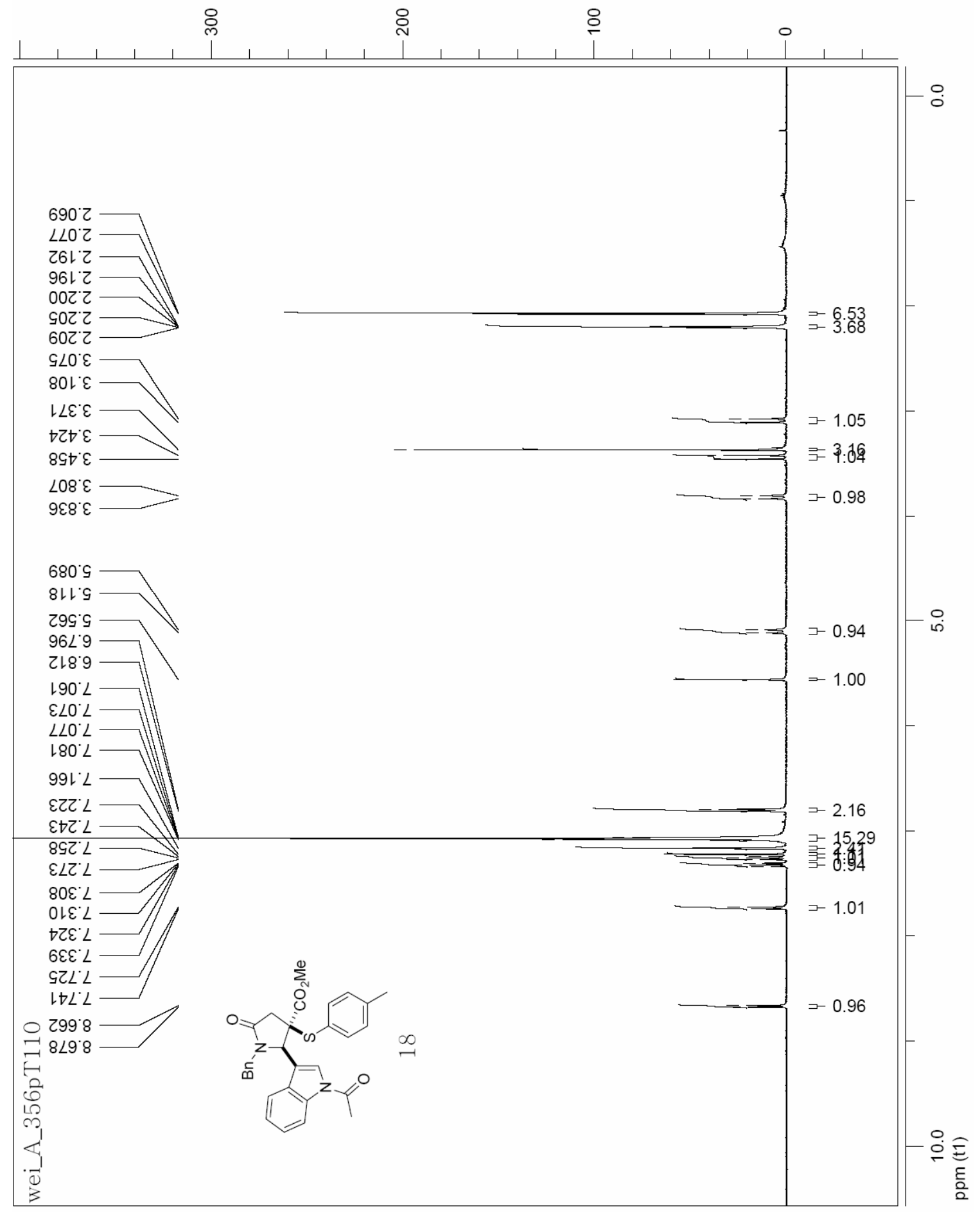




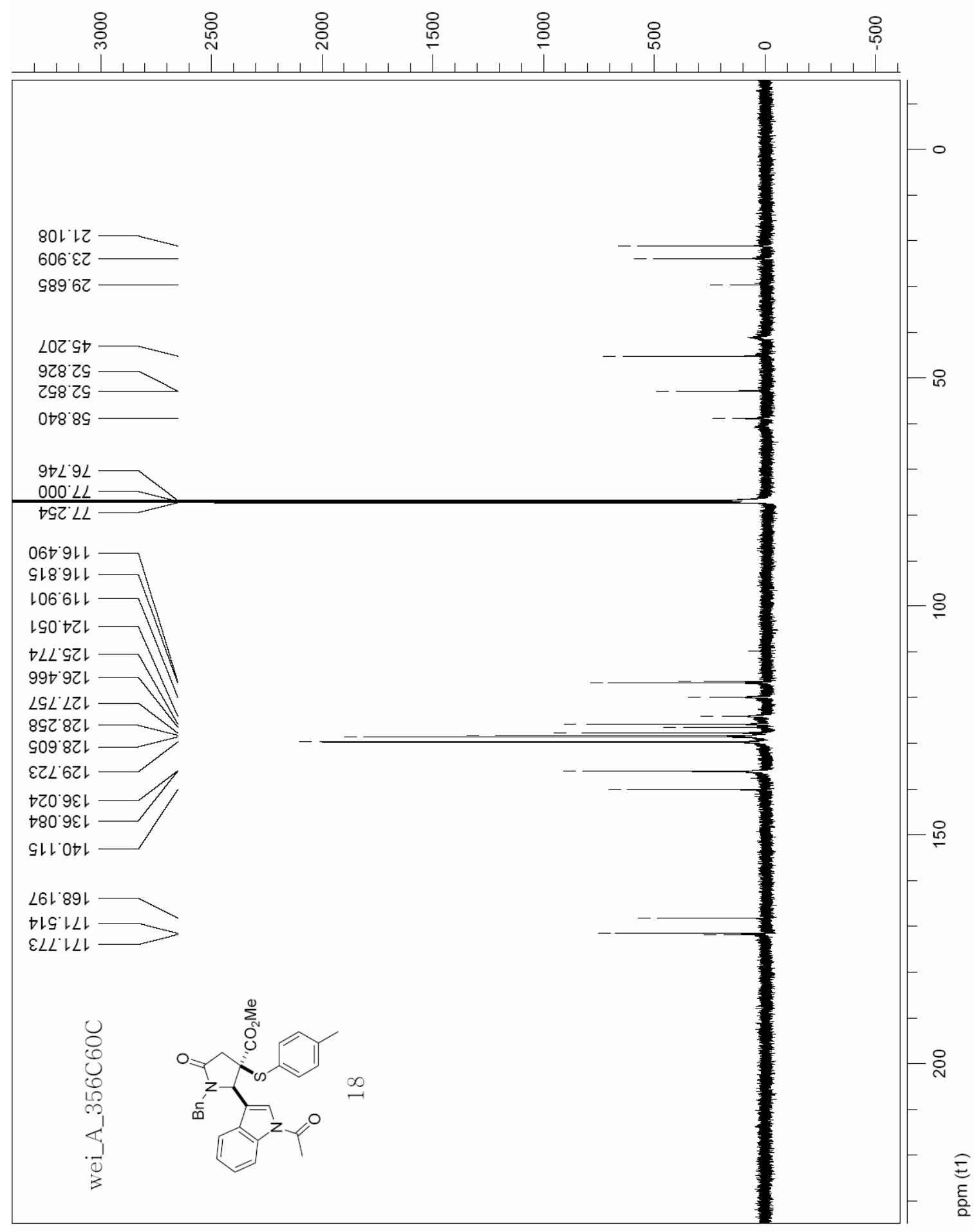




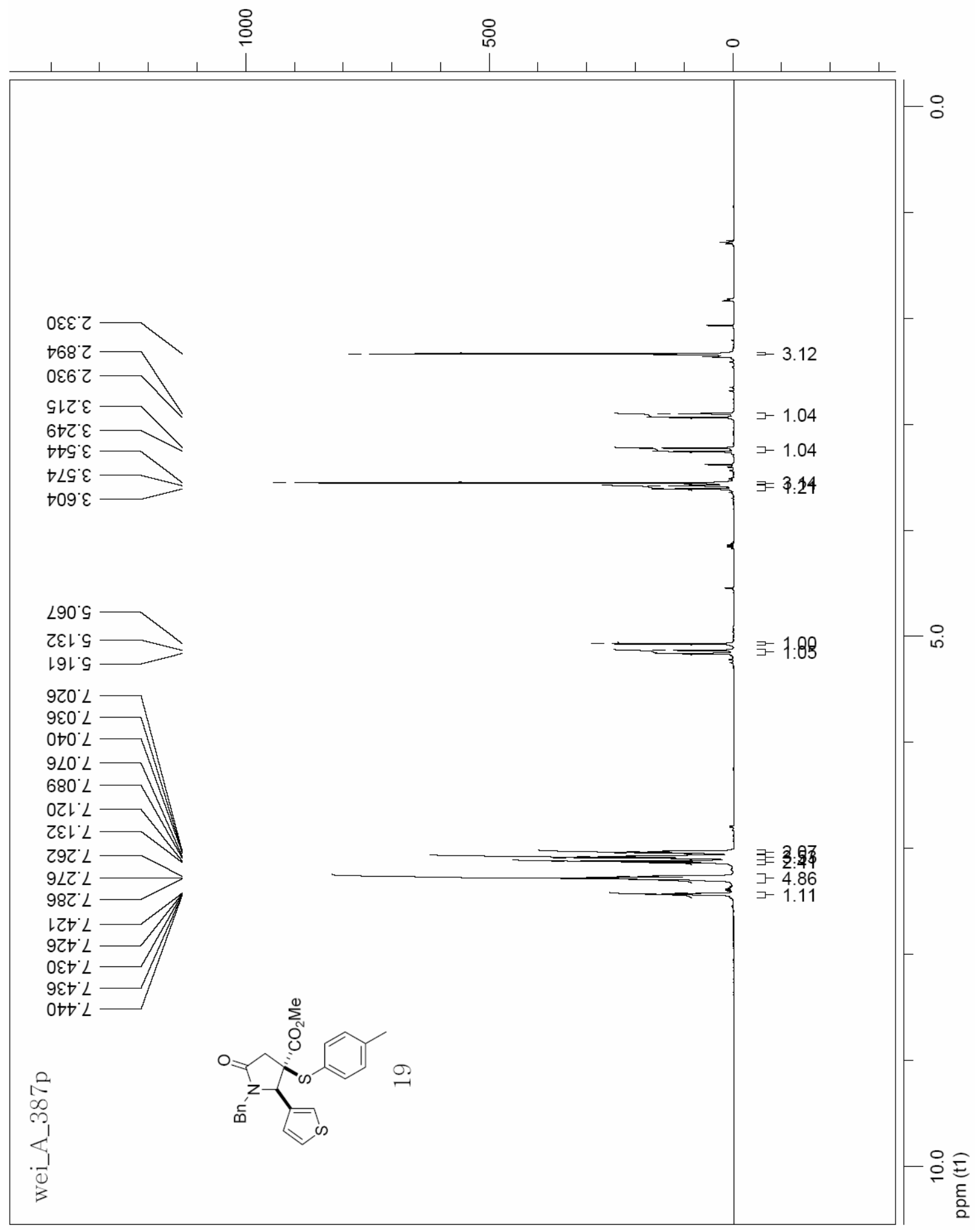




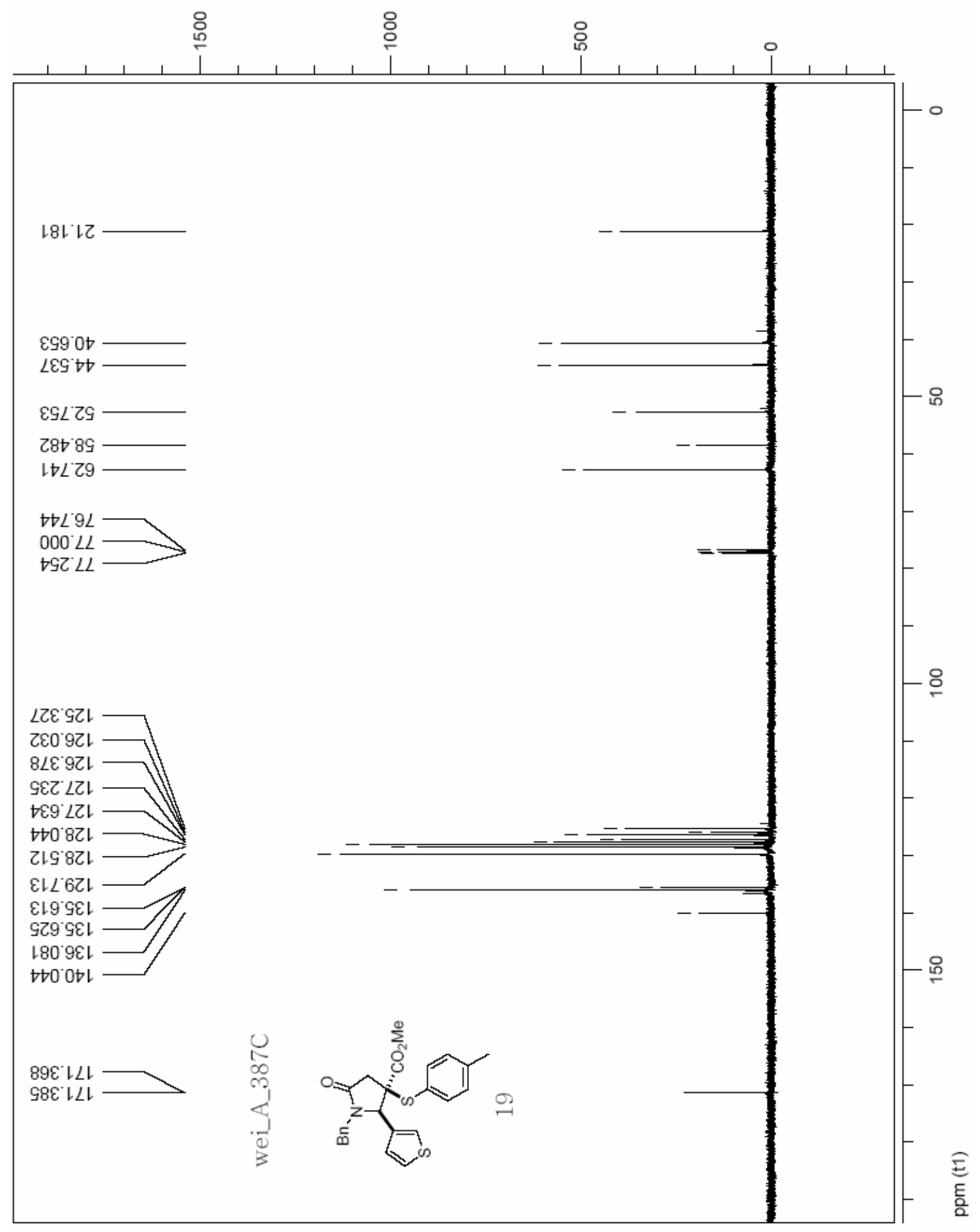




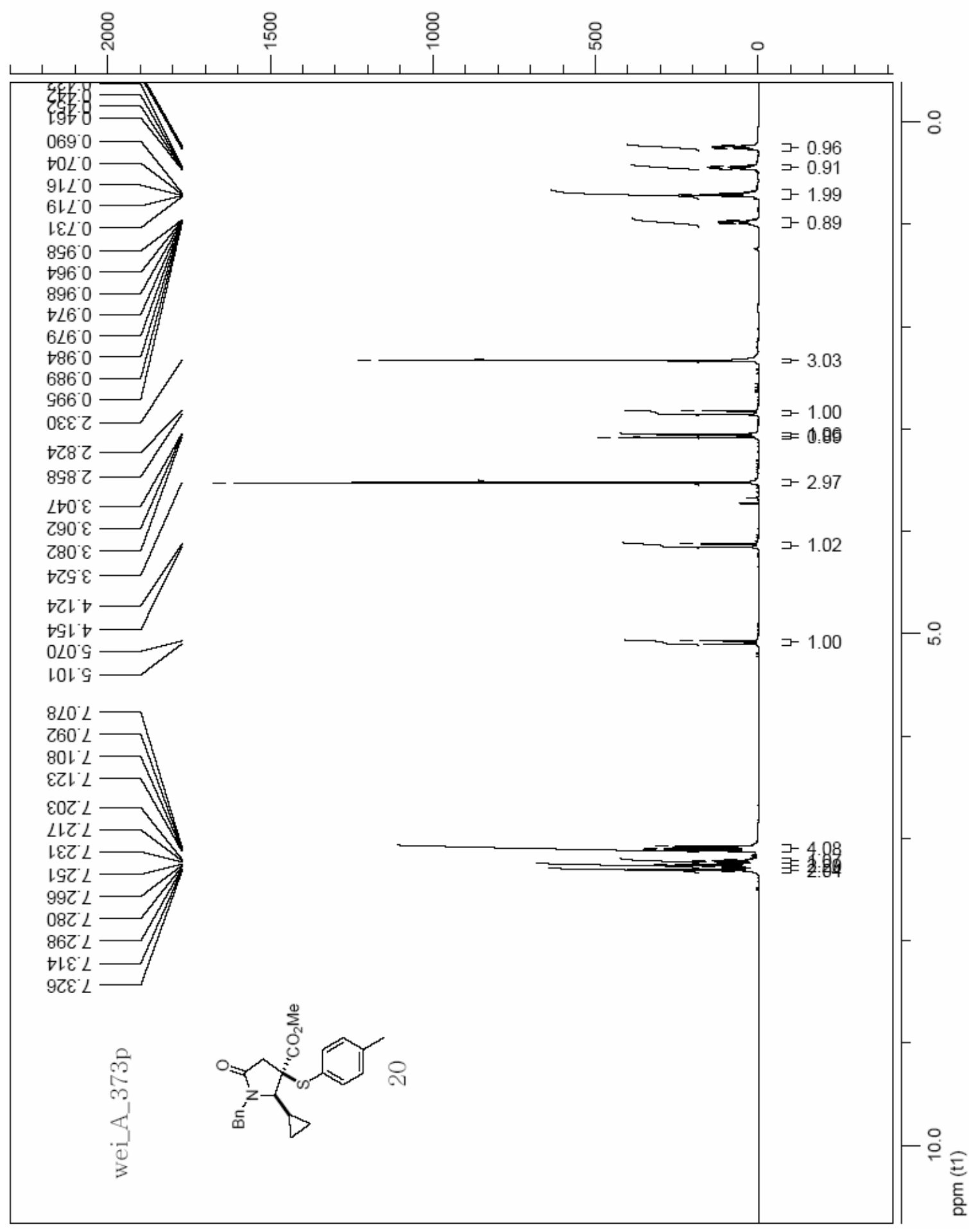




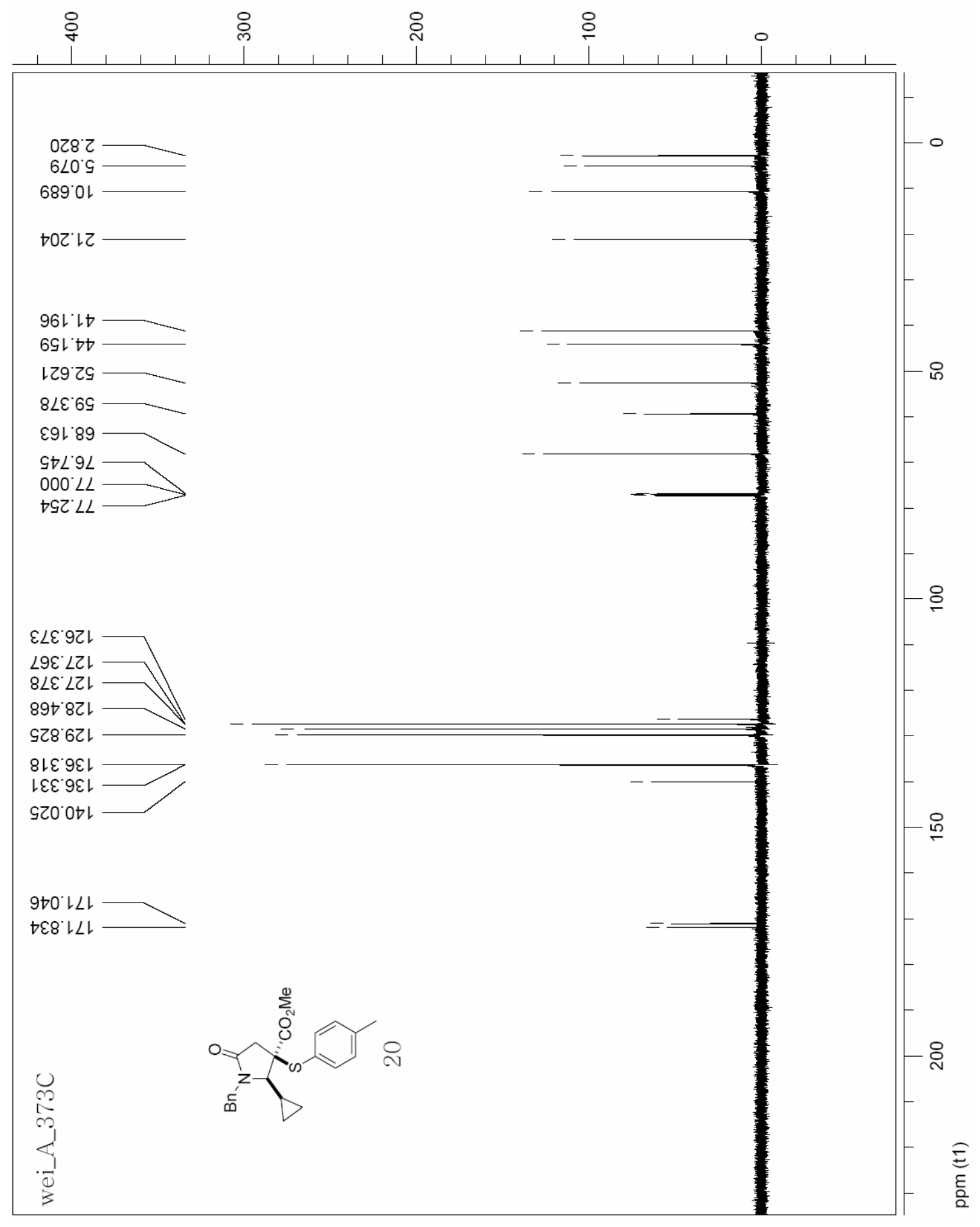




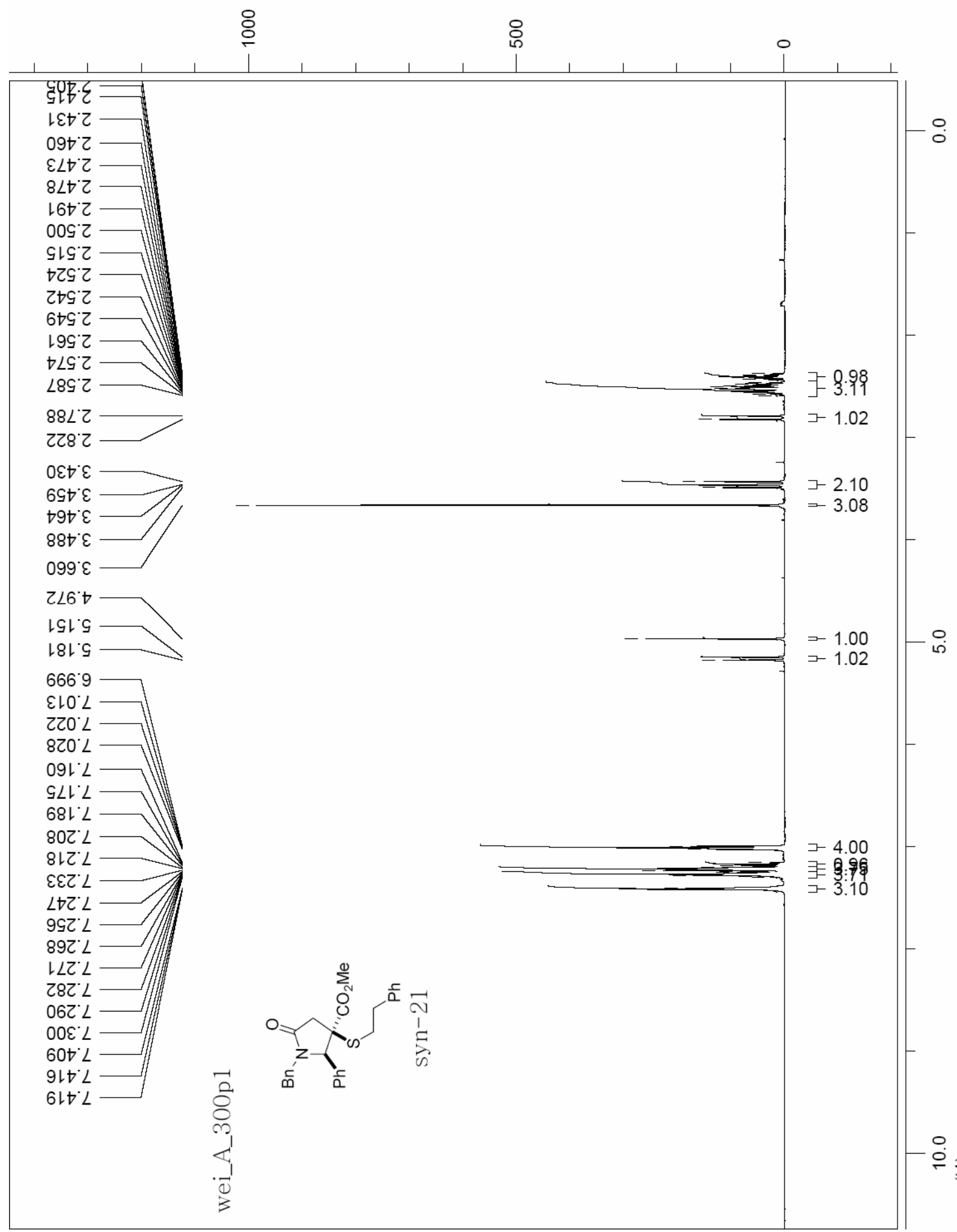




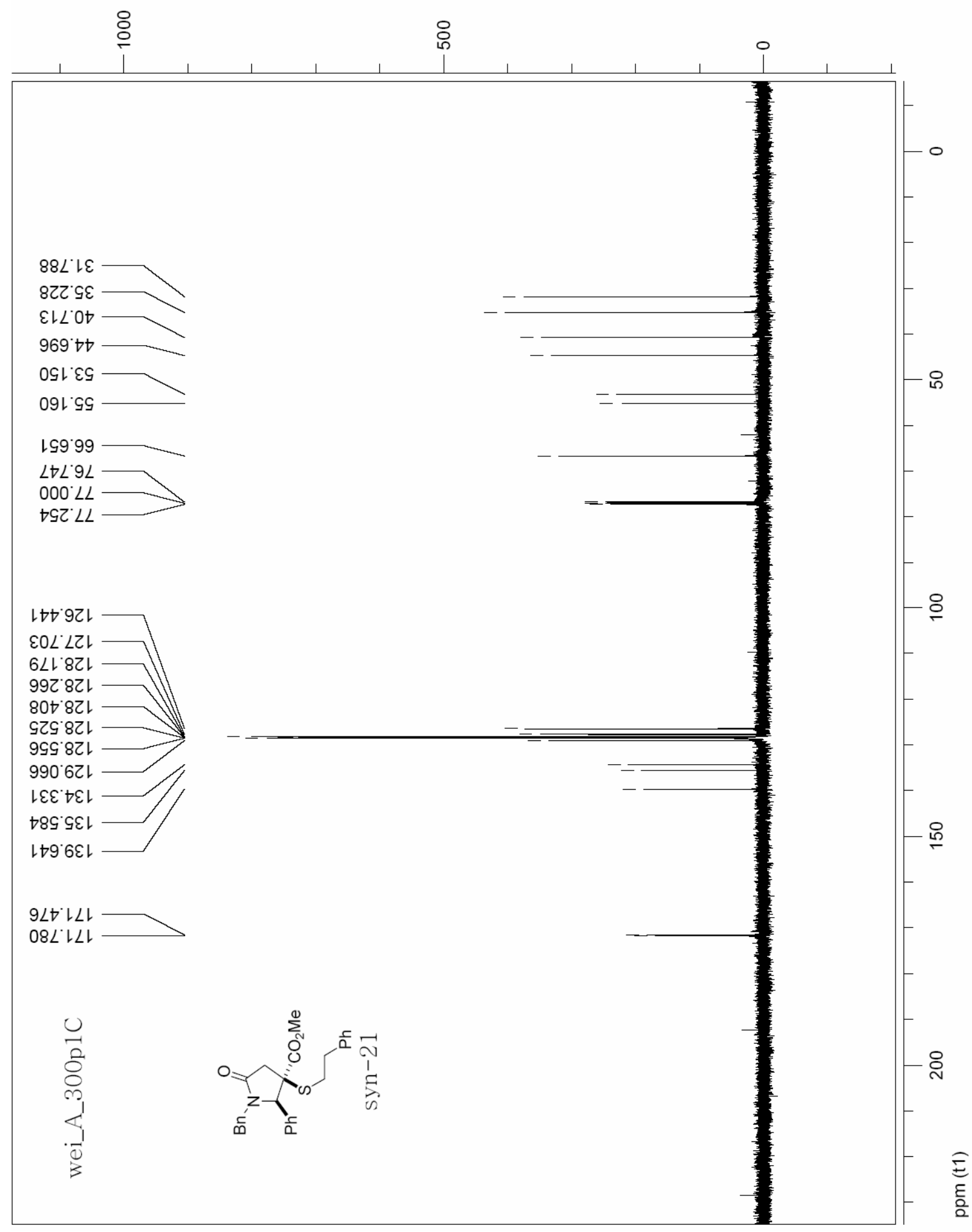




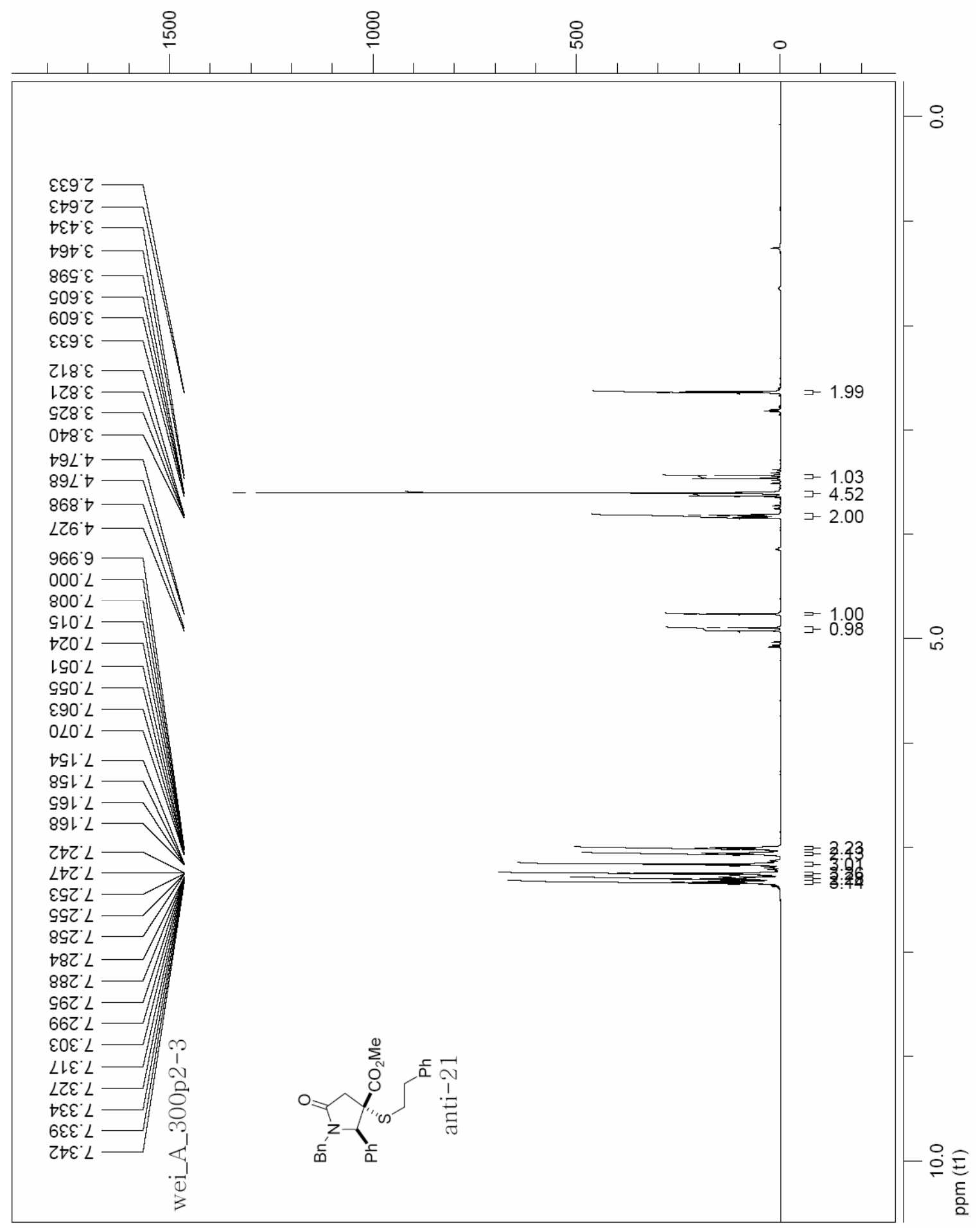




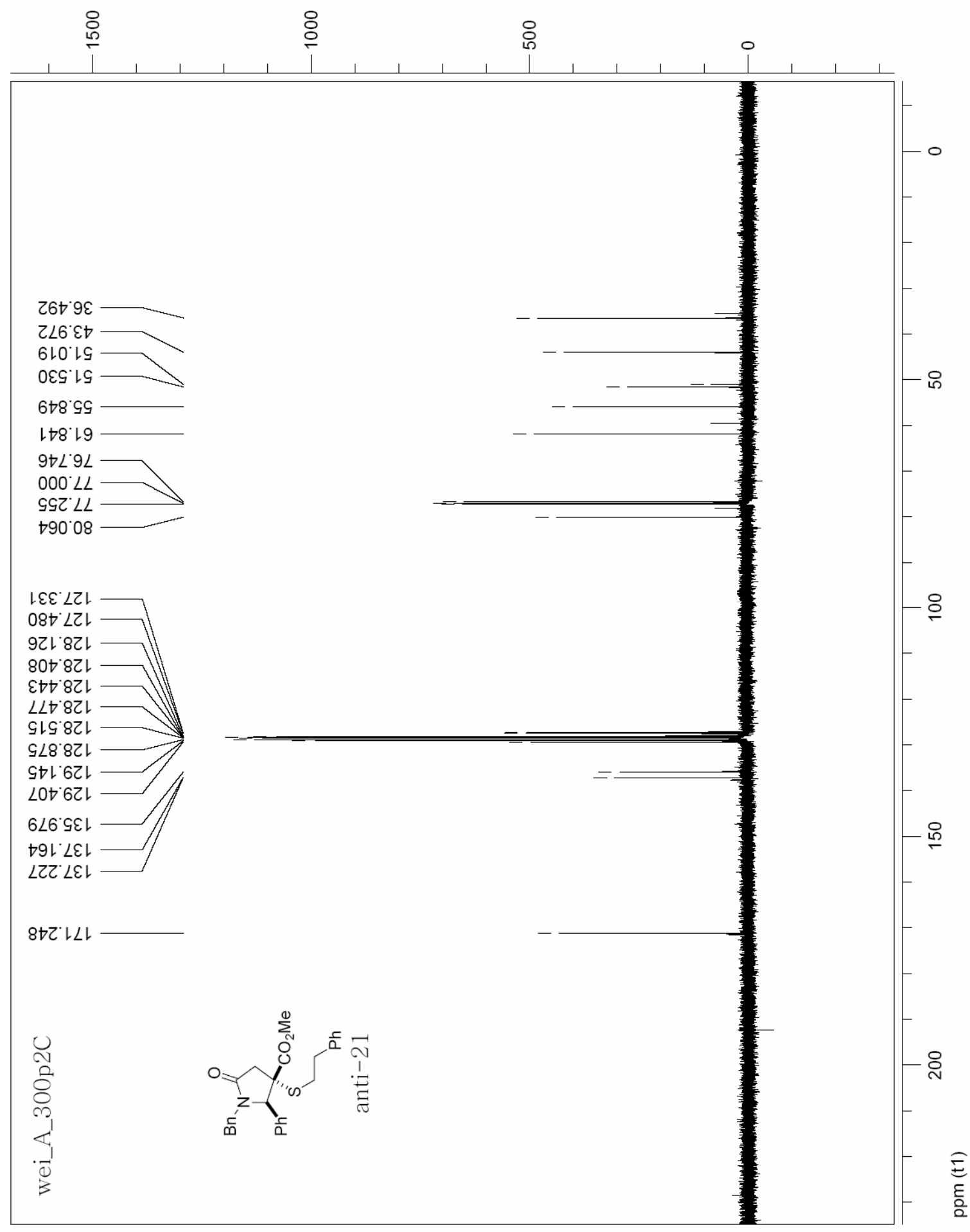




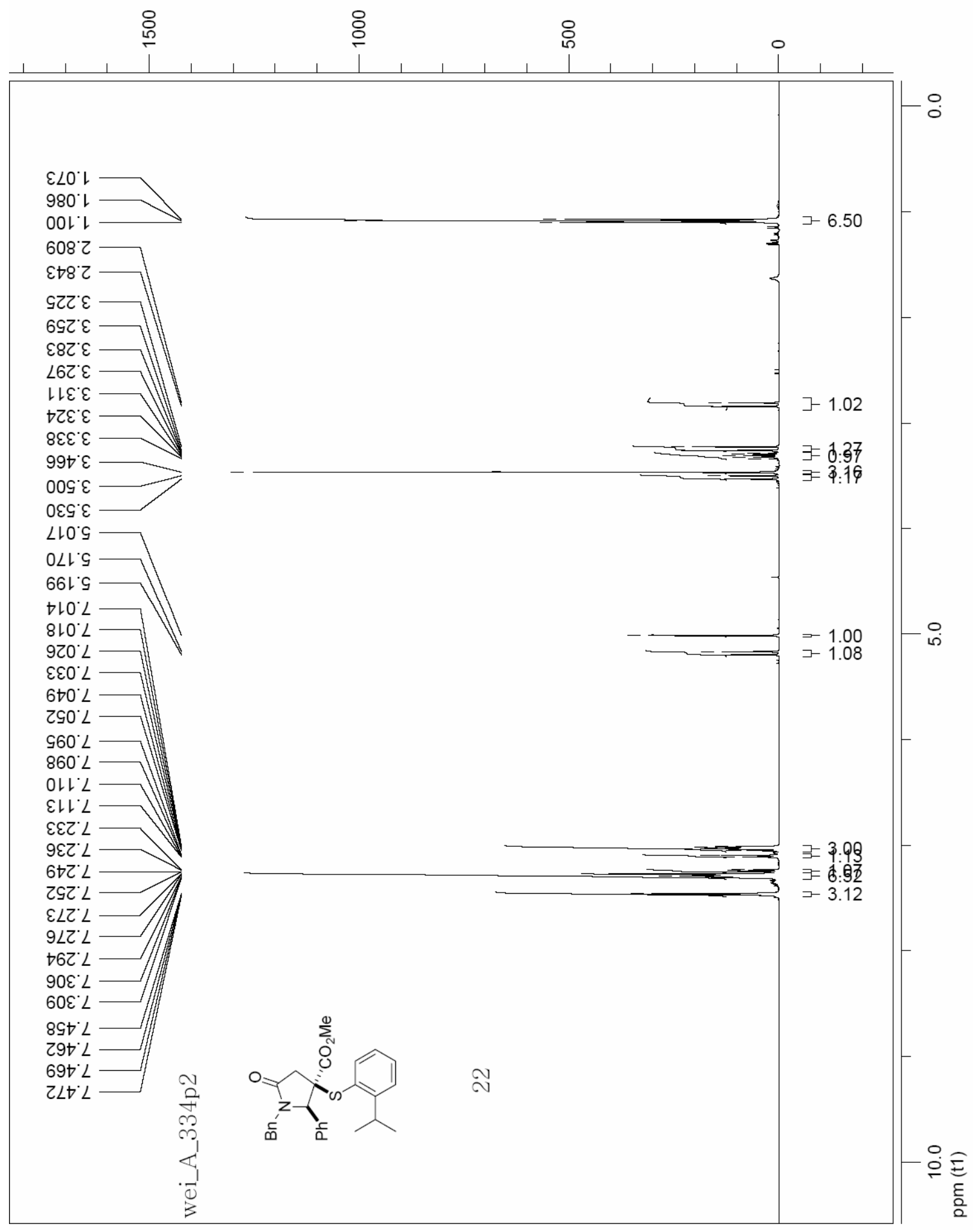




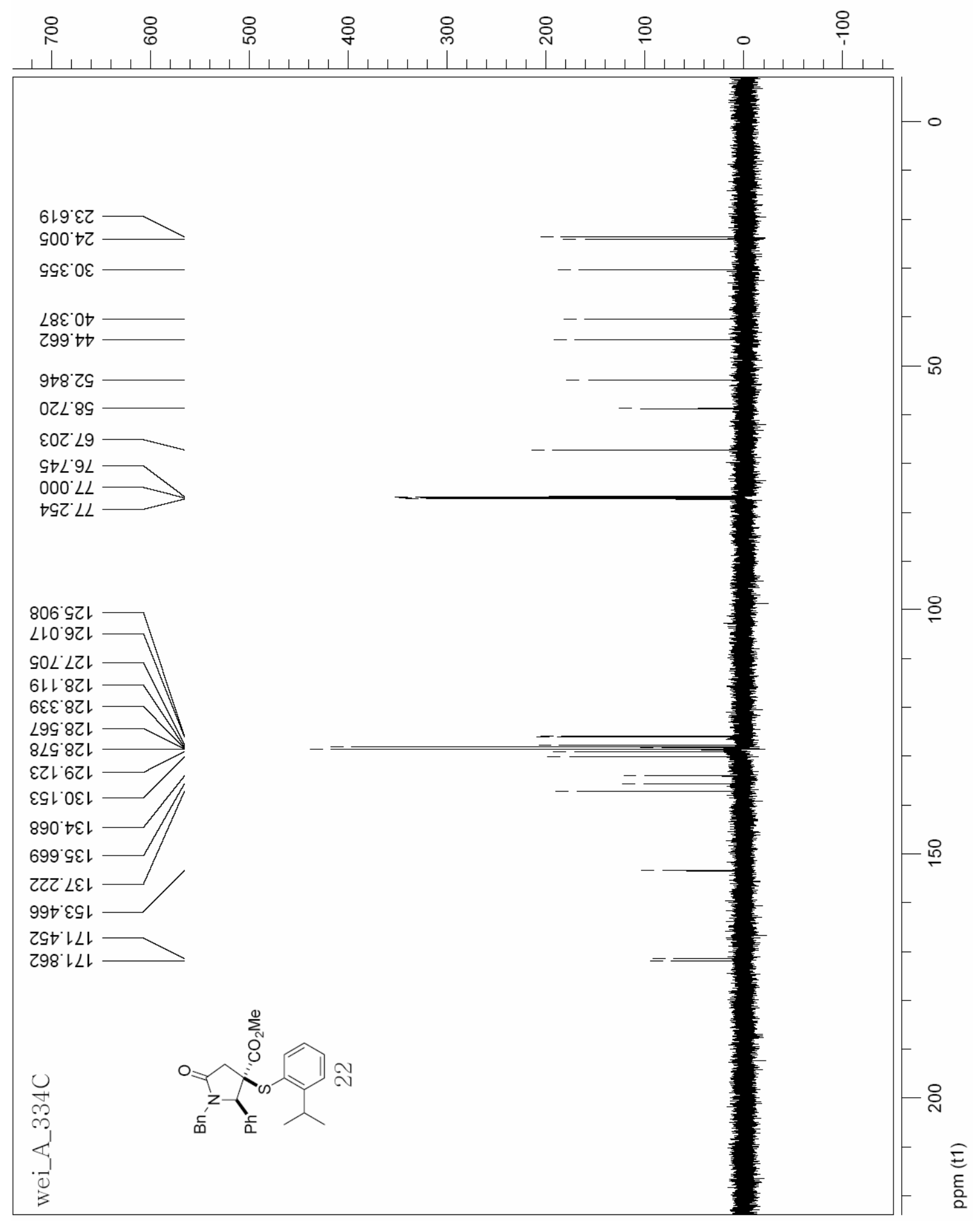




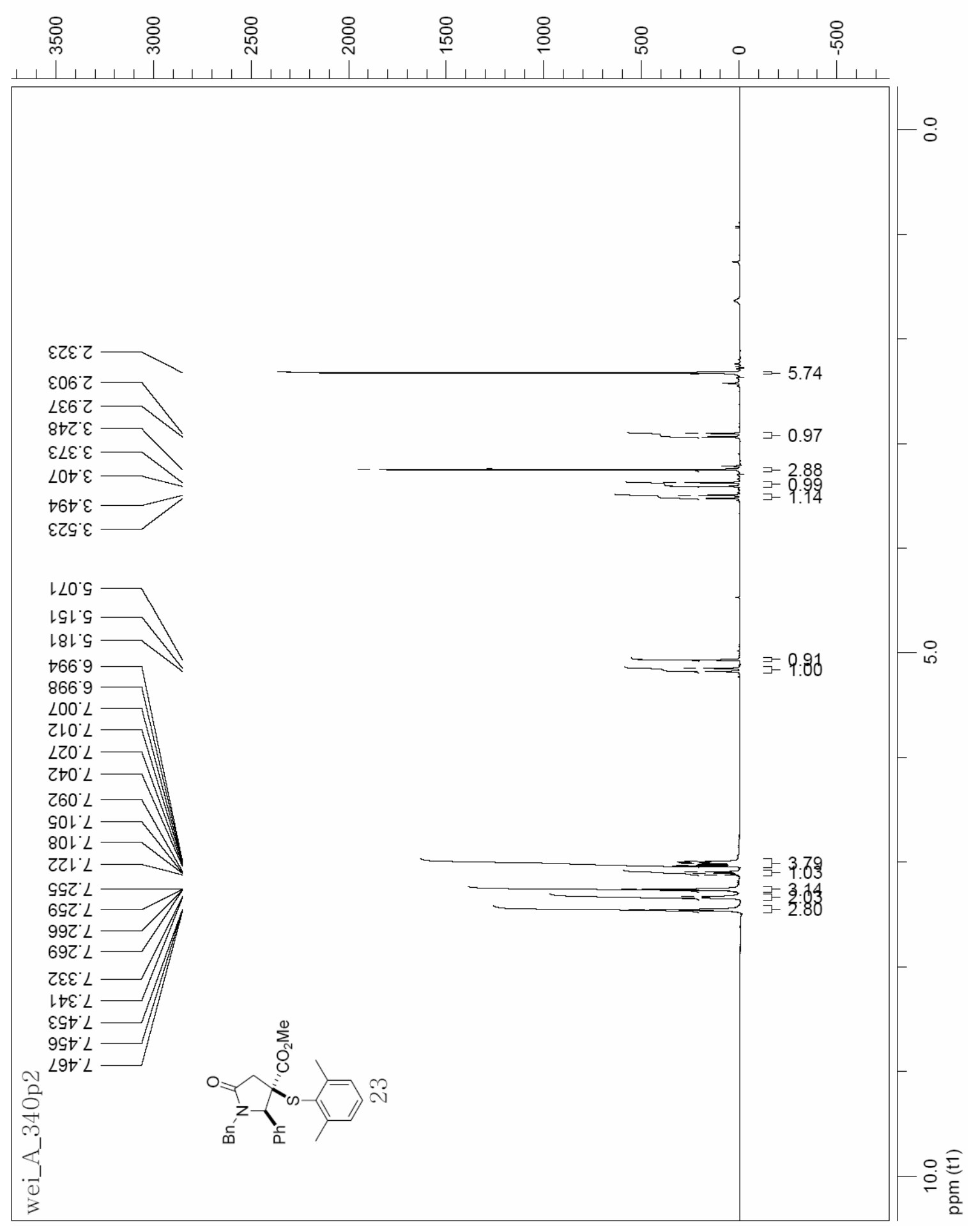




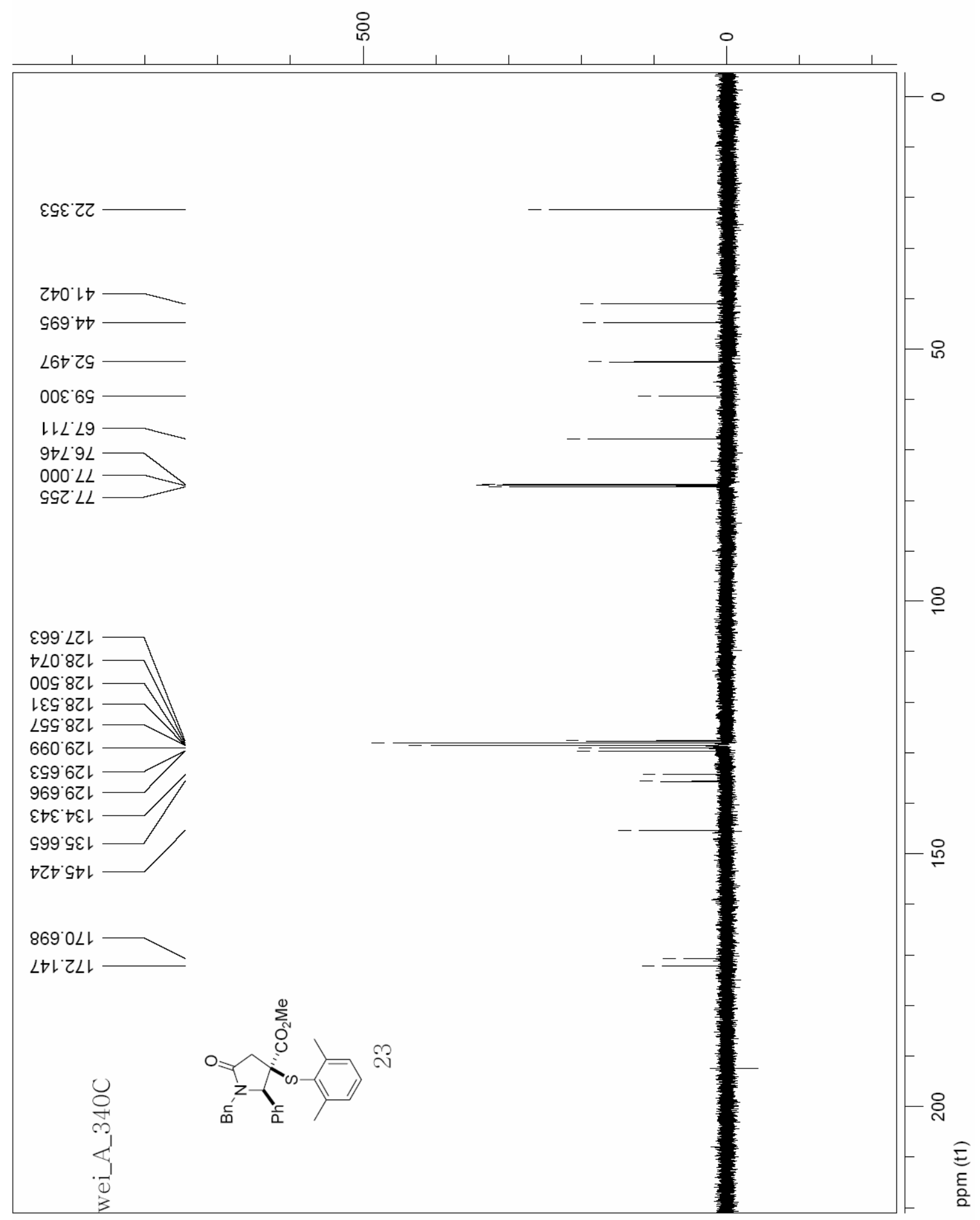




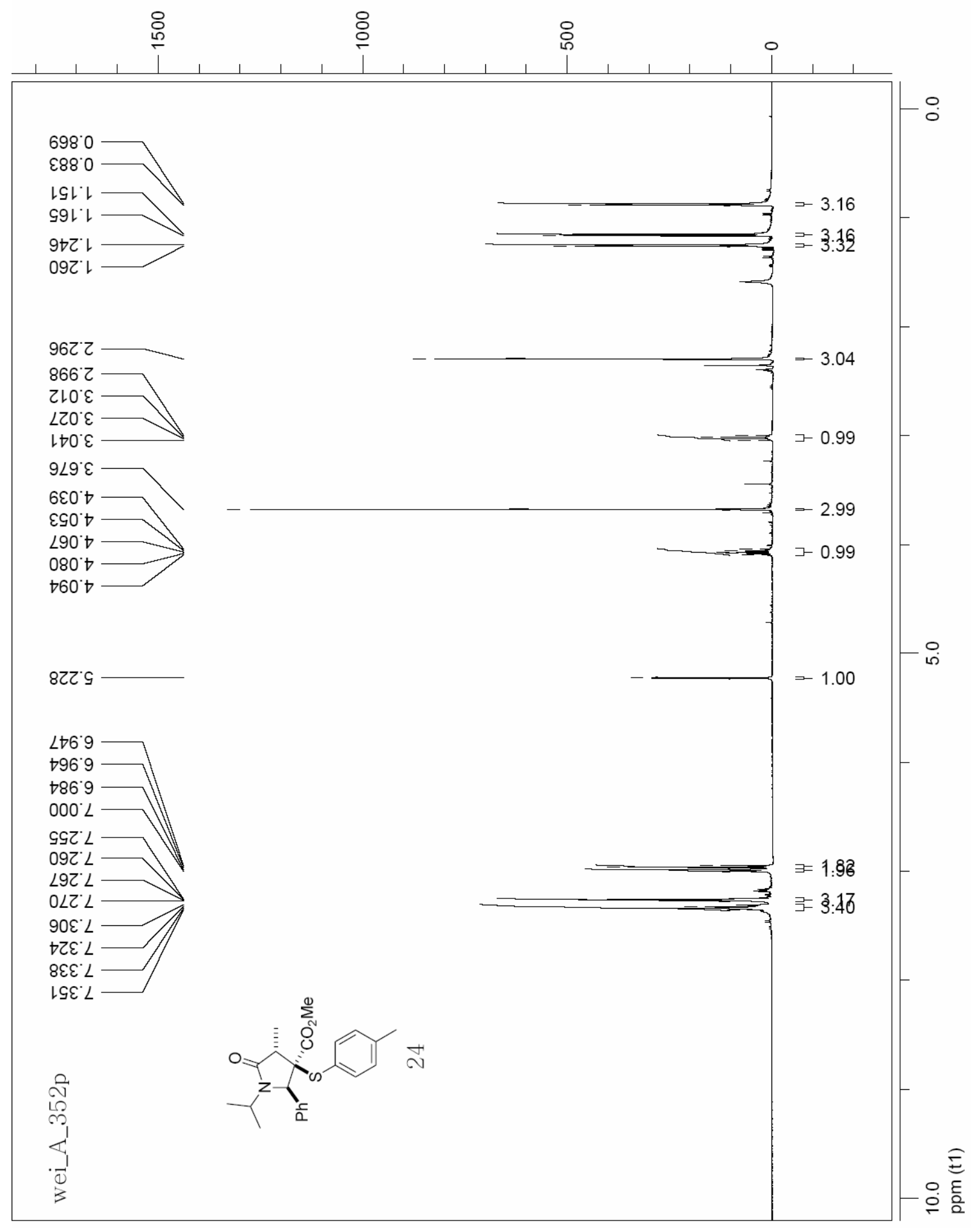




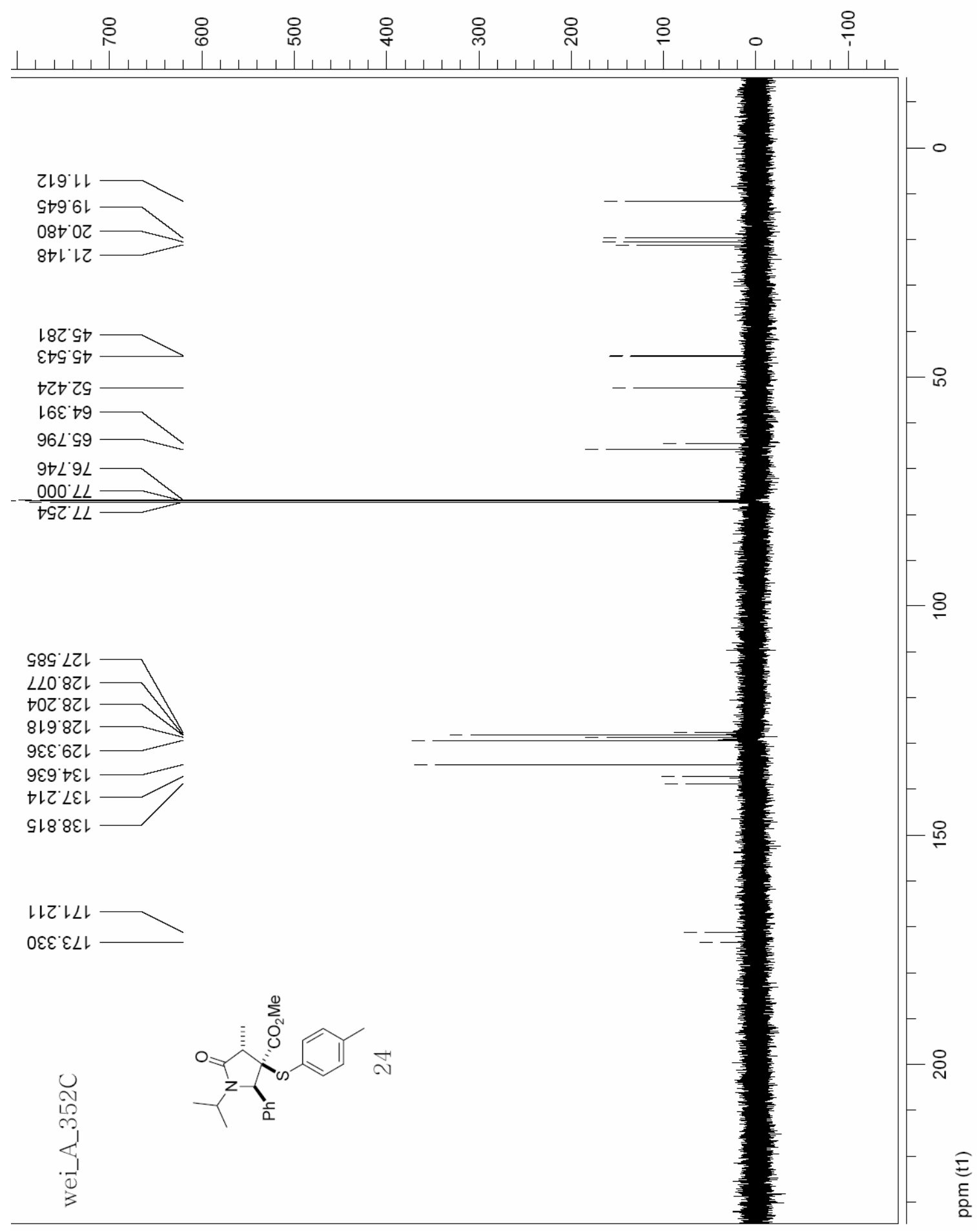




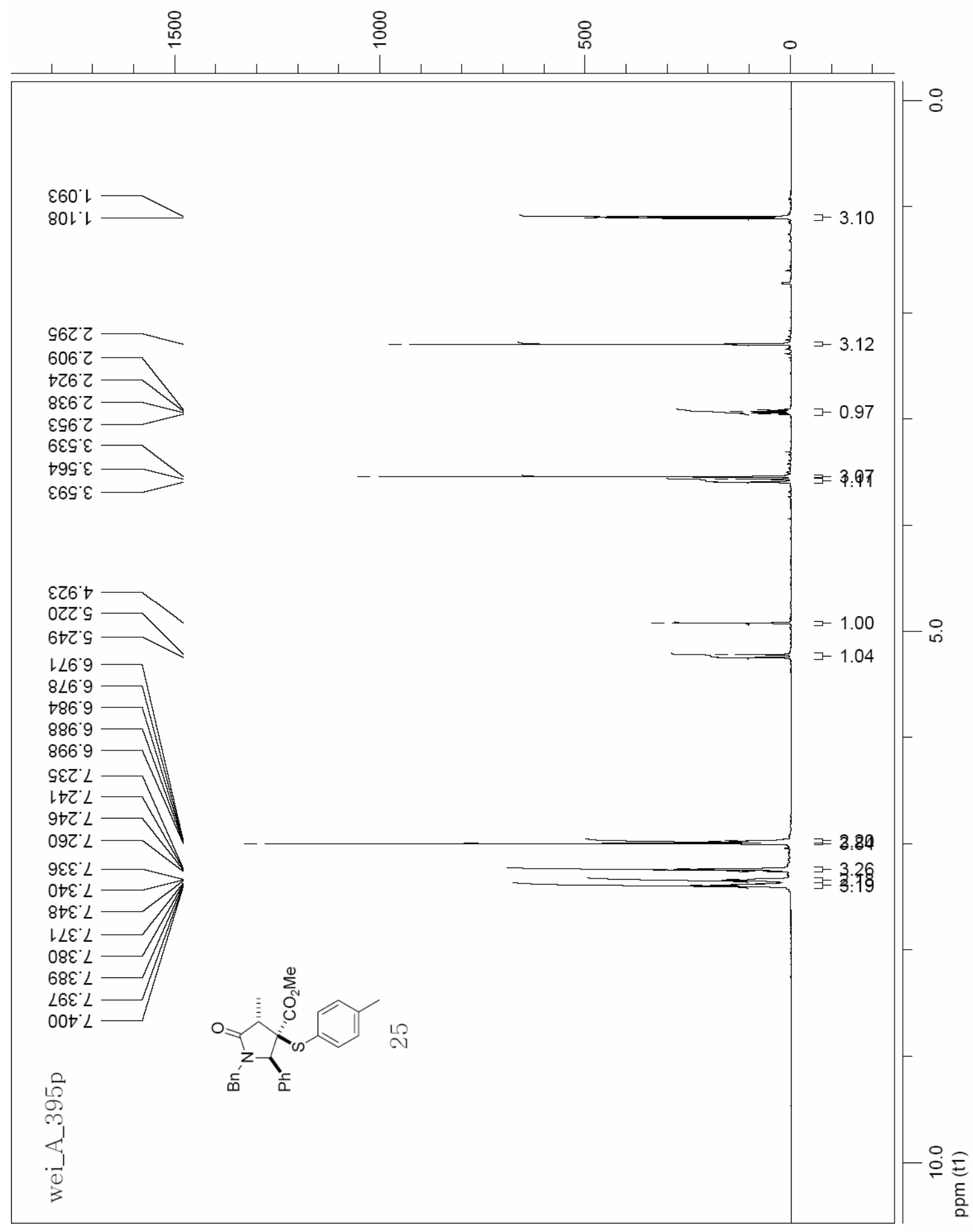




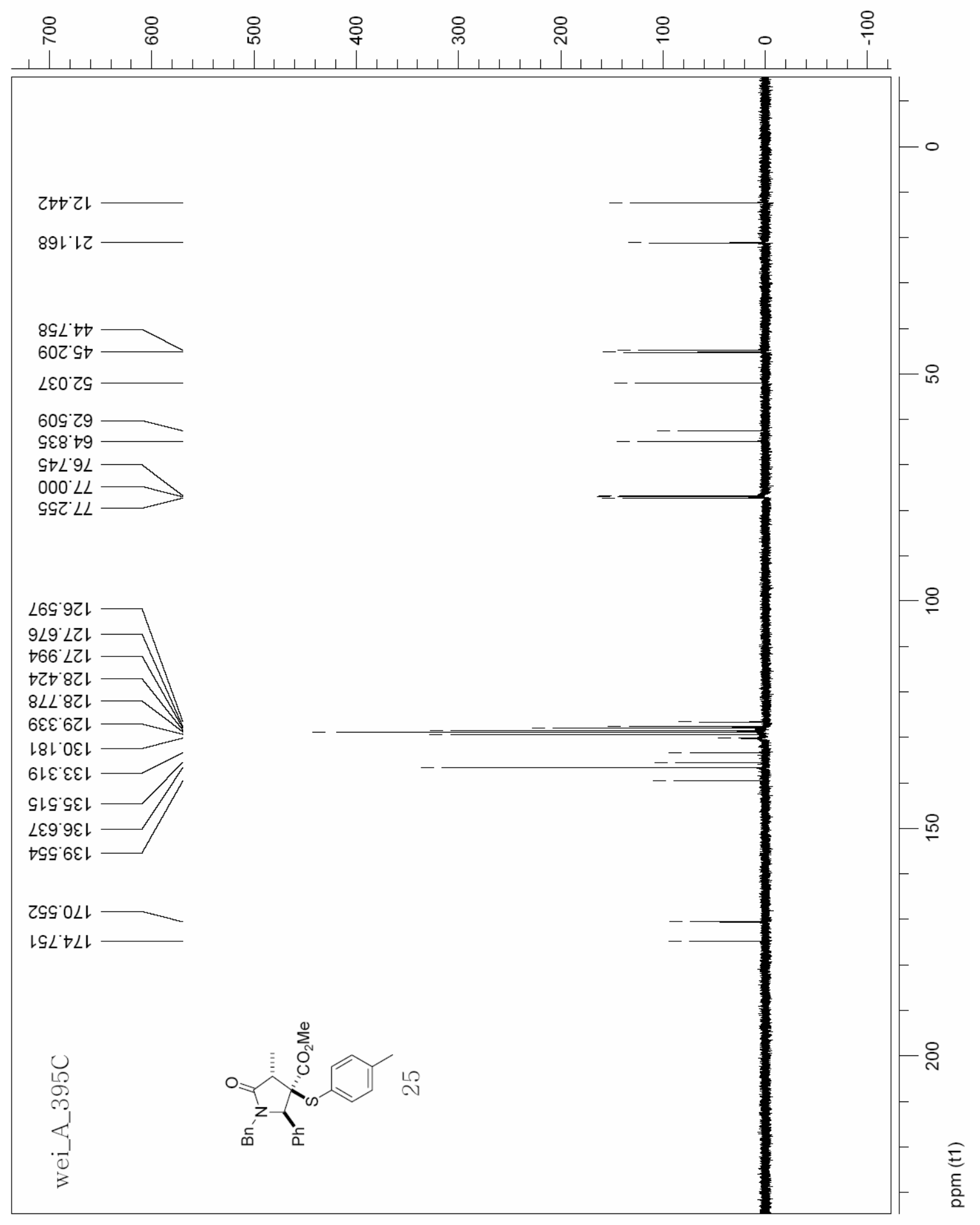




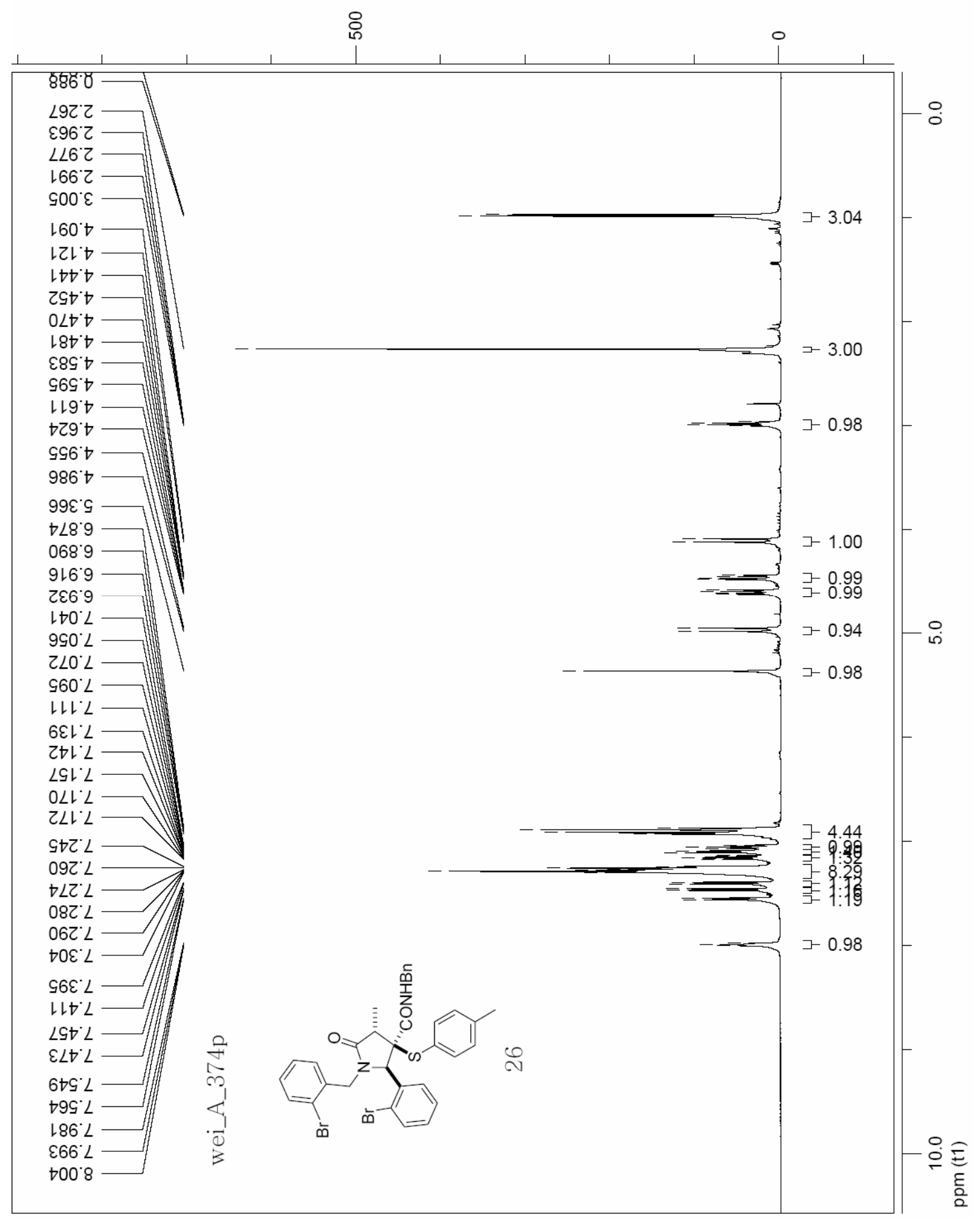




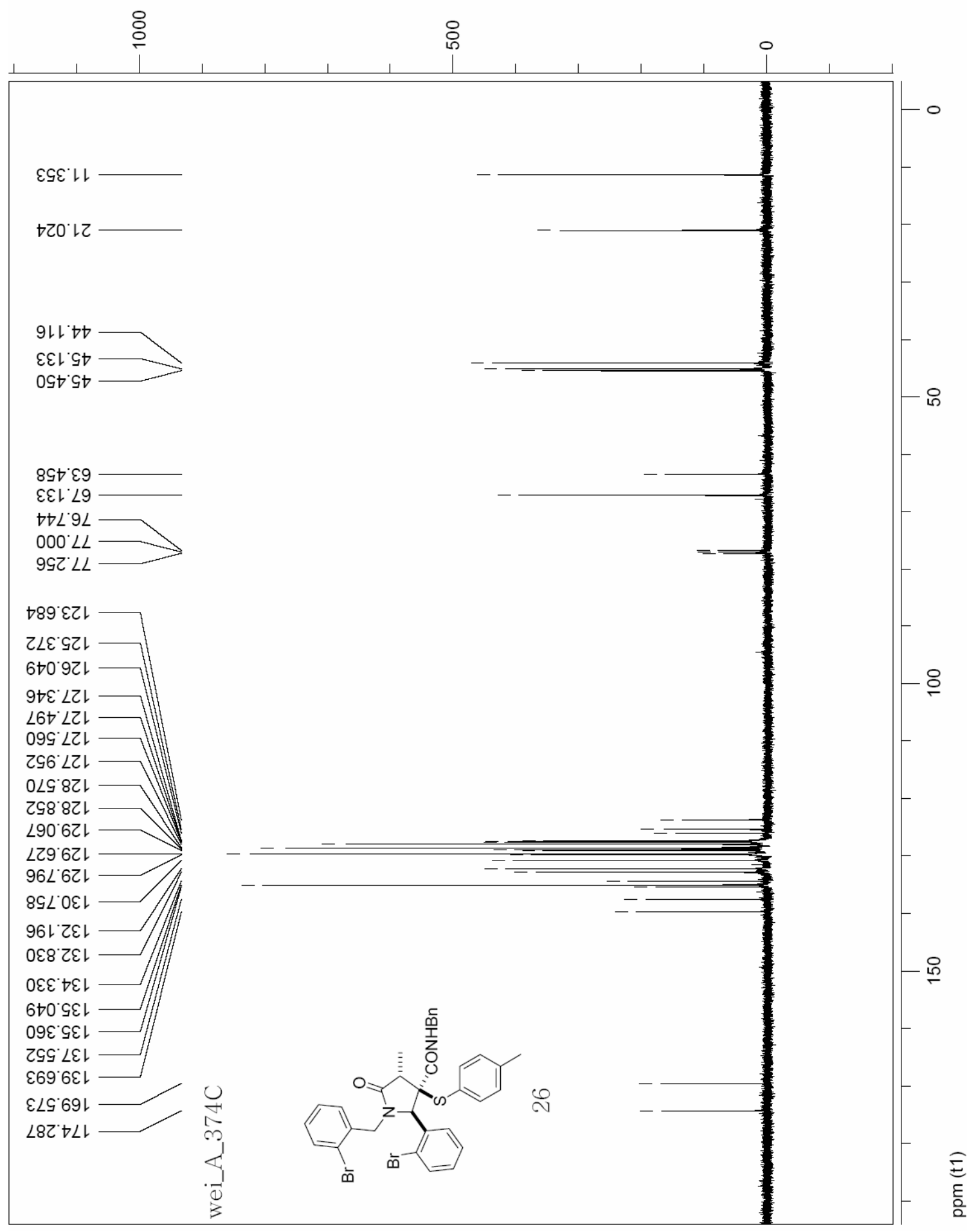




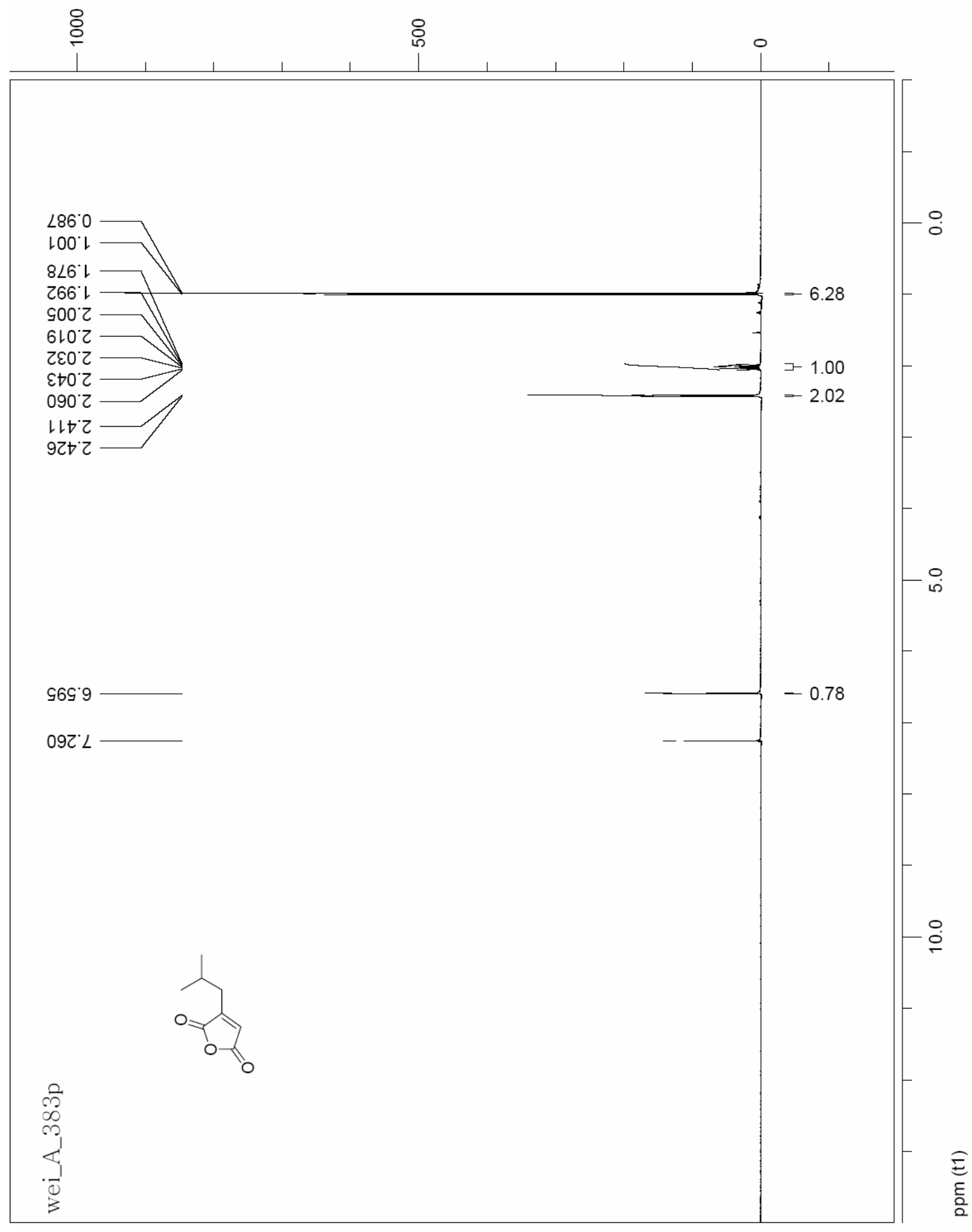




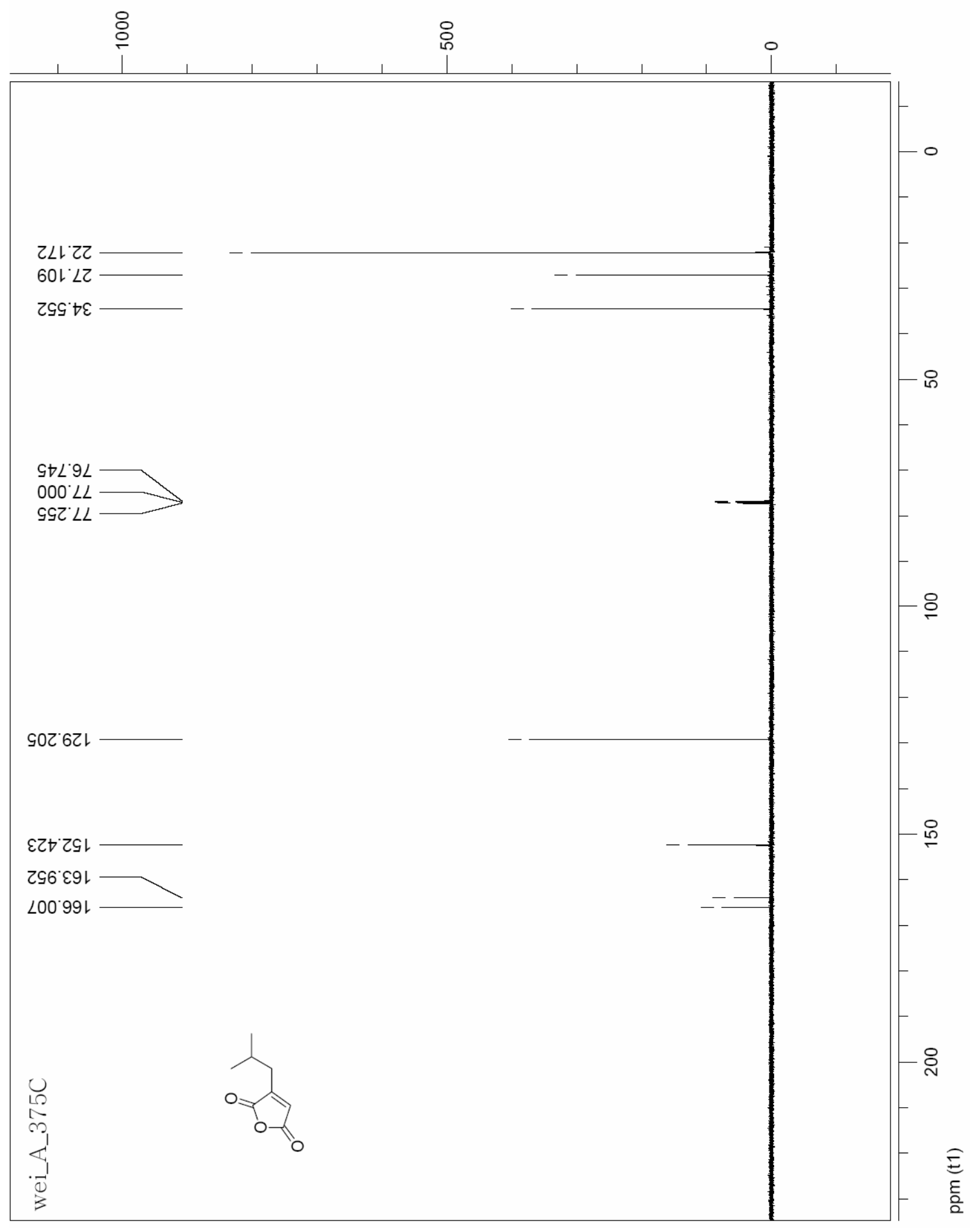




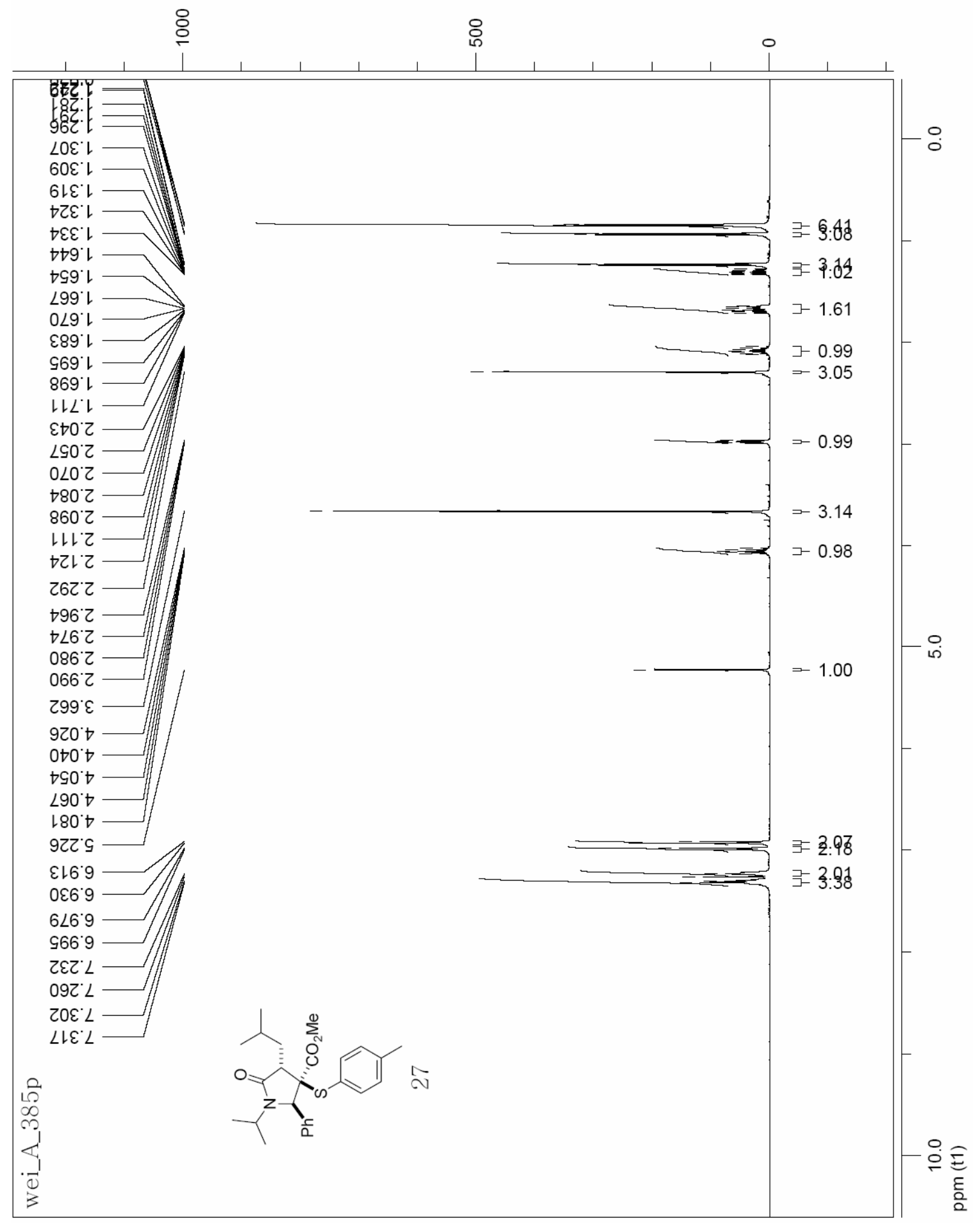




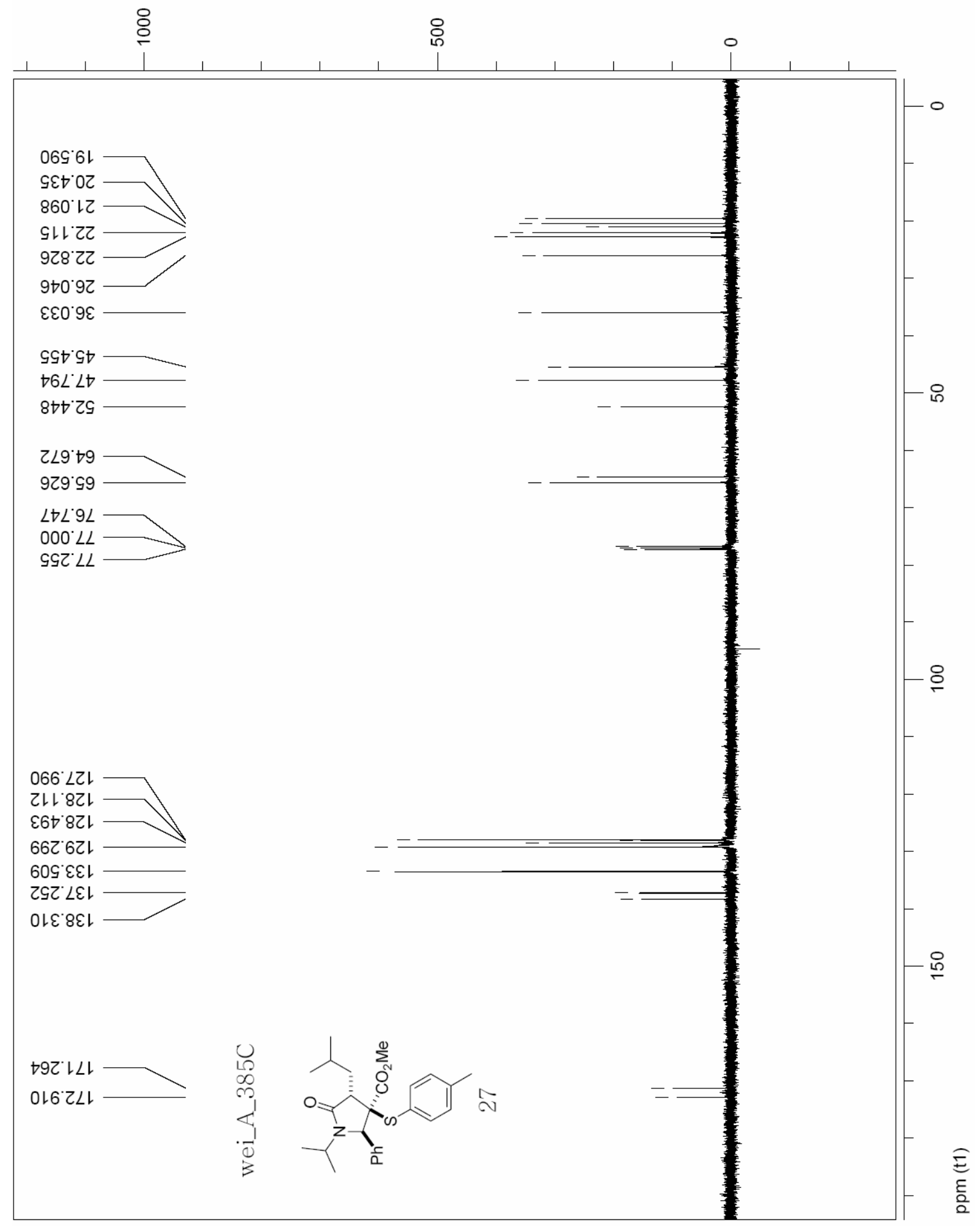




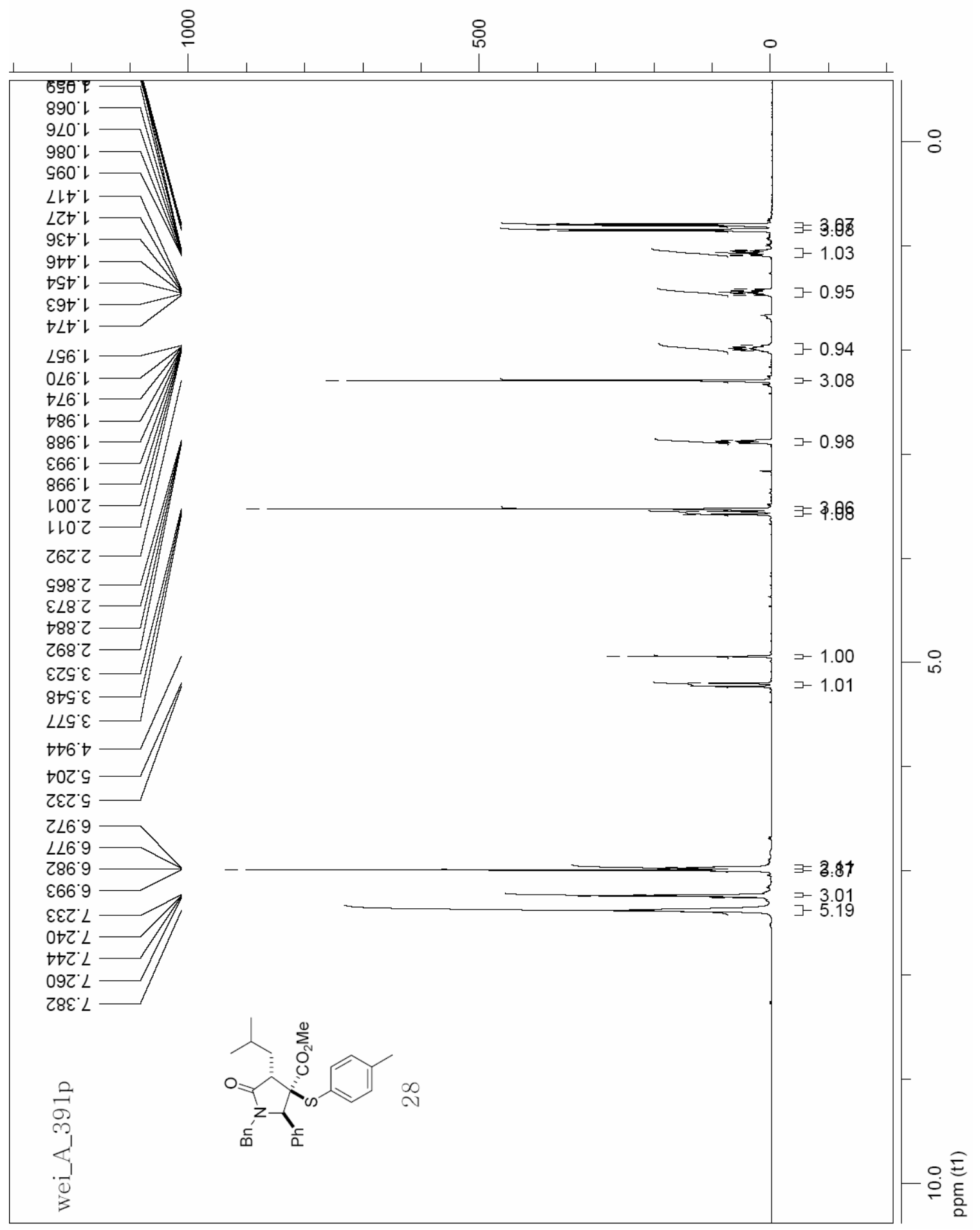




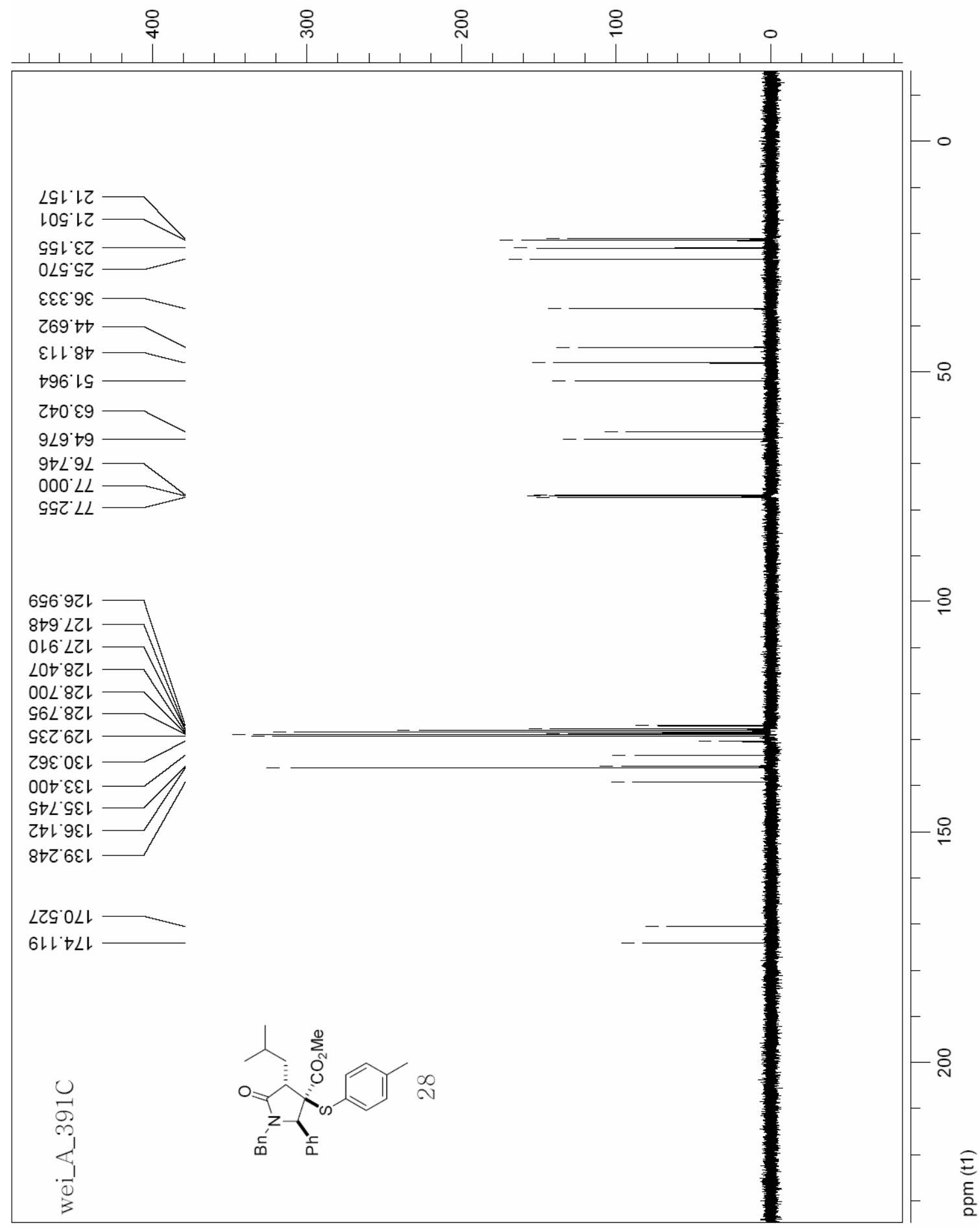




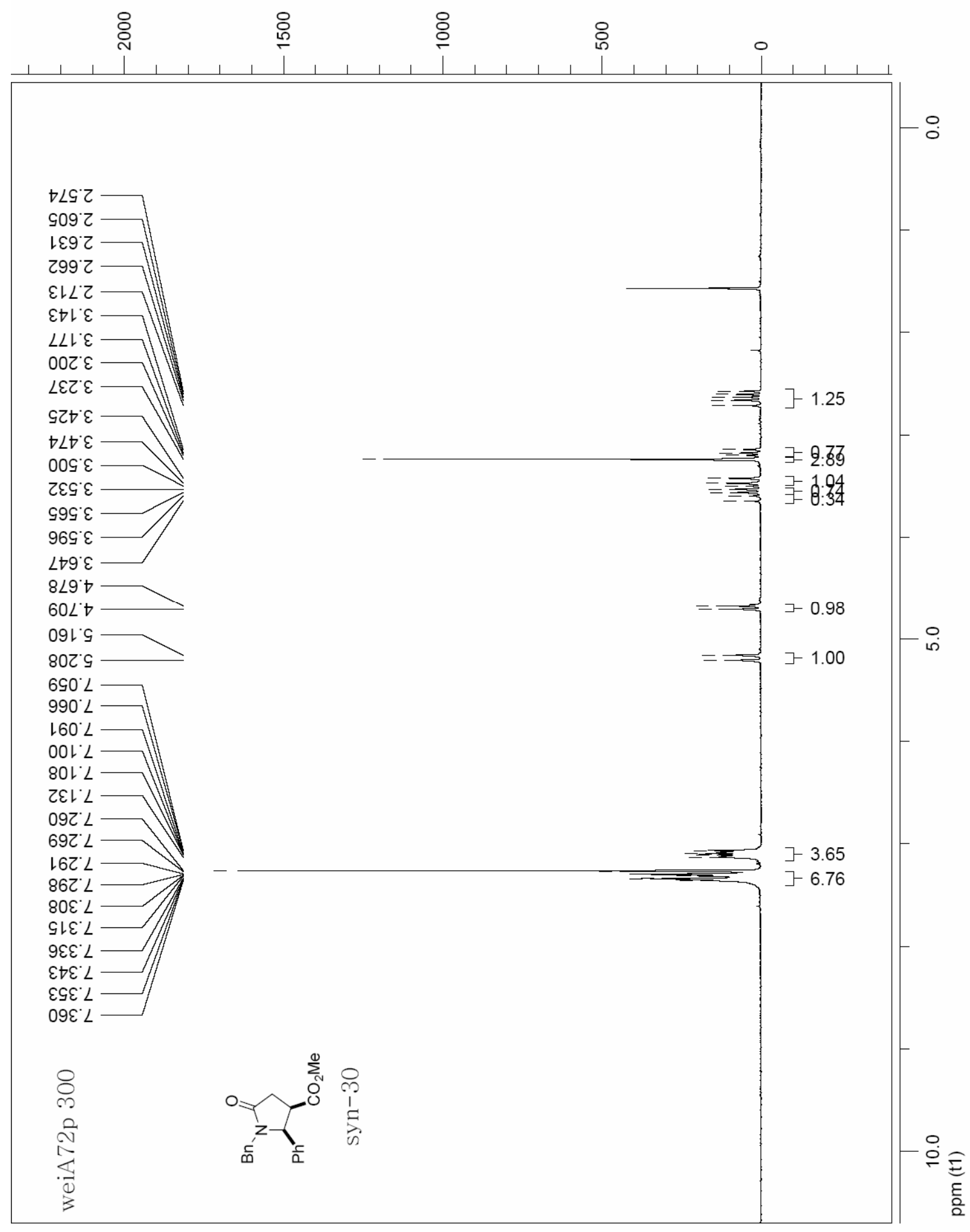




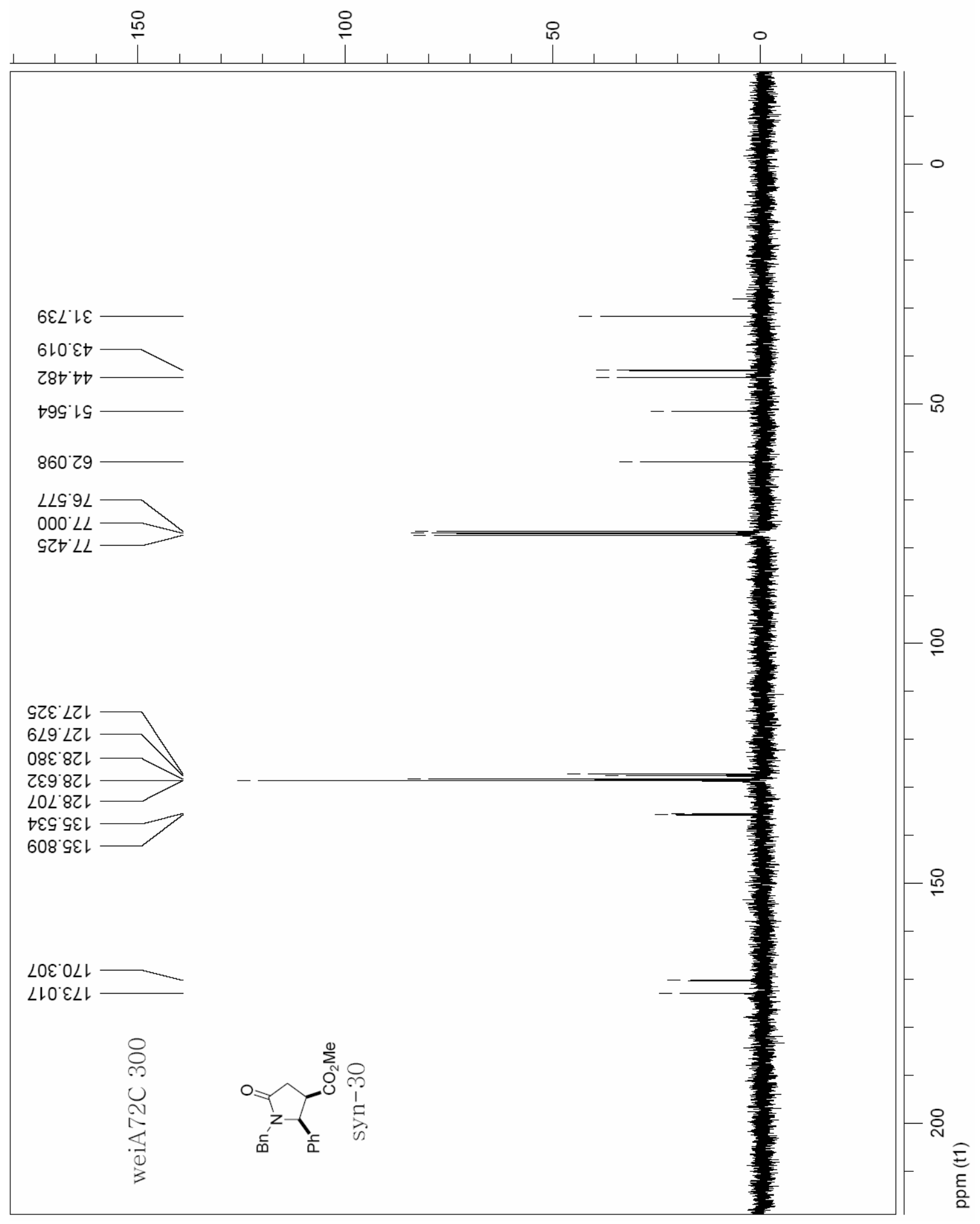




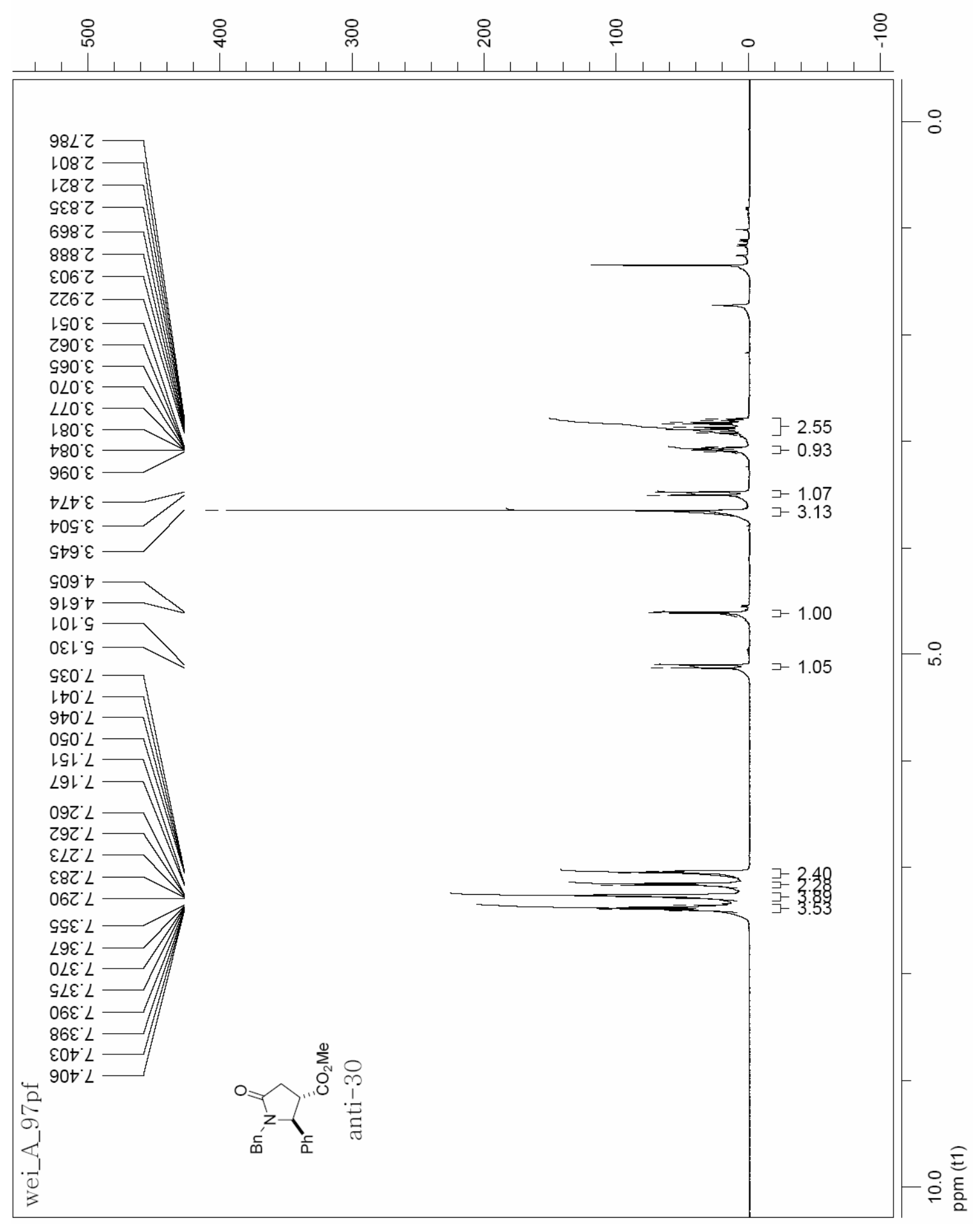




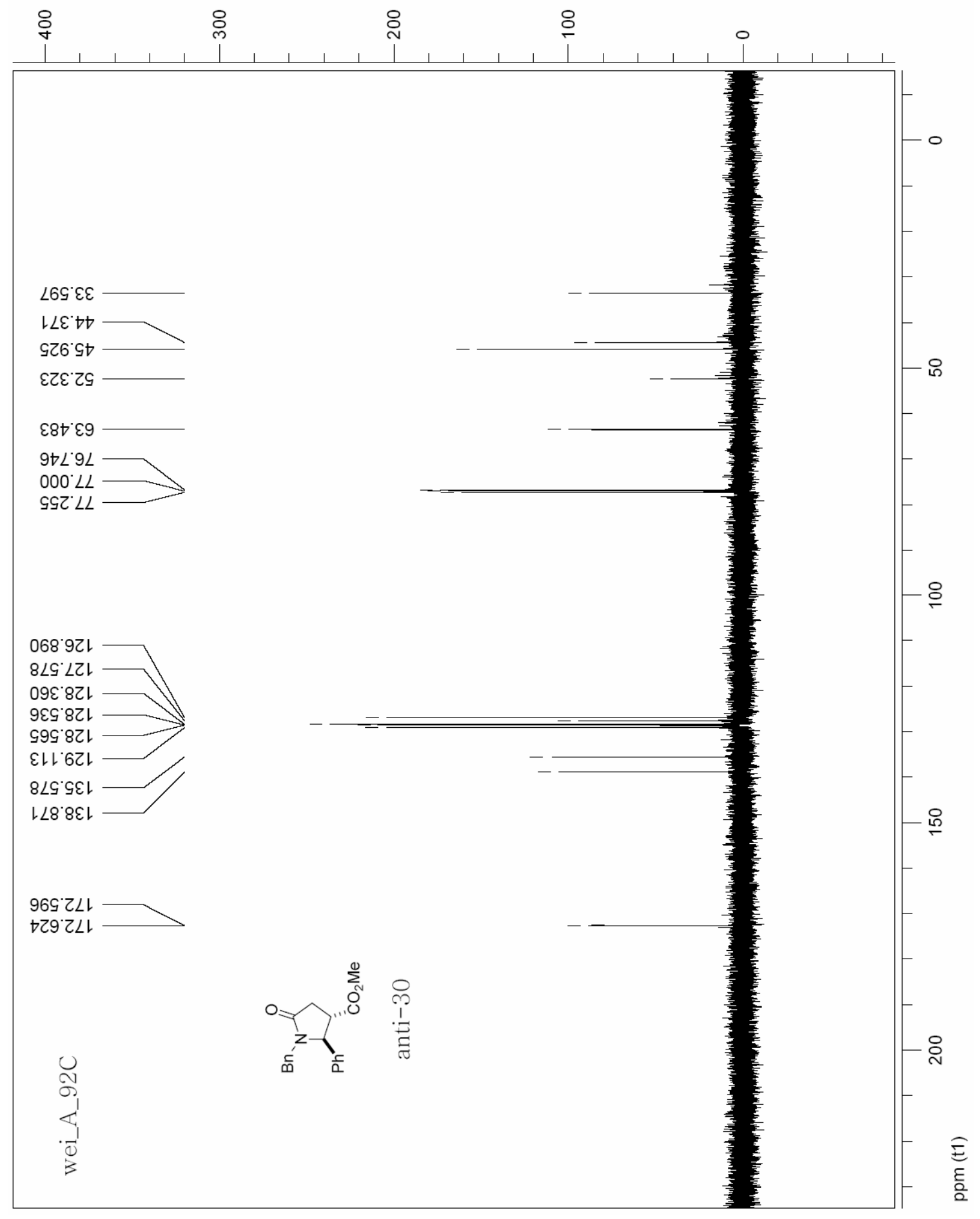


Date: May 8, 2007

Submitter: Jingqiang wei

Sample Reference Number: wei374

X-ray Number: JTS11
Center for Crystallographic Studies

Harvard University

Cambridge, MA 02138

Dr. Jingwei Huang

Dr. Richard J. Staples

Staples@chemistry.harvard.edu

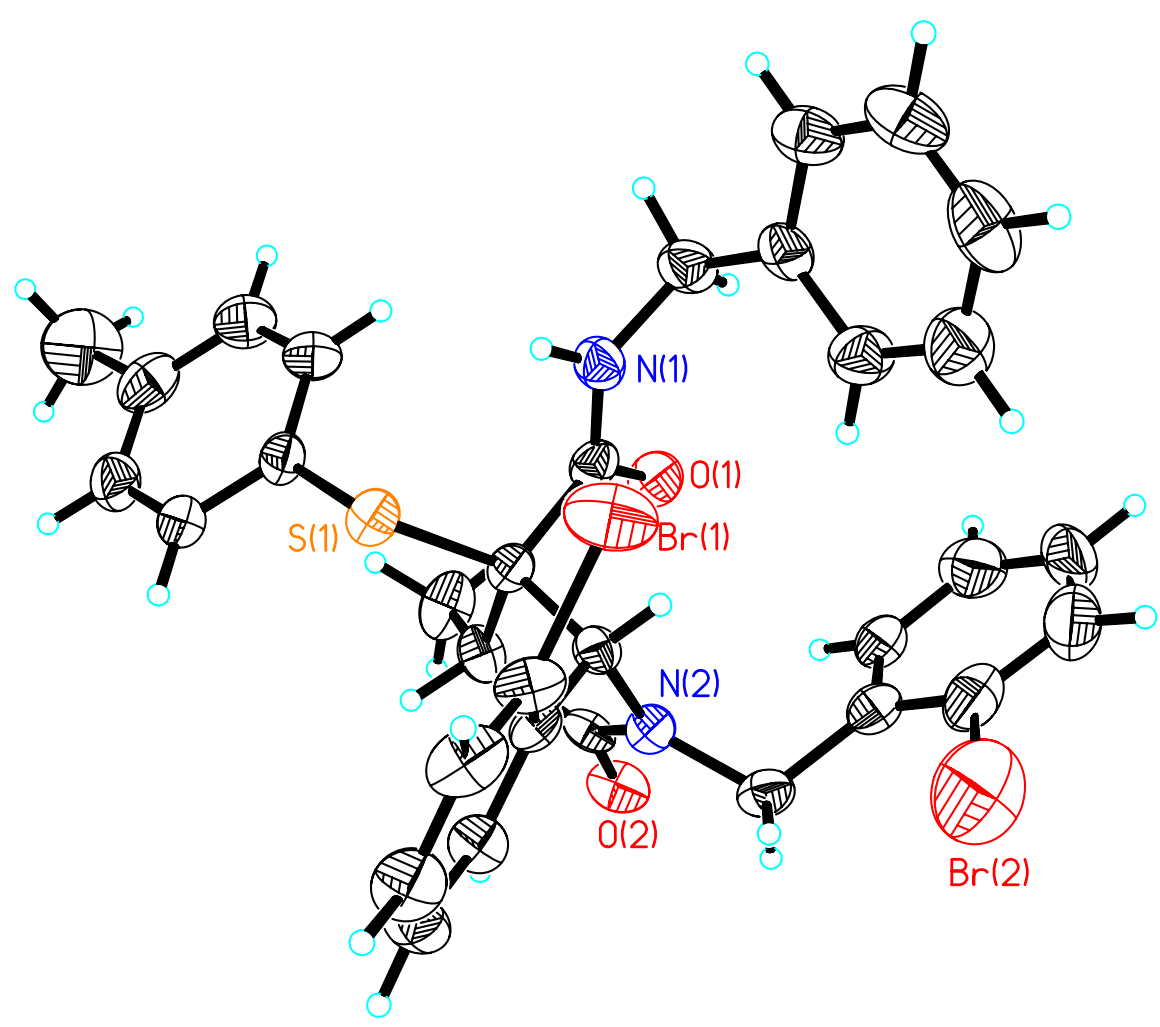

\section{Introduction:}

Single crystal study to confirm the identity of the sample submitted. 
Experimental Section:

A colorless block crystal with dimensions 0.25 x 0.05 x $0.04 \mathrm{~mm}$ was mounted on a $0.2 \mathrm{~nm}$ nylon loop using very small amount of paratone oil.

Data were collected using a Bruker SMART CCD (charge coupled device) based diffractometer equipped with an Oxford Cryostream low-temperature apparatus operating

at $193 \mathrm{~K}$. A suitable crystal was chosen and mounted on a glass fiber using grease. Data were measured using omega scans of $0.3^{\circ}$ per frame for 10 seconds, such that a hemisphere was collected. A total of 1800 frames were collected with a maximum resolution of $0.76 \AA$. The first 50 frames were recollected at the end of data collection to monitor for decay. Cell parameters were retrieved using SMART ${ }^{1}$ software and refined using SAINT on all observed reflections. Data reduction was performed using the SAINT software ${ }^{2}$ which corrects for Lp and decay. The structures are solved by the direct method using the SHELXS- $97^{3}$ program and refined by least squares method on $\mathrm{F}^{2}$, SHELXL-97, ${ }^{4}$ incorporated in SHELXTL-PC V $6.10 .^{5}$

The structure was solved in the space group P -1 (\# 2). All non-hydrogen atoms are refined anisotropically. Hydrogens were calculated by geometrical methods and refined as a riding model. The crystal used for the diffraction study showed no decomposition during data collection. All drawings are done at 50\% ellipsoids.

Acknowledgement. The CCD based x-ray diffractometer at Harvard University was purchased through NIH grant (1S10RR11937-01).

\section{References}

1. SMART V 5.625 (NT) Software for the CCD Detector System; Bruker Analytical X-ray Systems, Madison, WI (2001). 
2. SAINT V 6.22 (NT) Software for the CCD Detector System Bruker Analytical Xray Systems, Madison, WI (2001).

3. Sheldrick, G. M. SHELXS-90, Program for the Solution of Crystal Structure, University of Göttingen, Germany, 1990.

4. Sheldrick, G. M. SHELXL-97, Program for the Refinement of Crystal Structure, University of Göttingen, Germany, 1997.

5. SHELXTL 6.1 (PC-Version), Program library for Structure Solution and Molecular Graphics; Bruker Analytical X-ray Systems, Madison, WI (2000).

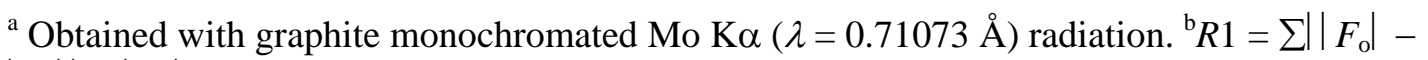
$\left|F_{\mathrm{c}}\right|\left|/ \sum\right| F_{\mathrm{o}} \mid .{ }^{\mathrm{c}} w R_{2}=\left\{\sum\left[w\left(F_{\mathrm{o}}{ }^{2}-F_{\mathrm{c}}{ }^{2}\right)^{2} /\left\{\sum\left[w\left(F_{\mathrm{o}}{ }^{2}\right)^{2}\right]\right\}^{1 / 2}\right.\right.$.

The following are 50\% thermal ellipsoidal drawings of the molecule in the asymmetric cell with various amount of labeling.

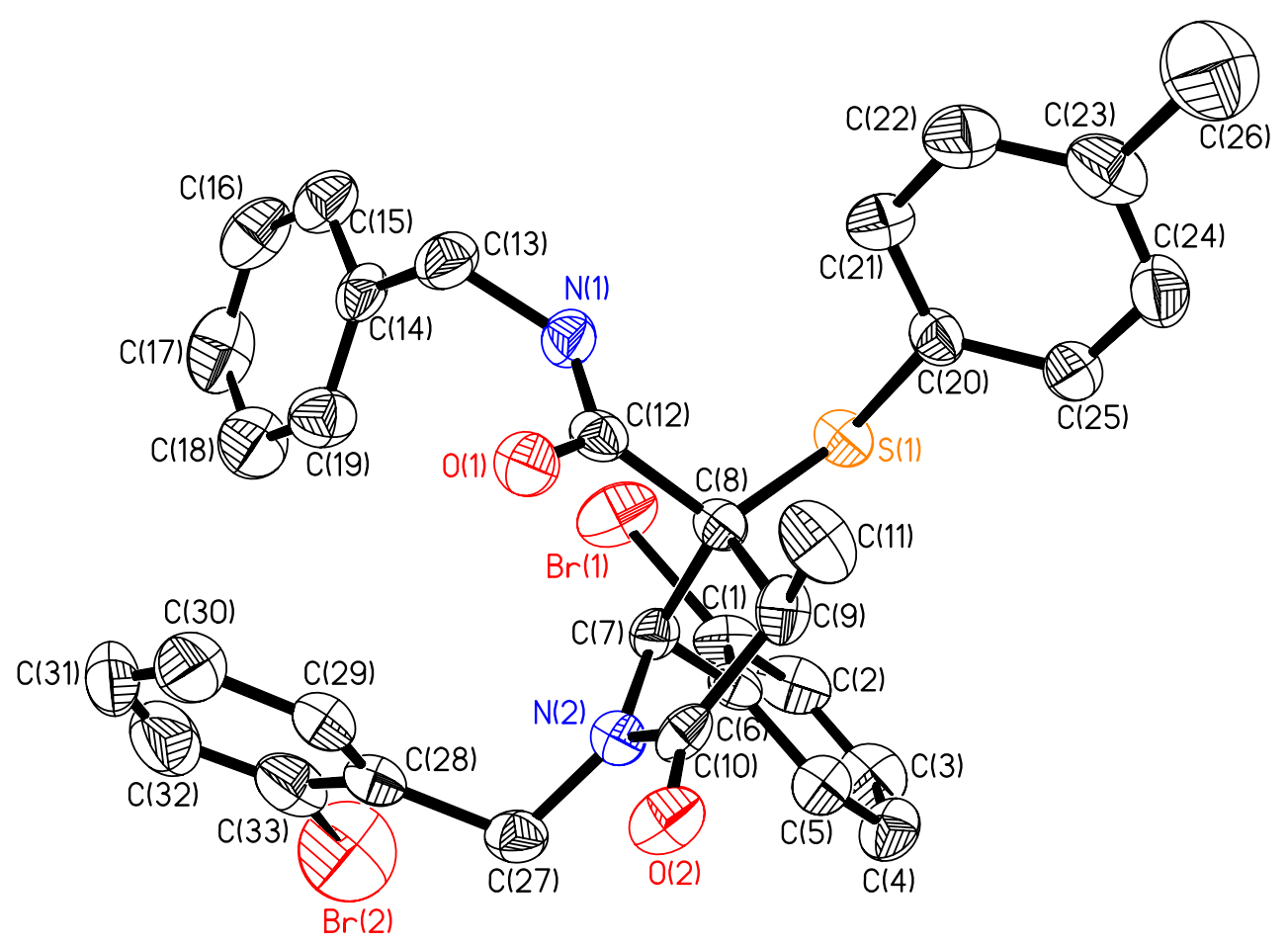




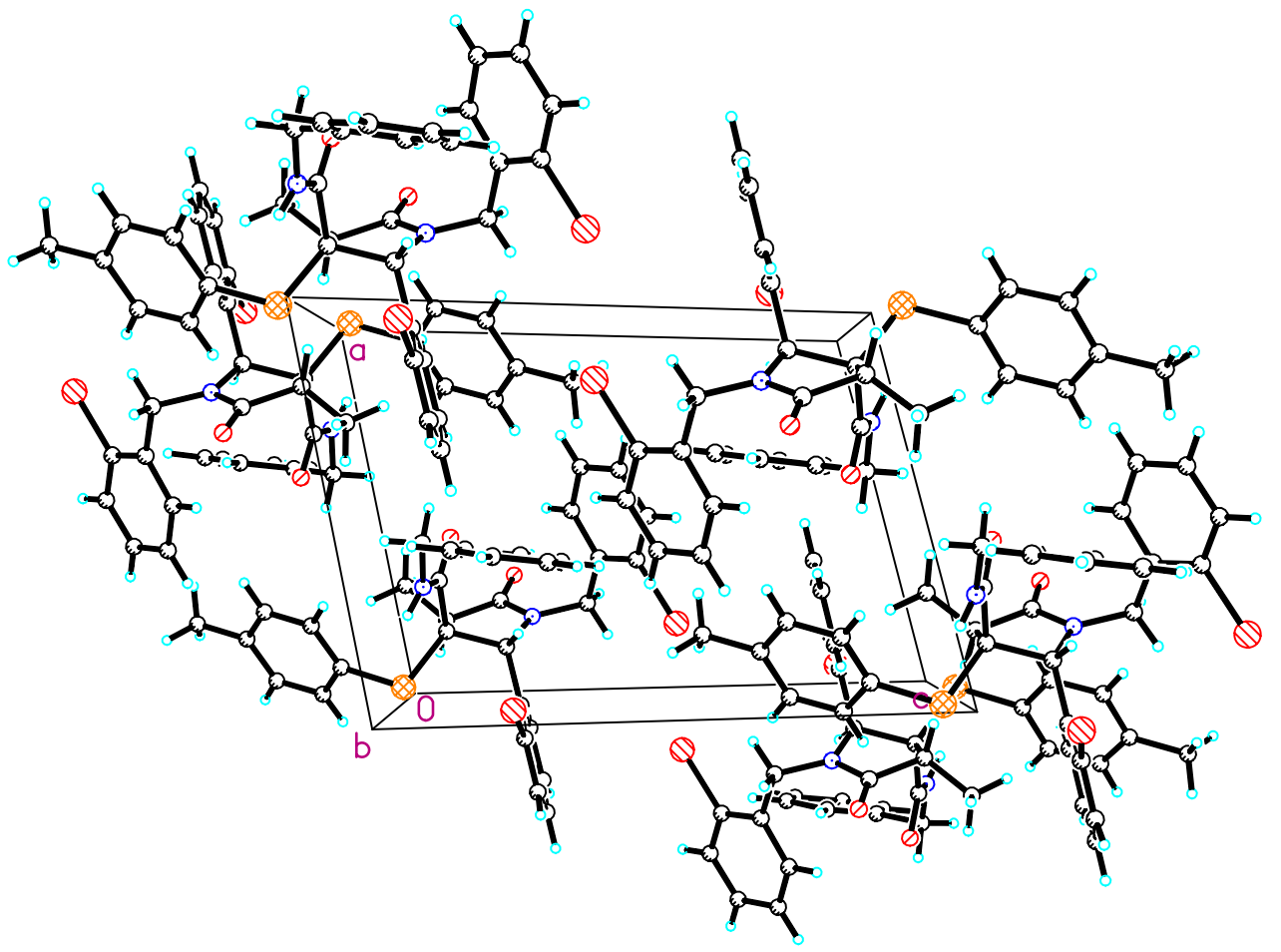

The last picture is the packing diagram along the b-axis. 
Table 1. Crystal data and structure refinement for jts11.

Identification code

Empirical formula

Formula weight

Temperature

Wavelength

Crystal system

Space group

Unit cell dimensions

Volume

Z

Density (calculated)

Absorption coefficient

$\mathrm{F}(000)$

Crystal size

Theta range for data collection

Index ranges

Reflections collected

Independent reflections

Completeness to theta $=25.00^{\circ}$

Absorption correction

Max. and min. transmission

Refinement method

Data / restraints / parameters

Goodness-of-fit on $\mathrm{F}^{2}$

Final R indices [I $>2 \operatorname{sigma}(\mathrm{I})]$

$\mathrm{R}$ indices (all data)

Largest diff. peak and hole jts11

C33 H30 Br2 N2 O2 S

678.47

193(2) K

$0.71073 \AA$

Triclinic

P-1

$\mathrm{a}=9.8222(17) \AA \quad \alpha=113.965(2)^{\circ}$.

$\mathrm{b}=12.934(2) \AA \quad \beta=93.434(3)^{\circ}$.

$\mathrm{c}=14.118(3) \AA \quad \gamma=109.602(3)^{\circ}$.

1503.3(5) $\AA^{3}$

2

$1.499 \mathrm{Mg} / \mathrm{m}^{3}$

$2.798 \mathrm{~mm}^{-1}$

688

$0.25 \times 0.05 \times 0.04 \mathrm{~mm}^{3}$

1.62 to $25.00^{\circ}$.

$-10<=\mathrm{h}<=11,-15<=\mathrm{k}<=15,-16<=\mathrm{l}<=16$

11437

$5270[\mathrm{R}(\mathrm{int})=0.0660]$

$99.6 \%$

None

0.8963 and 0.5413

Full-matrix least-squares on $\mathrm{F}^{2}$

5270 / 0 / 375

1.089

$\mathrm{R} 1=0.0693, \mathrm{wR} 2=0.1453$

$\mathrm{R} 1=0.0937, \mathrm{wR} 2=0.1562$

1.095 and -0.612 e. $\AA^{-3}$ 
Table 2. Atomic coordinates ( x $10^{4}$ ) and equivalent isotropic displacement parameters $\left(\AA^{2} \times 10^{3}\right.$ ) for jts11. $U(e q)$ is defined as one third of the trace of the orthogonalized $U^{i j}$ tensor.

\begin{tabular}{|c|c|c|c|c|}
\hline & $\mathrm{x}$ & $\mathrm{y}$ & $\mathrm{z}$ & $\mathrm{U}(\mathrm{eq})$ \\
\hline $\operatorname{Br}(1)$ & $-490(1)$ & 10314(1) & 1753(1) & $58(1)$ \\
\hline $\operatorname{Br}(2)$ & $1810(1)$ & $11160(1)$ & $5230(1)$ & $100(1)$ \\
\hline $\mathrm{C}(1)$ & $-1371(6)$ & 8772(5) & $1783(4)$ & $38(1)$ \\
\hline $\mathrm{C}(2)$ & $-2864(6)$ & $8366(6)$ & 1790(5) & $47(2)$ \\
\hline C(3) & $-3545(6)$ & 7269(6) & $1826(5)$ & $49(2)$ \\
\hline C(4) & $-2749(6)$ & $6590(5)$ & $1857(5)$ & $45(1)$ \\
\hline C(5) & $-1258(6)$ & 7009(5) & $1842(4)$ & $35(1)$ \\
\hline$C(6)$ & $-533(5)$ & 8119(4) & $1813(4)$ & $27(1)$ \\
\hline $\mathrm{C}(7)$ & 1111(6) & 8553(5) & 1798(4) & 28(1) \\
\hline$C(8)$ & $1534(5)$ & $8020(4)$ & $690(4)$ & $28(1)$ \\
\hline C(9) & 1792(6) & $6900(5)$ & 661(4) & $34(1)$ \\
\hline $\mathrm{C}(10)$ & 2344(5) & $7280(4)$ & $1817(4)$ & $30(1)$ \\
\hline $\mathrm{C}(11)$ & $2768(7)$ & 6382(6) & $-36(4)$ & $47(2)$ \\
\hline $\mathrm{C}(12)$ & 2985(6) & $9065(5)$ & 811(4) & $31(1)$ \\
\hline$C(13)$ & $4052(6)$ & 11057(5) & 774(5) & $41(1)$ \\
\hline$C(14)$ & $3889(5)$ & $12174(5)$ & 1596(4) & $36(1)$ \\
\hline$C(15)$ & 3891(6) & $13126(5)$ & 1389(5) & $47(2)$ \\
\hline$C(16)$ & $3739(7)$ & $14144(6)$ & $2157(6)$ & $57(2)$ \\
\hline $\mathrm{C}(17)$ & $3585(8)$ & $14210(6)$ & $3132(6)$ & $65(2)$ \\
\hline $\mathrm{C}(18)$ & $3570(7)$ & 13263(6) & $3354(5)$ & $59(2)$ \\
\hline C(19) & 3731(7) & 12260(5) & 2597(5) & $47(1)$ \\
\hline$C(20)$ & $522(5)$ & $6757(5)$ & $-1549(4)$ & $32(1)$ \\
\hline$C(21)$ & 1707(6) & 7302(5) & $-1903(4)$ & $39(1)$ \\
\hline$C(22)$ & 2078(7) & $6597(5)$ & $-2800(5)$ & $46(1)$ \\
\hline C(23) & $1268(7)$ & $5336(6)$ & $-3373(5)$ & $49(2)$ \\
\hline$C(24)$ & $52(7)$ & 4803(5) & $-3019(4)$ & $46(1)$ \\
\hline C(25) & $-299(6)$ & 5503(5) & $-2115(4)$ & $40(1)$ \\
\hline$C(26)$ & $1676(10)$ & $4555(7)$ & $-4350(6)$ & $81(2)$ \\
\hline$C(27)$ & 2297(6) & $8805(5)$ & $3565(4)$ & $36(1)$ \\
\hline$C(28)$ & $3597(6)$ & 10041(5) & 4012(4) & $40(1)$ \\
\hline C(29) & 4932(6) & 10098(5) & 3693(4) & $43(1)$ \\
\hline
\end{tabular}




$\begin{array}{lrrrr}\mathrm{C}(30) & 6146(8) & 11187(7) & 4075(6) & 61(2) \\ \mathrm{C}(31) & 6059(9) & 12252(6) & 4818(6) & 68(2) \\ \mathrm{C}(32) & 4783(10) & 12215(6) & 5162(5) & 70(2) \\ \mathrm{C}(33) & 3568(8) & 11128(6) & 4751(5) & 51(2) \\ \mathrm{N}(1) & 2836(5) & 9911(4) & 545(4) & 35(1) \\ \mathrm{N}(2) & 1923(4) & 8185(4) & 2409(3) & 29(1) \\ \mathrm{O}(1) & 4175(4) & 9148(3) & 1207(3) & 40(1) \\ \mathrm{O}(2) & 3051(4) & 6842(3) & 2180(3) & 39(1) \\ \mathrm{S}(1) & -2(1) & 7613(1) & -377(1) & 34(1) \\ \end{array}$

Table 3. Bond lengths $[\AA]$ and angles $\left[{ }^{\circ}\right]$ for jts 11 .

\begin{tabular}{|c|c|c|c|}
\hline $\operatorname{Br}(1)-C(1)$ & $1.908(5)$ & $\mathrm{C}(10)-\mathrm{O}(2)$ & $1.234(6)$ \\
\hline $\operatorname{Br}(2)-C(33)$ & $1.899(7)$ & $\mathrm{C}(10)-\mathrm{N}(2)$ & $1.346(6)$ \\
\hline$C(1)-C(6)$ & $1.373(7)$ & $\mathrm{C}(11)-\mathrm{H}(11 \mathrm{~A})$ & 0.9800 \\
\hline $\mathrm{C}(1)-\mathrm{C}(2)$ & $1.384(8)$ & $\mathrm{C}(11)-\mathrm{H}(11 \mathrm{~B})$ & 0.9800 \\
\hline$C(2)-C(3)$ & $1.374(9)$ & $\mathrm{C}(11)-\mathrm{H}(11 \mathrm{C})$ & 0.9800 \\
\hline $\mathrm{C}(2)-\mathrm{H}(2)$ & 0.9500 & $\mathrm{C}(12)-\mathrm{O}(1)$ & $1.216(6)$ \\
\hline$C(3)-C(4)$ & $1.369(8)$ & $\mathrm{C}(12)-\mathrm{N}(1)$ & $1.339(7)$ \\
\hline C(3)-H(3) & 0.9500 & $\mathrm{C}(13)-\mathrm{N}(1)$ & $1.451(7)$ \\
\hline$C(4)-C(5)$ & $1.385(7)$ & C(13)-C(14) & $1.509(7)$ \\
\hline $\mathrm{C}(4)-\mathrm{H}(4)$ & 0.9500 & $\mathrm{C}(13)-\mathrm{H}(13 \mathrm{~A})$ & 0.9900 \\
\hline$C(5)-C(6)$ & $1.390(7)$ & $\mathrm{C}(13)-\mathrm{H}(13 \mathrm{~B})$ & 0.9900 \\
\hline $\mathrm{C}(5)-\mathrm{H}(5)$ & 0.9500 & C(14)-C(15) & $1.375(8)$ \\
\hline $\mathrm{C}(6)-\mathrm{C}(7)$ & $1.527(7)$ & C(14)-C(19) & $1.394(8)$ \\
\hline $\mathrm{C}(7)-\mathrm{N}(2)$ & $1.453(6)$ & C(15)-C(16) & $1.387(9)$ \\
\hline$C(7)-C(8)$ & $1.577(7)$ & $\mathrm{C}(15)-\mathrm{H}(15)$ & 0.9500 \\
\hline $\mathrm{C}(7)-\mathrm{H}(7)$ & $0.89(4)$ & $\mathrm{C}(16)-\mathrm{C}(17)$ & $1.365(10)$ \\
\hline C(8)-C(9) & $1.538(7)$ & $\mathrm{C}(16)-\mathrm{H}(16)$ & 0.9500 \\
\hline $\mathrm{C}(8)-\mathrm{C}(12)$ & $1.542(7)$ & $\mathrm{C}(17)-\mathrm{C}(18)$ & $1.378(10)$ \\
\hline$C(8)-S(1)$ & $1.837(5)$ & $\mathrm{C}(17)-\mathrm{H}(17)$ & 0.9500 \\
\hline C(9)-C(10) & $1.505(7)$ & C(18)-C(19) & $1.371(8)$ \\
\hline C(9)-C(11) & $1.531(7)$ & $\mathrm{C}(18)-\mathrm{H}(18)$ & 0.9500 \\
\hline C(9)-H(9) & $0.97(5)$ & C(19)-H(19) & 0.9500 \\
\hline
\end{tabular}




\begin{tabular}{|c|c|c|c|}
\hline C(20)-C(21) & $1.380(7)$ & $\mathrm{C}(27)-\mathrm{N}(2)$ & $1.452(6)$ \\
\hline C(20)-C(25) & $1.383(7)$ & C(27)-C(28) & $1.516(8)$ \\
\hline$C(20)-S(1)$ & $1.796(5)$ & $\mathrm{C}(27)-\mathrm{H}(27 \mathrm{~A})$ & 0.9900 \\
\hline C(21)-C(22) & $1.381(8)$ & $\mathrm{C}(27)-\mathrm{H}(27 \mathrm{~B})$ & 0.9900 \\
\hline $\mathrm{C}(21)-\mathrm{H}(21)$ & 0.9500 & C(28)-C(33) & $1.380(8)$ \\
\hline C(22)-C(23) & 1.388(8) & C(28)-C(29) & $1.400(8)$ \\
\hline $\mathrm{C}(22)-\mathrm{H}(22)$ & 0.9500 & C(29)-C(30) & $1.370(9)$ \\
\hline C(23)-C(24) & $1.396(8)$ & C(29)-H(29) & 0.9500 \\
\hline C(23)-C(26) & $1.513(9)$ & C(30)-C(31) & $1.386(11)$ \\
\hline C(24)-C(25) & $1.375(8)$ & $\mathrm{C}(30)-\mathrm{H}(30)$ & 0.9500 \\
\hline C(24)-H(24) & 0.9500 & C(31)-C(32) & $1.365(11)$ \\
\hline C(25)-H(25) & 0.9500 & $\mathrm{C}(31)-\mathrm{H}(31)$ & 0.9500 \\
\hline $\mathrm{C}(26)-\mathrm{H}(26 \mathrm{~A})$ & 0.9800 & C(32)-C(33) & $1.366(10)$ \\
\hline $\mathrm{C}(26)-\mathrm{H}(26 \mathrm{~B})$ & 0.9800 & $\mathrm{C}(32)-\mathrm{H}(32)$ & 0.9500 \\
\hline $\mathrm{C}(26)-\mathrm{H}(26 \mathrm{C})$ & 0.9800 & $\mathrm{~N}(1)-\mathrm{H}(1 \mathrm{~N})$ & $0.81(6)$ \\
\hline $\mathrm{C}(6)-\mathrm{C}(1)-\mathrm{C}(2)$ & $122.4(5)$ & $\mathrm{C}(6)-\mathrm{C}(7)-\mathrm{C}(8)$ & $117.6(4)$ \\
\hline $\mathrm{C}(6)-\mathrm{C}(1)-\mathrm{Br}(1)$ & $120.5(4)$ & $\mathrm{N}(2)-\mathrm{C}(7)-\mathrm{H}(7)$ & 106(3) \\
\hline $\mathrm{C}(2)-\mathrm{C}(1)-\mathrm{Br}(1)$ & 117.1(4) & $\mathrm{C}(6)-\mathrm{C}(7)-\mathrm{H}(7)$ & 108(3) \\
\hline $\mathrm{C}(3)-\mathrm{C}(2)-\mathrm{C}(1)$ & 119.4(5) & $\mathrm{C}(8)-\mathrm{C}(7)-\mathrm{H}(7)$ & 111(3) \\
\hline $\mathrm{C}(3)-\mathrm{C}(2)-\mathrm{H}(2)$ & 120.3 & C(9)-C(8)-C(12) & $112.4(4)$ \\
\hline $\mathrm{C}(1)-\mathrm{C}(2)-\mathrm{H}(2)$ & 120.3 & C(9)-C(8)-C(7) & $102.4(4)$ \\
\hline $\mathrm{C}(4)-\mathrm{C}(3)-\mathrm{C}(2)$ & 120.1(5) & C(12)-C(8)-C(7) & 103.6(4) \\
\hline $\mathrm{C}(4)-\mathrm{C}(3)-\mathrm{H}(3)$ & 120.0 & $\mathrm{C}(9)-\mathrm{C}(8)-\mathrm{S}(1)$ & $113.4(3)$ \\
\hline $\mathrm{C}(2)-\mathrm{C}(3)-\mathrm{H}(3)$ & 120.0 & C(12)-C(8)-S(1) & 114.5(3) \\
\hline C(3)-C(4)-C(5) & $119.6(5)$ & $\mathrm{C}(7)-\mathrm{C}(8)-\mathrm{S}(1)$ & 109.3(3) \\
\hline $\mathrm{C}(3)-\mathrm{C}(4)-\mathrm{H}(4)$ & 120.2 & $\mathrm{C}(10)-\mathrm{C}(9)-\mathrm{C}(11)$ & $112.2(4)$ \\
\hline $\mathrm{C}(5)-\mathrm{C}(4)-\mathrm{H}(4)$ & 120.2 & $\mathrm{C}(10)-\mathrm{C}(9)-\mathrm{C}(8)$ & 103.1(4) \\
\hline $\mathrm{C}(4)-\mathrm{C}(5)-\mathrm{C}(6)$ & 121.8(5) & C(11)-C(9)-C(8) & $120.8(5)$ \\
\hline $\mathrm{C}(4)-\mathrm{C}(5)-\mathrm{H}(5)$ & 119.1 & $\mathrm{C}(10)-\mathrm{C}(9)-\mathrm{H}(9)$ & 111(3) \\
\hline $\mathrm{C}(6)-\mathrm{C}(5)-\mathrm{H}(5)$ & 119.1 & $\mathrm{C}(11)-\mathrm{C}(9)-\mathrm{H}(9)$ & 101(3) \\
\hline$C(1)-C(6)-C(5)$ & $116.8(5)$ & $\mathrm{C}(8)-\mathrm{C}(9)-\mathrm{H}(9)$ & 109(3) \\
\hline$C(1)-C(6)-C(7)$ & $122.9(4)$ & $\mathrm{O}(2)-\mathrm{C}(10)-\mathrm{N}(2)$ & $124.8(5)$ \\
\hline$C(5)-C(6)-C(7)$ & $120.3(4)$ & $\mathrm{O}(2)-\mathrm{C}(10)-\mathrm{C}(9)$ & $126.8(4)$ \\
\hline $\mathrm{N}(2)-\mathrm{C}(7)-\mathrm{C}(6)$ & $111.9(4)$ & N(2)-C(10)-C(9) & $108.4(4)$ \\
\hline $\mathrm{N}(2)-\mathrm{C}(7)-\mathrm{C}(8)$ & $101.3(4)$ & $\mathrm{C}(9)-\mathrm{C}(11)-\mathrm{H}(11 \mathrm{~A})$ & 109.5 \\
\hline
\end{tabular}




\begin{tabular}{|c|c|c|c|}
\hline C(9)-C(11)-H(11B) & 109.5 & $\mathrm{C}(20)-\mathrm{C}(21)-\mathrm{H}(21)$ & 119.9 \\
\hline $\mathrm{H}(11 \mathrm{~A})-\mathrm{C}(11)-\mathrm{H}(11 \mathrm{~B})$ & 109.5 & $\mathrm{C}(22)-\mathrm{C}(21)-\mathrm{H}(21)$ & 119.9 \\
\hline C(9)-C(11)-H(11C) & 109.5 & $\mathrm{C}(21)-\mathrm{C}(22)-\mathrm{C}(23)$ & $121.3(5)$ \\
\hline $\mathrm{H}(11 \mathrm{~A})-\mathrm{C}(11)-\mathrm{H}(11 \mathrm{C})$ & 109.5 & $\mathrm{C}(21)-\mathrm{C}(22)-\mathrm{H}(22)$ & 119.3 \\
\hline $\mathrm{H}(11 \mathrm{~B})-\mathrm{C}(11)-\mathrm{H}(11 \mathrm{C})$ & 109.5 & $\mathrm{C}(23)-\mathrm{C}(22)-\mathrm{H}(22)$ & 119.3 \\
\hline $\mathrm{O}(1)-\mathrm{C}(12)-\mathrm{N}(1)$ & 123.1(5) & C(22)-C(23)-C(24) & $117.8(5)$ \\
\hline $\mathrm{O}(1)-\mathrm{C}(12)-\mathrm{C}(8)$ & $120.4(5)$ & C(22)-C(23)-C(26) & 121.7(6) \\
\hline N(1)-C(12)-C(8) & $116.2(4)$ & $C(24)-C(23)-C(26)$ & $120.6(6)$ \\
\hline N(1)-C(13)-C(14) & 113.1(4) & $C(25)-C(24)-C(23)$ & $120.8(5)$ \\
\hline $\mathrm{N}(1)-\mathrm{C}(13)-\mathrm{H}(13 \mathrm{~A})$ & 109.0 & $\mathrm{C}(25)-\mathrm{C}(24)-\mathrm{H}(24)$ & 119.6 \\
\hline $\mathrm{C}(14)-\mathrm{C}(13)-\mathrm{H}(13 \mathrm{~A})$ & 109.0 & $\mathrm{C}(23)-\mathrm{C}(24)-\mathrm{H}(24)$ & 119.6 \\
\hline N(1)-C(13)-H(13B) & 109.0 & C(24)-C(25)-C(20) & $120.8(5)$ \\
\hline C(14)-C(13)-H(13B) & 109.0 & $\mathrm{C}(24)-\mathrm{C}(25)-\mathrm{H}(25)$ & 119.6 \\
\hline H(13A)-C(13)-H(13B) & 107.8 & $\mathrm{C}(20)-\mathrm{C}(25)-\mathrm{H}(25)$ & 119.6 \\
\hline C(15)-C(14)-C(19) & 118.3(5) & $\mathrm{C}(23)-\mathrm{C}(26)-\mathrm{H}(26 \mathrm{~A})$ & 109.5 \\
\hline C(15)-C(14)-C(13) & $121.8(5)$ & C(23)-C(26)-H(26B) & 109.5 \\
\hline$C(19)-C(14)-C(13)$ & $119.9(5)$ & $\mathrm{H}(26 \mathrm{~A})-\mathrm{C}(26)-\mathrm{H}(26 \mathrm{~B})$ & 109.5 \\
\hline C(14)-C(15)-C(16) & $120.8(6)$ & $\mathrm{C}(23)-\mathrm{C}(26)-\mathrm{H}(26 \mathrm{C})$ & 109.5 \\
\hline $\mathrm{C}(14)-\mathrm{C}(15)-\mathrm{H}(15)$ & 119.6 & $\mathrm{H}(26 \mathrm{~A})-\mathrm{C}(26)-\mathrm{H}(26 \mathrm{C})$ & 109.5 \\
\hline C(16)-C(15)-H(15) & 119.6 & H(26B)-C(26)-H(26C) & 109.5 \\
\hline C(17)-C(16)-C(15) & $120.0(6)$ & N(2)-C(27)-C(28) & $112.3(4)$ \\
\hline C(17)-C(16)-H(16) & 120.0 & $\mathrm{~N}(2)-\mathrm{C}(27)-\mathrm{H}(27 \mathrm{~A})$ & 109.1 \\
\hline C(15)-C(16)-H(16) & 120.0 & $\mathrm{C}(28)-\mathrm{C}(27)-\mathrm{H}(27 \mathrm{~A})$ & 109.1 \\
\hline$C(16)-C(17)-C(18)$ & $120.2(6)$ & N(2)-C(27)-H(27B) & 109.1 \\
\hline C(16)-C(17)-H(17) & 119.9 & C(28)-C(27)-H(27B) & 109.1 \\
\hline $\mathrm{C}(18)-\mathrm{C}(17)-\mathrm{H}(17)$ & 119.9 & $\mathrm{H}(27 \mathrm{~A})-\mathrm{C}(27)-\mathrm{H}(27 \mathrm{~B})$ & 107.9 \\
\hline C(19)-C(18)-C(17) & 119.8(6) & C(33)-C(28)-C(29) & $117.0(5)$ \\
\hline C(19)-C(18)-H(18) & 120.1 & $\mathrm{C}(33)-\mathrm{C}(28)-\mathrm{C}(27)$ & $123.9(5)$ \\
\hline C(17)-C(18)-H(18) & 120.1 & C(29)-C(28)-C(27) & 119.1(5) \\
\hline C(18)-C(19)-C(14) & $120.9(6)$ & C(30)-C(29)-C(28) & 121.7(6) \\
\hline C(18)-C(19)-H(19) & 119.5 & C(30)-C(29)-H(29) & 119.1 \\
\hline C(14)-C(19)-H(19) & 119.5 & $\mathrm{C}(28)-\mathrm{C}(29)-\mathrm{H}(29)$ & 119.1 \\
\hline C(21)-C(20)-C(25) & 119.1(5) & C(29)-C(30)-C(31) & $118.9(7)$ \\
\hline$C(21)-C(20)-S(1)$ & $122.6(4)$ & C(29)-C(30)-H(30) & 120.5 \\
\hline C(25)-C(20)-S(1) & 118.3(4) & $\mathrm{C}(31)-\mathrm{C}(30)-\mathrm{H}(30)$ & 120.5 \\
\hline C(20)-C(21)-C(22) & 120.2(5) & C(32)-C(31)-C(30) & $120.5(7)$ \\
\hline
\end{tabular}




$\begin{array}{llll}\mathrm{C}(32)-\mathrm{C}(31)-\mathrm{H}(31) & 119.8 & \mathrm{C}(12)-\mathrm{N}(1)-\mathrm{C}(13) & 123.5(5) \\ \mathrm{C}(30)-\mathrm{C}(31)-\mathrm{H}(31) & 119.8 & \mathrm{C}(12)-\mathrm{N}(1)-\mathrm{H}(1 \mathrm{~N}) & 118(4) \\ \mathrm{C}(31)-\mathrm{C}(32)-\mathrm{C}(33) & 119.8(7) & \mathrm{C}(13)-\mathrm{N}(1)-\mathrm{H}(1 \mathrm{~N}) & 118(4) \\ \mathrm{C}(31)-\mathrm{C}(32)-\mathrm{H}(32) & 120.1 & \mathrm{C}(10)-\mathrm{N}(2)-\mathrm{C}(27) & 123.9(4) \\ \mathrm{C}(33)-\mathrm{C}(32)-\mathrm{H}(32) & 120.1 & \mathrm{C}(10)-\mathrm{N}(2)-\mathrm{C}(7) & 114.5(4) \\ \mathrm{C}(32)-\mathrm{C}(33)-\mathrm{C}(28) & 122.0(6) & \mathrm{C}(27)-\mathrm{N}(2)-\mathrm{C}(7) & 121.5(4) \\ \mathrm{C}(32)-\mathrm{C}(33)-\mathrm{Br}(2) & 117.4(5) & \mathrm{C}(20)-\mathrm{S}(1)-\mathrm{C}(8) & 102.2(2) \\ \mathrm{C}(28)-\mathrm{C}(33)-\mathrm{Br}(2) & 120.6(5) & & \end{array}$

Symmetry transformations used to generate equivalent atoms:

Table 4. Anisotropic displacement parameters $\left(\AA^{2} \times 10^{3}\right)$ for jts11. The anisotropic displacement factor exponent takes the form: $-2 \pi^{2}\left[h^{2} a^{* 2} U^{11}+\ldots+2 h k a^{*} b^{*} U^{12}\right]$

\begin{tabular}{|c|c|c|c|c|c|c|}
\hline & $\mathrm{U}^{11}$ & $\mathrm{U}^{22}$ & $\mathrm{U}^{33}$ & $\mathrm{U}^{23}$ & $\mathrm{U}^{13}$ & $\mathrm{U}^{12}$ \\
\hline $\operatorname{Br}(1)$ & $61(1)$ & $58(1)$ & $89(1)$ & $50(1)$ & $30(1)$ & $41(1)$ \\
\hline $\operatorname{Br}(2)$ & $118(1)$ & $95(1)$ & 91(1) & 23(1) & $52(1)$ & $66(1)$ \\
\hline $\mathrm{C}(1)$ & $42(3)$ & $48(3)$ & $43(3)$ & $27(3)$ & 21(3) & $28(3)$ \\
\hline$C(2)$ & 41(3) & $77(4)$ & $50(4)$ & $36(3)$ & $23(3)$ & $42(3)$ \\
\hline$C(3)$ & $31(3)$ & $71(4)$ & $50(4)$ & $26(3)$ & $23(3)$ & $27(3)$ \\
\hline$C(4)$ & $36(3)$ & $45(3)$ & $51(4)$ & 21(3) & 21(3) & $12(3)$ \\
\hline C(5) & 32(3) & $38(3)$ & $40(3)$ & $16(2)$ & $15(2)$ & $20(2)$ \\
\hline$C(6)$ & 31(3) & $35(3)$ & $22(2)$ & $12(2)$ & $12(2)$ & $19(2)$ \\
\hline $\mathrm{C}(7)$ & $35(3)$ & 21(3) & $29(3)$ & $10(2)$ & $8(2)$ & $12(2)$ \\
\hline$C(8)$ & 24(3) & $36(3)$ & $26(3)$ & $14(2)$ & $6(2)$ & $12(2)$ \\
\hline $\mathrm{C}(9)$ & 31(3) & $27(3)$ & $38(3)$ & $8(2)$ & $7(2)$ & $14(2)$ \\
\hline$C(10)$ & 21(3) & $27(3)$ & $43(3)$ & $19(2)$ & $10(2)$ & $7(2)$ \\
\hline $\mathrm{C}(11)$ & 49(4) & $57(4)$ & $38(3)$ & $12(3)$ & $10(3)$ & $34(3)$ \\
\hline $\mathrm{C}(12)$ & 30(3) & $40(3)$ & $25(3)$ & $14(2)$ & $11(2)$ & $14(2)$ \\
\hline$C(13)$ & 41(3) & $39(3)$ & $44(3)$ & $20(3)$ & 19(3) & $12(3)$ \\
\hline$C(14)$ & 23(3) & $35(3)$ & $43(3)$ & $16(3)$ & $11(2)$ & $6(2)$ \\
\hline$C(15)$ & $35(3)$ & $49(4)$ & $64(4)$ & $34(3)$ & $12(3)$ & $15(3)$ \\
\hline$C(16)$ & $48(4)$ & $47(4)$ & $89(5)$ & $39(4)$ & $20(4)$ & $23(3)$ \\
\hline $\mathrm{C}(17)$ & 63(5) & $39(4)$ & $79(5)$ & $8(3)$ & $24(4)$ & $26(3)$ \\
\hline
\end{tabular}




\begin{tabular}{lllllll}
$\mathrm{C}(18)$ & $64(4)$ & $55(4)$ & $54(4)$ & $17(3)$ & $21(3)$ & $26(3)$ \\
$\mathrm{C}(19)$ & $57(4)$ & $44(3)$ & $47(4)$ & $24(3)$ & $16(3)$ & $24(3)$ \\
$\mathrm{C}(20)$ & $30(3)$ & $35(3)$ & $27(3)$ & $12(2)$ & $3(2)$ & $12(2)$ \\
$\mathrm{C}(21)$ & $40(3)$ & $42(3)$ & $42(3)$ & $24(3)$ & $16(3)$ & $16(3)$ \\
$\mathrm{C}(22)$ & $47(4)$ & $53(4)$ & $46(3)$ & $27(3)$ & $21(3)$ & $22(3)$ \\
$\mathrm{C}(23)$ & $65(4)$ & $58(4)$ & $37(3)$ & $21(3)$ & $16(3)$ & $36(4)$ \\
$\mathrm{C}(24)$ & $57(4)$ & $32(3)$ & $37(3)$ & $10(3)$ & $1(3)$ & $14(3)$ \\
$\mathrm{C}(25)$ & $39(3)$ & $41(3)$ & $34(3)$ & $18(3)$ & $1(3)$ & $7(3)$ \\
$\mathrm{C}(26)$ & $108(7)$ & $67(5)$ & $76(5)$ & $24(4)$ & $43(5)$ & $50(5)$ \\
$\mathrm{C}(27)$ & $34(3)$ & $44(3)$ & $35(3)$ & $23(3)$ & $13(2)$ & $15(3)$ \\
$\mathrm{C}(28)$ & $46(3)$ & $45(3)$ & $33(3)$ & $23(3)$ & $2(3)$ & $15(3)$ \\
$\mathrm{C}(29)$ & $46(4)$ & $47(3)$ & $33(3)$ & $21(3)$ & $0(3)$ & $13(3)$ \\
$\mathrm{C}(30)$ & $48(4)$ & $71(5)$ & $63(4)$ & $42(4)$ & $-1(3)$ & $9(4)$ \\
$\mathrm{C}(31)$ & $68(5)$ & $46(4)$ & $58(4)$ & $24(4)$ & $-24(4)$ & $-9(4)$ \\
$\mathrm{C}(32)$ & $99(6)$ & $46(4)$ & $45(4)$ & $13(3)$ & $-5(4)$ & $20(4)$ \\
$\mathrm{C}(33)$ & $70(4)$ & $54(4)$ & $34(3)$ & $18(3)$ & $9(3)$ & $32(4)$ \\
$\mathrm{N}(1)$ & $31(3)$ & $34(3)$ & $35(3)$ & $14(2)$ & $8(2)$ & $9(2)$ \\
$\mathrm{N}(2)$ & $24(2)$ & $34(2)$ & $30(2)$ & $15(2)$ & $7(2)$ & $13(2)$ \\
$\mathrm{O}(1)$ & $24(2)$ & $55(2)$ & $41(2)$ & $24(2)$ & $7(2)$ & $12(2)$ \\
$\mathrm{O}(2)$ & $31(2)$ & $42(2)$ & $59(2)$ & $31(2)$ & $14(2)$ & $21(2)$ \\
$\mathrm{S}(1)$ & $28(1)$ & $43(1)$ & $32(1)$ & $16(1)$ & $9(1)$ & $17(1)$ \\
\hline & & & & & & \\
\hline
\end{tabular}


Table 5. Hydrogen coordinates ( x 104) and isotropic displacement parameters ( $\left.\AA^{2} \mathrm{x} 10^{3}\right)$ for jts11.

\begin{tabular}{|c|c|c|c|c|}
\hline & $\mathrm{x}$ & $\mathrm{y}$ & $\mathrm{z}$ & $\mathrm{U}(\mathrm{eq})$ \\
\hline $\mathrm{H}(2)$ & -3412 & 8841 & 1770 & 57 \\
\hline $\mathrm{H}(3)$ & -4570 & 6983 & 1830 & 59 \\
\hline $\mathrm{H}(4)$ & -3216 & 5835 & 1888 & 54 \\
\hline $\mathrm{H}(5)$ & -716 & 6526 & 1851 & 42 \\
\hline $\mathrm{H}(11 \mathrm{~A})$ & 3761 & 7030 & 163 & 71 \\
\hline $\mathrm{H}(11 \mathrm{~B})$ & 2326 & 6087 & -785 & 71 \\
\hline $\mathrm{H}(11 \mathrm{C})$ & 2842 & 5697 & 70 & 71 \\
\hline $\mathrm{H}(13 \mathrm{~A})$ & 4111 & 11136 & 108 & 50 \\
\hline $\mathrm{H}(13 \mathrm{~B})$ & 4994 & 11030 & 1031 & 50 \\
\hline $\mathrm{H}(15)$ & 3999 & 13085 & 712 & 56 \\
\hline $\mathrm{H}(16)$ & 3741 & 14795 & 2004 & 68 \\
\hline $\mathrm{H}(17)$ & 3489 & 14910 & 3659 & 78 \\
\hline $\mathrm{H}(18)$ & 3448 & 13304 & 4029 & 71 \\
\hline $\mathrm{H}(19)$ & 3736 & 11615 & 2757 & 56 \\
\hline $\mathrm{H}(21)$ & 2270 & 8165 & -1527 & 47 \\
\hline $\mathrm{H}(22)$ & 2903 & 6983 & -3029 & 55 \\
\hline $\mathrm{H}(24)$ & -540 & 3947 & -3408 & 55 \\
\hline $\mathrm{H}(25)$ & -1114 & 5120 & -1876 & 48 \\
\hline $\mathrm{H}(26 \mathrm{~A})$ & 1230 & 4583 & -4974 & 122 \\
\hline $\mathrm{H}(26 \mathrm{~B})$ & 1304 & 3703 & -4457 & 122 \\
\hline $\mathrm{H}(26 \mathrm{C})$ & 2758 & 4874 & -4251 & 122 \\
\hline $\mathrm{H}(27 \mathrm{~A})$ & 2543 & 8275 & 3831 & 43 \\
\hline $\mathrm{H}(27 \mathrm{~B})$ & 1422 & 8930 & 3824 & 43 \\
\hline $\mathrm{H}(29)$ & 4997 & 9363 & 3199 & 51 \\
\hline $\mathrm{H}(30)$ & 7034 & 11212 & 3834 & 74 \\
\hline $\mathrm{H}(31)$ & 6894 & 13013 & 5089 & 82 \\
\hline $\mathrm{H}(32)$ & 4739 & 12944 & 5686 & 84 \\
\hline $\mathrm{H}(7)$ & $1500(40)$ & $9370(40)$ & $2110(30)$ & $9(10)$ \\
\hline $\mathrm{H}(9)$ & $850(60)$ & $6190(50)$ & $360(40)$ & $35(14)$ \\
\hline $\mathrm{H}(1 \mathrm{~N})$ & $2070(70)$ & $9710(50)$ & $140(50)$ & $44(18)$ \\
\hline
\end{tabular}


Table 6. Torsion angles $\left[{ }^{\circ}\right]$ for jts11.

\begin{tabular}{|c|c|}
\hline $\mathrm{C}(6)-\mathrm{C}(1)-\mathrm{C}(2)-\mathrm{C}(3)$ & $-0.1(9)$ \\
\hline Br(1)-C(1)-C(2)-C(3) & $-179.1(4)$ \\
\hline $\mathrm{C}(1)-\mathrm{C}(2)-\mathrm{C}(3)-\mathrm{C}(4)$ & $0.1(9)$ \\
\hline C(2)-C(3)-C(4)-C(5) & $-0.6(9)$ \\
\hline$C(3)-C(4)-C(5)-C(6)$ & $1.0(8)$ \\
\hline $\mathrm{C}(2)-\mathrm{C}(1)-\mathrm{C}(6)-\mathrm{C}(5)$ & $0.5(8)$ \\
\hline $\operatorname{Br}(1)-C(1)-C(6)-C(5)$ & 179.5(4) \\
\hline$C(2)-C(1)-C(6)-C(7)$ & 179.6(5) \\
\hline Br(1)-C(1)-C(6)-C(7) & $-1.4(7)$ \\
\hline$C(4)-C(5)-C(6)-C(1)$ & $-1.0(8)$ \\
\hline$C(4)-C(5)-C(6)-C(7)$ & 179.9(5) \\
\hline $\mathrm{C}(1)-\mathrm{C}(6)-\mathrm{C}(7)-\mathrm{N}(2)$ & $147.2(5)$ \\
\hline $\mathrm{C}(5)-\mathrm{C}(6)-\mathrm{C}(7)-\mathrm{N}(2)$ & $-33.7(6)$ \\
\hline$C(1)-C(6)-C(7)-C(8)$ & $-96.2(6)$ \\
\hline C(5)-C(6)-C(7)-C(8) & 82.9(6) \\
\hline N(2)-C(7)-C(8)-C(9) & 29.3(5) \\
\hline $\mathrm{C}(6)-\mathrm{C}(7)-\mathrm{C}(8)-\mathrm{C}(9)$ & $-92.9(5)$ \\
\hline N(2)-C(7)-C(8)-C(12) & $-87.7(4)$ \\
\hline $\mathrm{C}(6)-\mathrm{C}(7)-\mathrm{C}(8)-\mathrm{C}(12)$ & $150.1(4)$ \\
\hline N(2)-C(7)-C(8)-S(1) & 149.8(3) \\
\hline $\mathrm{C}(6)-\mathrm{C}(7)-\mathrm{C}(8)-\mathrm{S}(1)$ & $27.6(5)$ \\
\hline $\mathrm{C}(12)-\mathrm{C}(8)-\mathrm{C}(9)-\mathrm{C}(10)$ & $80.1(5)$ \\
\hline C(7)-C(8)-C(9)-C(10) & $-30.4(5)$ \\
\hline S(1)-C(8)-C(9)-C(10) & $-148.1(3)$ \\
\hline $\mathrm{C}(12)-\mathrm{C}(8)-\mathrm{C}(9)-\mathrm{C}(11)$ & $-46.1(6)$ \\
\hline $\mathrm{C}(7)-\mathrm{C}(8)-\mathrm{C}(9)-\mathrm{C}(11)$ & $-156.7(5)$ \\
\hline$S(1)-C(8)-C(9)-C(11)$ & $85.7(5)$ \\
\hline $\mathrm{C}(11)-\mathrm{C}(9)-\mathrm{C}(10)-\mathrm{O}(2)$ & $-28.3(7)$ \\
\hline $\mathrm{C}(8)-\mathrm{C}(9)-\mathrm{C}(10)-\mathrm{O}(2)$ & $-159.9(5)$ \\
\hline $\mathrm{C}(11)-\mathrm{C}(9)-\mathrm{C}(10)-\mathrm{N}(2)$ & 152.3(4) \\
\hline $\mathrm{C}(8)-\mathrm{C}(9)-\mathrm{C}(10)-\mathrm{N}(2)$ & $20.8(5)$ \\
\hline $\mathrm{C}(9)-\mathrm{C}(8)-\mathrm{C}(12)-\mathrm{O}(1)$ & $-24.0(6)$ \\
\hline $\mathrm{C}(7)-\mathrm{C}(8)-\mathrm{C}(12)-\mathrm{O}(1)$ & $85.8(5)$ \\
\hline $\mathrm{S}(1)-\mathrm{C}(8)-\mathrm{C}(12)-\mathrm{O}(1)$ & $-155.2(4)$ \\
\hline
\end{tabular}




\begin{tabular}{|c|c|}
\hline $\mathrm{C}(9)-\mathrm{C}(8)-\mathrm{C}(12)-\mathrm{N}(1)$ & $161.7(4)$ \\
\hline $\mathrm{C}(7)-\mathrm{C}(8)-\mathrm{C}(12)-\mathrm{N}(1)$ & $-88.5(5)$ \\
\hline $\mathrm{S}(1)-\mathrm{C}(8)-\mathrm{C}(12)-\mathrm{N}(1)$ & $30.5(5)$ \\
\hline N(1)-C(13)-C(14)-C(15) & $-123.6(6)$ \\
\hline N(1)-C(13)-C(14)-C(19) & $56.5(7)$ \\
\hline $\mathrm{C}(19)-\mathrm{C}(14)-\mathrm{C}(15)-\mathrm{C}(16)$ & $-0.1(8)$ \\
\hline C(13)-C(14)-C(15)-C(16) & $180.0(5)$ \\
\hline $\mathrm{C}(14)-\mathrm{C}(15)-\mathrm{C}(16)-\mathrm{C}(17)$ & $0.1(9)$ \\
\hline C(15)-C(16)-C(17)-C(18) & $-0.5(10)$ \\
\hline C(16)-C(17)-C(18)-C(19) & $1.0(11)$ \\
\hline $\mathrm{C}(17)-\mathrm{C}(18)-\mathrm{C}(19)-\mathrm{C}(14)$ & $-1.0(10)$ \\
\hline C(15)-C(14)-C(19)-C(18) & $0.6(9)$ \\
\hline $\mathrm{C}(13)-\mathrm{C}(14)-\mathrm{C}(19)-\mathrm{C}(18)$ & $-179.5(6)$ \\
\hline $\mathrm{C}(25)-\mathrm{C}(20)-\mathrm{C}(21)-\mathrm{C}(22)$ & $-1.0(8)$ \\
\hline$S(1)-C(20)-C(21)-C(22)$ & 178.9(4) \\
\hline C(20)-C(21)-C(22)-C(23) & $0.7(9)$ \\
\hline $\mathrm{C}(21)-\mathrm{C}(22)-\mathrm{C}(23)-\mathrm{C}(24)$ & $0.7(9)$ \\
\hline C(21)-C(22)-C(23)-C(26) & $-179.4(6)$ \\
\hline $\mathrm{C}(22)-\mathrm{C}(23)-\mathrm{C}(24)-\mathrm{C}(25)$ & $-1.9(9)$ \\
\hline C(26)-C(23)-C(24)-C(25) & 178.2(6) \\
\hline C(23)-C(24)-C(25)-C(20) & $1.7(9)$ \\
\hline C(21)-C(20)-C(25)-C(24) & $-0.2(8)$ \\
\hline S(1)-C(20)-C(25)-C(24) & 179.9(4) \\
\hline N(2)-C(27)-C(28)-C(33) & 127.5(5) \\
\hline N(2)-C(27)-C(28)-C(29) & $-54.6(6)$ \\
\hline C(33)-C(28)-C(29)-C(30) & $-1.6(8)$ \\
\hline C(27)-C(28)-C(29)-C(30) & $-179.6(5)$ \\
\hline $\mathrm{C}(28)-\mathrm{C}(29)-\mathrm{C}(30)-\mathrm{C}(31)$ & $1.8(9)$ \\
\hline C(29)-C(30)-C(31)-C(32) & $-0.1(10)$ \\
\hline C(30)-C(31)-C(32)-C(33) & $-1.8(11)$ \\
\hline C(31)-C(32)-C(33)-C(28) & $2.0(10)$ \\
\hline $\mathrm{C}(31)-\mathrm{C}(32)-\mathrm{C}(33)-\mathrm{Br}(2)$ & $-177.7(5)$ \\
\hline C(29)-C(28)-C(33)-C(32) & $-0.3(9)$ \\
\hline C(27)-C(28)-C(33)-C(32) & $177.6(6)$ \\
\hline $\mathrm{C}(29)-\mathrm{C}(28)-\mathrm{C}(33)-\mathrm{Br}(2)$ & 179.4(4) \\
\hline C(27)-C(28)-C(33)-Br(2) & $-2.7(8)$ \\
\hline
\end{tabular}




$\begin{array}{lc}\mathrm{O}(1)-\mathrm{C}(12)-\mathrm{N}(1)-\mathrm{C}(13) & -4.8(8) \\ \mathrm{C}(8)-\mathrm{C}(12)-\mathrm{N}(1)-\mathrm{C}(13) & 169.3(4) \\ \mathrm{C}(14)-\mathrm{C}(13)-\mathrm{N}(1)-\mathrm{C}(12) & -111.7(6) \\ \mathrm{O}(2)-\mathrm{C}(10)-\mathrm{N}(2)-\mathrm{C}(27) & 2.5(7) \\ \mathrm{C}(9)-\mathrm{C}(10)-\mathrm{N}(2)-\mathrm{C}(27) & -178.2(4) \\ \mathrm{O}(2)-\mathrm{C}(10)-\mathrm{N}(2)-\mathrm{C}(7) & 179.5(4) \\ \mathrm{C}(9)-\mathrm{C}(10)-\mathrm{N}(2)-\mathrm{C}(7) & -1.2(6) \\ \mathrm{C}(28)-\mathrm{C}(27)-\mathrm{N}(2)-\mathrm{C}(10) & 99.8(6) \\ \mathrm{C}(28)-\mathrm{C}(27)-\mathrm{N}(2)-\mathrm{C}(7) & -77.0(6) \\ \mathrm{C}(6)-\mathrm{C}(7)-\mathrm{N}(2)-\mathrm{C}(10) & 107.8(5) \\ \mathrm{C}(8)-\mathrm{C}(7)-\mathrm{N}(2)-\mathrm{C}(10) & -18.3(5) \\ \mathrm{C}(6)-\mathrm{C}(7)-\mathrm{N}(2)-\mathrm{C}(27) & -75.2(5) \\ \mathrm{C}(8)-\mathrm{C}(7)-\mathrm{N}(2)-\mathrm{C}(27) & 158.7(4) \\ \mathrm{C}(21)-\mathrm{C}(20)-\mathrm{S}(1)-\mathrm{C}(8) & -69.9(5) \\ \mathrm{C}(25)-\mathrm{C}(20)-\mathrm{S}(1)-\mathrm{C}(8) & 110.0(4) \\ \mathrm{C}(9)-\mathrm{C}(8)-\mathrm{S}(1)-\mathrm{C}(20) & -55.3(4) \\ \mathrm{C}(12)-\mathrm{C}(8)-\mathrm{S}(1)-\mathrm{C}(20) & 75.5(4) \\ \mathrm{C}(7)-\mathrm{C}(8)-\mathrm{S}(1)-\mathrm{C}(20) & -168.8(3)\end{array}$

Symmetry transformations used to generate equivalent atoms: 
Structural Data

Date: September 19, 2006

Submitter: Wei

Center for Crystallographic Studies

Harvard University

Sample Reference Number: Wei-1

Cambridge, MA 02138

Dr. Richard J. Staples

X-ray Number: jts06

Staples@chemistry.harvard.edu

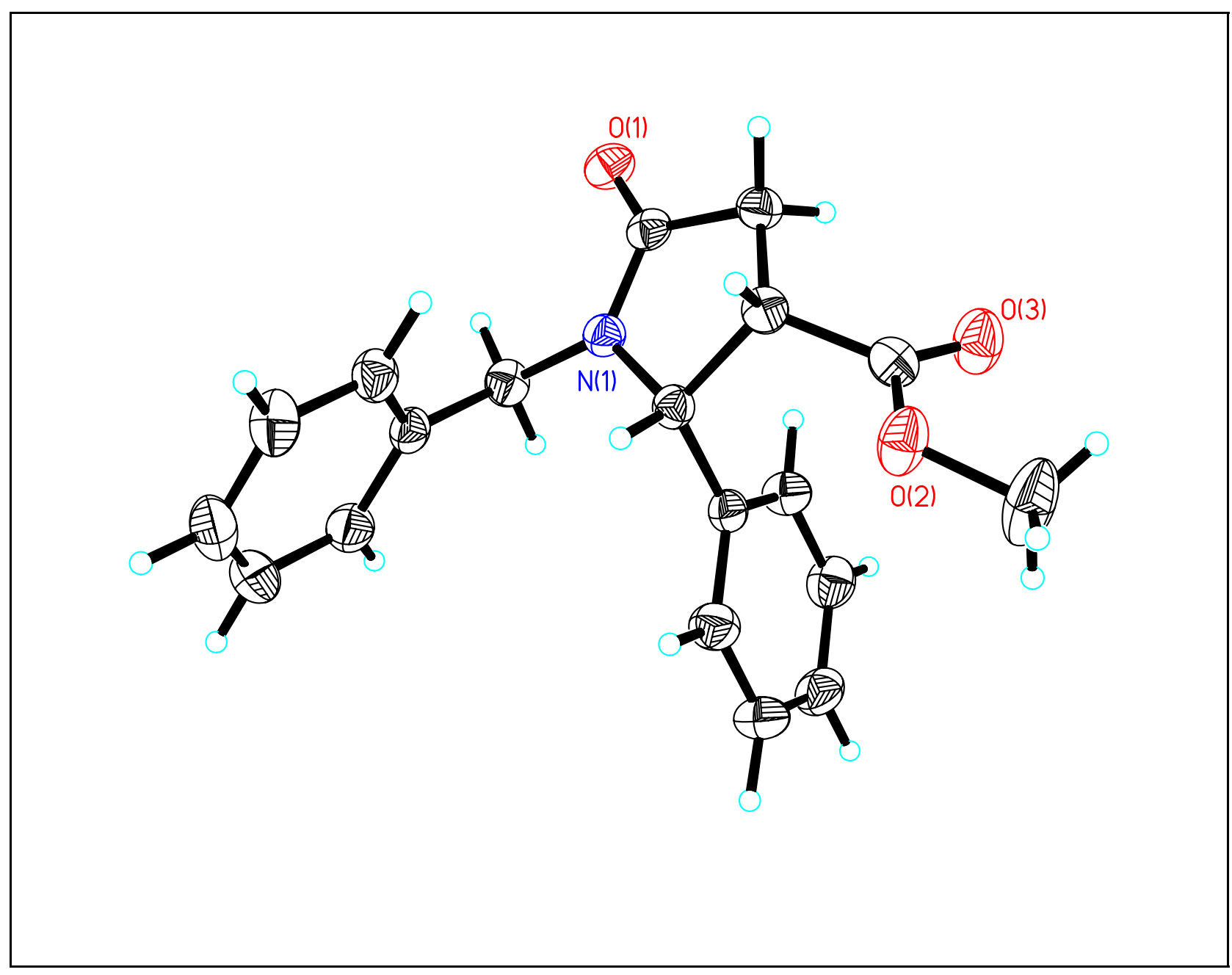

\section{Introduction:}

Single crystal study to confirm the identity of the sample submitted. 
Experimental Section:

A red block crystal with dimensions $0.10 \times 0.10 \times 0.10 \mathrm{~mm}$ was mounted on a 0.2 nm nylon loop using very small amount of paratone oil.

Data were collected using a Bruker SMART APEX CCD (charge coupled device) based diffractometer equipped with an Oxford Cryostream low-temperature apparatus operating at $193 \mathrm{~K}$. Data were measured using omega scans of $0.3^{\circ}$ per frame for 45 seconds, such that a hemisphere was collected. A total of 1271 frames were collected with a maximum resolution of $0.76 \AA$. The first 50 frames were recollected at the end of data collection to monitor for decay. Cell parameters were retrieved using SMART ${ }^{1}$ software and refined using SAINT on all observed reflections. Data reduction was performed using the SAINT software ${ }^{2}$ which corrects for Lp and decay. The structures are solved by the direct method using the SHELXS-97 ${ }^{3}$ program and refined by least squares method on $\mathrm{F}^{2}$, SHELXL-97, ${ }^{4}$ incorporated in SHELXTL-PC V 6.10. ${ }^{5}$

The structure was solved in the space group $\mathrm{P} 2{ }_{1} / \mathrm{n}$ (\# 14) by analysis of systematic absences. All non-hydrogen atoms are refined anisotropically. Hydrogens were found by difference Fourier methods and refined isotropically. The crystal used for the diffraction study showed no decomposition during data collection. All drawings are done at $50 \%$ ellipsoids.

Acknowledgement. The CCD based x-ray diffractometer at Harvard University was purchased through NIH grant (1S10RR11937-01). 


\section{References}

1. SMART V 5.625 (NT) Software for the CCD Detector System; Bruker Analytical X-ray Systems, Madison, WI (2001).

2. SAINT V 6.22 (NT) Software for the CCD Detector System Bruker Analytical Xray Systems, Madison, WI (2001).

3. Sheldrick, G. M. SHELXS-90, Program for the Solution of Crystal Structure, University of Göttingen, Germany, 1990.

4. Sheldrick, G. M. SHELXL-97, Program for the Refinement of Crystal Structure, University of Göttingen, Germany, 1997.

5. SHELXTL 6.1 (PC-Version), Program library for Structure Solution and Molecular Graphics; Bruker Analytical X-ray Systems, Madison, WI (2000) .

6. Flack, H. D.. Acta Cryst. A39, 1983, 876--881.

${ }^{\mathrm{a}}$ Obtained with graphite monochromated Mo K $\alpha(\lambda=0.71073 \AA)$ radiation. ${ }^{\mathrm{b}} R 1=\sum|| F_{\mathrm{o}} \mid-$ $\left|F_{\mathrm{c}}\right|\left|/ \sum\right| F_{\mathrm{o}} \mid .{ }^{\mathrm{c}} w R_{2}=\left\{\sum\left[w\left(F_{\mathrm{o}}{ }^{2}-F_{\mathrm{c}}{ }^{2}\right)^{2} /\left\{\sum\left[w\left(F_{\mathrm{o}}{ }^{2}\right)^{2}\right]\right\}^{1 / 2}\right.\right.$.

The following are 50\% thermal ellipsoidal drawings of the molecule in the asymmetric cell with various amount of labeling. 


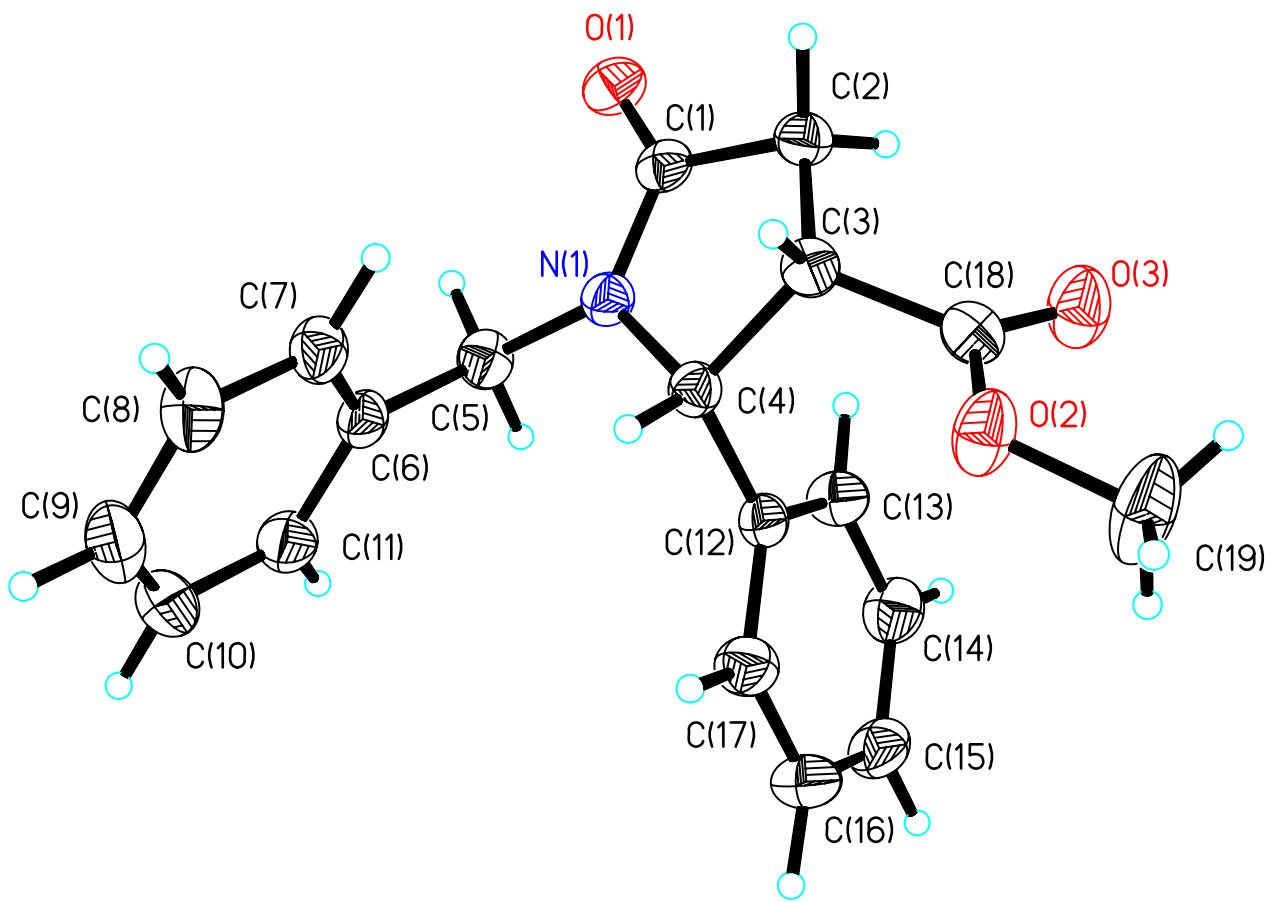




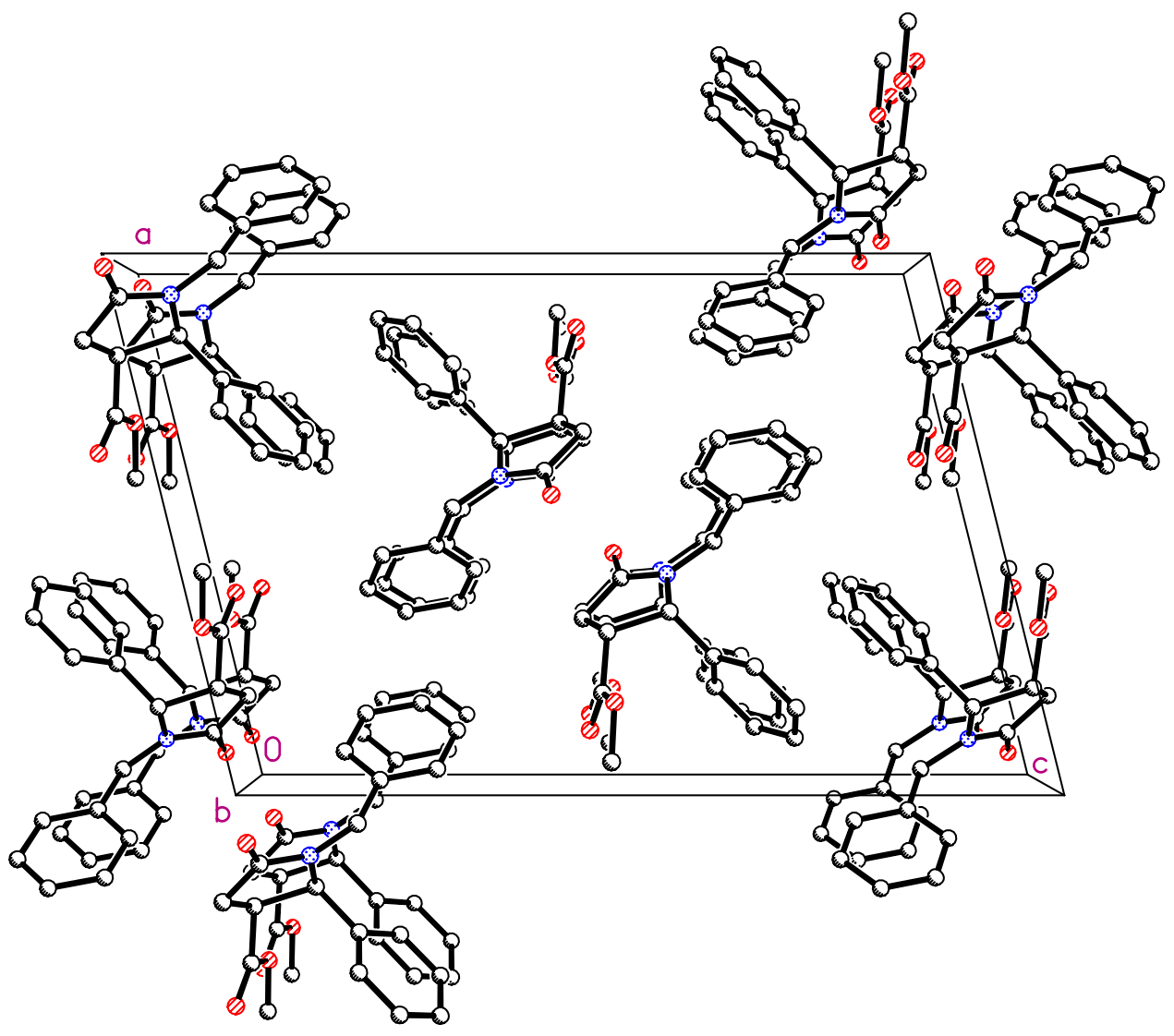

The last picture is the packing diagram along the b-axis. 
Table 1. Crystal data and structure refinement for jts06t.

\begin{tabular}{|c|c|c|}
\hline Identification code & \multicolumn{2}{|l|}{$\mathrm{jts} 06 \mathrm{t}$} \\
\hline Empirical formula & \multicolumn{2}{|l|}{ C19 H19 N O3 } \\
\hline Formula weight & \multicolumn{2}{|l|}{309.35} \\
\hline Temperature & \multicolumn{2}{|l|}{ 193(2) K } \\
\hline Wavelength & \multicolumn{2}{|l|}{$0.71073 \AA$} \\
\hline Crystal system & \multicolumn{2}{|l|}{ Monoclinic } \\
\hline Space group & \multicolumn{2}{|l|}{$\mathrm{P} 2(1) / \mathrm{n}$} \\
\hline \multirow[t]{3}{*}{ Unit cell dimensions } & $a=13.690(2) \AA$ & $\alpha=90^{\circ}$ \\
\hline & $\mathrm{b}=5.8634(8) \AA$ & $\beta=103.935(3)^{\circ}$. \\
\hline & $c=20.310(3) \AA$ & $\gamma=90^{\circ}$ \\
\hline Volume & \multicolumn{2}{|l|}{$1582.3(4) \AA^{3}$} \\
\hline $\mathrm{Z}$ & \multicolumn{2}{|l|}{4} \\
\hline Density (calculated) & \multicolumn{2}{|l|}{$1.299 \mathrm{Mg} / \mathrm{m}^{3}$} \\
\hline Absorption coefficient & \multicolumn{2}{|l|}{$0.088 \mathrm{~mm}^{-1}$} \\
\hline $\mathrm{F}(000)$ & \multicolumn{2}{|l|}{656} \\
\hline Crystal size & \multicolumn{2}{|c|}{$0.18 \times 0.16 \times 0.16 \mathrm{~mm}^{3}$} \\
\hline Theta range for data collection & \multicolumn{2}{|l|}{1.63 to $27.90^{\circ}$. } \\
\hline Index ranges & \multicolumn{2}{|c|}{$-10<=\mathrm{h}<=17,-7<=\mathrm{k}<=4,-26<=\mathrm{l}<=26$} \\
\hline Reflections collected & \multicolumn{2}{|l|}{4608} \\
\hline Independent reflections & \multicolumn{2}{|c|}{$3420[\mathrm{R}(\mathrm{int})=0.0329]$} \\
\hline Completeness to theta $=27.90^{\circ}$ & \multicolumn{2}{|l|}{$90.3 \%$} \\
\hline Absorption correction & \multicolumn{2}{|l|}{ None } \\
\hline Max. and min. transmission & \multicolumn{2}{|c|}{0.9861 and 0.9844} \\
\hline Refinement method & \multicolumn{2}{|c|}{ Full-matrix least-squares on $\mathrm{F}^{2}$} \\
\hline Data / restraints / parameters & \multicolumn{2}{|l|}{3420 / 0 / 284} \\
\hline Goodness-of-fit on $\mathrm{F}^{2}$ & \multicolumn{2}{|l|}{1.021} \\
\hline Final $\mathrm{R}$ indices [I>2sigma(I)] & \multicolumn{2}{|c|}{$\mathrm{R} 1=0.0390, \mathrm{wR} 2=0.0927$} \\
\hline R indices (all data) & \multicolumn{2}{|c|}{$\mathrm{R} 1=0.0619, \mathrm{wR} 2=0.1047$} \\
\hline Largest diff. peak and hole & \multicolumn{2}{|c|}{0.235 and -0.243 e. $\AA^{-3}$} \\
\hline
\end{tabular}


Table 2. Atomic coordinates ( $\times 10^{4}$ ) and equivalent isotropic displacement parameters $\left(\AA^{2} \times 10^{3}\right)$ for jts06t. $U(e q)$ is defined as one third of the trace of the orthogonalized $U^{i j}$ tensor.

\begin{tabular}{|c|c|c|c|c|}
\hline & $\mathrm{x}$ & $\mathrm{y}$ & $\mathrm{z}$ & $\mathrm{U}(\mathrm{eq})$ \\
\hline $\mathrm{O}(1)$ & $9462(1)$ & $7212(2)$ & $287(1)$ & $32(1)$ \\
\hline $\mathrm{O}(2)$ & 6882(1) & $-1021(2)$ & $-143(1)$ & $39(1)$ \\
\hline $\mathrm{O}(3)$ & $6286(1)$ & $2408(2)$ & $-518(1)$ & $43(1)$ \\
\hline $\mathrm{N}(1)$ & 9091(1) & 4083(2) & $858(1)$ & $25(1)$ \\
\hline $\mathrm{C}(1)$ & $9014(1)$ & $5409(2)$ & 301(1) & $25(1)$ \\
\hline $\mathrm{C}(2)$ & $8274(1)$ & 4309(3) & $-281(1)$ & $28(1)$ \\
\hline C(3) & $8065(1)$ & 1956(3) & $-26(1)$ & $26(1)$ \\
\hline C(4) & $8429(1)$ & $2100(2)$ & 767(1) & $24(1)$ \\
\hline$C(5)$ & 9693(1) & 4721(3) & 1524(1) & $29(1)$ \\
\hline$C(6)$ & 10393(1) & 2839(3) & 1863(1) & $27(1)$ \\
\hline$C(7)$ & 10859(1) & 1393(3) & 1489(1) & $32(1)$ \\
\hline C(8) & 11503(1) & $-306(3)$ & $1810(1)$ & $38(1)$ \\
\hline $\mathrm{C}(9)$ & 11682(1) & $-598(3)$ & 2506(1) & $42(1)$ \\
\hline$C(10)$ & $11224(1)$ & $829(3)$ & 2879(1) & $42(1)$ \\
\hline $\mathrm{C}(11)$ & 10582(1) & 2537(3) & 2561(1) & $35(1)$ \\
\hline $\mathrm{C}(12)$ & 7616(1) & $2345(2)$ & 1154(1) & $25(1)$ \\
\hline $\mathrm{C}(13)$ & 7023(1) & 4297(3) & 1099(1) & $32(1)$ \\
\hline$C(14)$ & $6282(1)$ & 4489(3) & $1457(1)$ & $37(1)$ \\
\hline$C(15)$ & $6137(1)$ & 2745(3) & 1882(1) & $38(1)$ \\
\hline$C(16)$ & $6728(1)$ & $819(3)$ & 1951(1) & $40(1)$ \\
\hline $\mathrm{C}(17)$ & 7465(1) & $618(3)$ & 1585(1) & $33(1)$ \\
\hline C(18) & 6984(1) & 1197(3) & $-261(1)$ & 28(1) \\
\hline C(19) & $5868(2)$ & $-1934(4)$ & $-343(2)$ & $55(1)$ \\
\hline
\end{tabular}


Table 3. Bond lengths $[\AA]$ and angles $\left[{ }^{\circ}\right]$ for jts06t.

\begin{tabular}{|c|c|c|c|}
\hline $\mathrm{O}(1)-\mathrm{C}(1)$ & $1.2260(17)$ & $\mathrm{O}(2)-\mathrm{C}(18)$ & $1.3356(18)$ \\
\hline $\mathrm{O}(2)-\mathrm{C}(19)$ & $1.451(2)$ & $\mathrm{O}(3)-\mathrm{C}(18)$ & $1.2046(18)$ \\
\hline $\mathrm{N}(1)-\mathrm{C}(1)$ & $1.3555(18)$ & $\mathrm{N}(1)-\mathrm{C}(5)$ & $1.4527(19)$ \\
\hline $\mathrm{N}(1)-\mathrm{C}(4)$ & $1.4589(18)$ & $\mathrm{C}(1)-\mathrm{C}(2)$ & $1.505(2)$ \\
\hline $\mathrm{C}(2)-\mathrm{C}(3)$ & $1.525(2)$ & $\mathrm{C}(2)-\mathrm{H}(2)$ & $0.970(16)$ \\
\hline $\mathrm{C}(2)-\mathrm{H}(1)$ & $0.984(16)$ & $C(3)-C(18)$ & $1.509(2)$ \\
\hline$C(3)-C(4)$ & $1.568(2)$ & $\mathrm{C}(3)-\mathrm{H}(3)$ & $0.940(15)$ \\
\hline$C(4)-C(12)$ & $1.5171(18)$ & $\mathrm{C}(4)-\mathrm{H}(4)$ & $0.982(14)$ \\
\hline$C(5)-C(6)$ & $1.514(2)$ & $\mathrm{C}(5)-\mathrm{H}(5)$ & $1.012(16)$ \\
\hline $\mathrm{C}(5)-\mathrm{H}(6)$ & $1.004(17)$ & $C(6)-C(11)$ & $1.391(2)$ \\
\hline$C(6)-C(7)$ & $1.391(2)$ & $C(7)-C(8)$ & $1.385(2)$ \\
\hline $\mathrm{C}(7)-\mathrm{H}(7)$ & $0.984(16)$ & $\mathrm{C}(8)-\mathrm{C}(9)$ & $1.386(3)$ \\
\hline $\mathrm{C}(8)-\mathrm{H}(8)$ & $0.977(18)$ & $\mathrm{C}(9)-\mathrm{C}(10)$ & $1.378(3)$ \\
\hline $\mathrm{C}(9)-\mathrm{H}(9)$ & $0.955(18)$ & $\mathrm{C}(10)-\mathrm{C}(11)$ & $1.385(2)$ \\
\hline $\mathrm{C}(10)-\mathrm{H}(10)$ & $0.948(19)$ & $\mathrm{C}(11)-\mathrm{H}(11)$ & $0.965(18)$ \\
\hline $\mathrm{C}(12)-\mathrm{C}(17)$ & $1.386(2)$ & $\mathrm{C}(12)-\mathrm{C}(13)$ & $1.392(2)$ \\
\hline C(13)-C(14) & $1.389(2)$ & $\mathrm{C}(13)-\mathrm{H}(12)$ & $0.965(16)$ \\
\hline C(14)-C(15) & $1.382(2)$ & $\mathrm{C}(14)-\mathrm{H}(13)$ & $0.965(19)$ \\
\hline C(15)-C(16) & $1.376(2)$ & $\mathrm{C}(15)-\mathrm{H}(14)$ & $0.971(18)$ \\
\hline C(16)-C(17) & $1.395(2)$ & $\mathrm{C}(16)-\mathrm{H}(15)$ & $0.973(19)$ \\
\hline $\mathrm{C}(17)-\mathrm{H}(16)$ & $0.965(17)$ & $\mathrm{C}(19)-\mathrm{H}(17)$ & $0.95(2)$ \\
\hline C(19)-H(19) & $0.93(3)$ & $\mathrm{C}(19)-\mathrm{H}(18)$ & $1.03(3)$ \\
\hline $\mathrm{C}(18)-\mathrm{O}(2)-\mathrm{C}(19)$ & $\mathrm{C}(1)-\mathrm{N}(1)-\mathrm{C}(5)$ & $122.85(13)$ & \\
\hline $\mathrm{C}(1)-\mathrm{N}(1)-\mathrm{C}(4)$ & $\mathrm{C}(5)-\mathrm{N}(1)-\mathrm{C}(4)$ & $121.29(12)$ & \\
\hline $\mathrm{O}(1)-\mathrm{C}(1)-\mathrm{N}(1)$ & $\mathrm{O}(1)-\mathrm{C}(1)-\mathrm{C}(2)$ & 126.61(14) & \\
\hline $\mathrm{N}(1)-\mathrm{C}(1)-\mathrm{C}(2)$ & $C(1)-C(2)-C(3)$ & $105.45(12)$ & \\
\hline $\mathrm{C}(1)-\mathrm{C}(2)-\mathrm{H}(2)$ & $\mathrm{C}(3)-\mathrm{C}(2)-\mathrm{H}(2)$ & 112.3(9) & \\
\hline $\mathrm{C}(1)-\mathrm{C}(2)-\mathrm{H}(1)$ & $\mathrm{C}(3)-\mathrm{C}(2)-\mathrm{H}(1)$ & 112.5(9) & \\
\hline $\mathrm{H}(2)-\mathrm{C}(2)-\mathrm{H}(1)$ & $\mathrm{C}(18)-\mathrm{C}(3)-\mathrm{C}(2)$ & 114.18(13) & \\
\hline $\mathrm{C}(18)-\mathrm{C}(3)-\mathrm{C}(4)$ & $C(2)-C(3)-C(4)$ & $105.14(12)$ & \\
\hline $\mathrm{C}(18)-\mathrm{C}(3)-\mathrm{H}(3)$ & $\mathrm{C}(2)-\mathrm{C}(3)-\mathrm{H}(3)$ & 111.1(9) & \\
\hline $\mathrm{C}(4)-\mathrm{C}(3)-\mathrm{H}(3)$ & $\mathrm{N}(1)-\mathrm{C}(4)-\mathrm{C}(12)$ & 111.67(11) & \\
\hline $\mathrm{N}(1)-\mathrm{C}(4)-\mathrm{C}(3)$ & $\mathrm{C}(12)-\mathrm{C}(4)-\mathrm{C}(3)$ & $116.52(11)$ & \\
\hline
\end{tabular}




$\begin{array}{lll}\mathrm{N}(1)-\mathrm{C}(4)-\mathrm{H}(4) & \mathrm{C}(12)-\mathrm{C}(4)-\mathrm{H}(4) & 108.4(8) \\ \mathrm{C}(3)-\mathrm{C}(4)-\mathrm{H}(4) & \mathrm{N}(1)-\mathrm{C}(5)-\mathrm{C}(6) & 112.96(12) \\ \mathrm{N}(1)-\mathrm{C}(5)-\mathrm{H}(5) & \mathrm{C}(6)-\mathrm{C}(5)-\mathrm{H}(5) & 109.6(9) \\ \mathrm{N}(1)-\mathrm{C}(5)-\mathrm{H}(6) & \mathrm{C}(6)-\mathrm{C}(5)-\mathrm{H}(6) & 112.6(9) \\ \mathrm{H}(5)-\mathrm{C}(5)-\mathrm{H}(6) & \mathrm{C}(11)-\mathrm{C}(6)-\mathrm{C}(7) & 118.81(15) \\ \mathrm{C}(11)-\mathrm{C}(6)-\mathrm{C}(5) & \mathrm{C}(7)-\mathrm{C}(6)-\mathrm{C}(5) & 121.32(13) \\ \mathrm{C}(8)-\mathrm{C}(7)-\mathrm{C}(6) & \mathrm{C}(8)-\mathrm{C}(7)-\mathrm{H}(7) & 119.5(9) \\ \mathrm{C}(6)-\mathrm{C}(7)-\mathrm{H}(7) & \mathrm{C}(7)-\mathrm{C}(8)-\mathrm{C}(9) & 120.35(16) \\ \mathrm{C}(7)-\mathrm{C}(8)-\mathrm{H}(8) & \mathrm{C}(9)-\mathrm{C}(8)-\mathrm{H}(8) & 119.3(11) \\ \mathrm{C}(10)-\mathrm{C}(9)-\mathrm{C}(8) & \mathrm{C}(10)-\mathrm{C}(9)-\mathrm{H}(9) & 120.8(11) \\ \mathrm{C}(8)-\mathrm{C}(9)-\mathrm{H}(9) & \mathrm{C}(9)-\mathrm{C}(10)-\mathrm{C}(11) & 120.31(16) \\ \mathrm{C}(9)-\mathrm{C}(10)-\mathrm{H}(10) & \mathrm{C}(11)-\mathrm{C}(10)-\mathrm{H}(10) & 118.1(11) \\ \mathrm{C}(10)-\mathrm{C}(11)-\mathrm{C}(6) & \mathrm{C}(10)-\mathrm{C}(11)-\mathrm{H}(11) & 120.3(11) \\ \mathrm{C}(6)-\mathrm{C}(11)-\mathrm{H}(11) & \mathrm{C}(17)-\mathrm{C}(12)-\mathrm{C}(13) & 118.66(13) \\ \mathrm{C}(17)-\mathrm{C}(12)-\mathrm{C}(4) & \mathrm{C}(13)-\mathrm{C}(12)-\mathrm{C}(4) & 121.45(13) \\ \mathrm{C}(14)-\mathrm{C}(13)-\mathrm{C}(12) & \mathrm{C}(14)-\mathrm{C}(13)-\mathrm{H}(12) & 118.9(9) \\ \mathrm{C}(12)-\mathrm{C}(13)-\mathrm{H}(12) & \mathrm{C}(15)-\mathrm{C}(14)-\mathrm{C}(13) & 119.96(16) \\ \mathrm{C}(15)-\mathrm{C}(14)-\mathrm{H}(13) & \mathrm{C}(13)-\mathrm{C}(14)-\mathrm{H}(13) & 118.9(11) \\ \mathrm{C}(16)-\mathrm{C}(15)-\mathrm{C}(14) & \mathrm{C}(16)-\mathrm{C}(15)-\mathrm{H}(14) & 120.1(11) \\ \mathrm{C}(14)-\mathrm{C}(15)-\mathrm{H}(14) & \mathrm{C}(15)-\mathrm{C}(16)-\mathrm{C}(17) & 119.86(15) \\ \mathrm{C}(15)-\mathrm{C}(16)-\mathrm{H}(15) & \mathrm{C}(17)-\mathrm{C}(16)-\mathrm{H}(15) & 119.9(11) \\ \mathrm{C}(12)-\mathrm{C}(17)-\mathrm{C}(16) & \mathrm{C}(12)-\mathrm{C}(17)-\mathrm{H}(16) & 119.4(10) \\ \mathrm{C}(16)-\mathrm{C}(17)-\mathrm{H}(16) & \mathrm{O}(3)-\mathrm{C}(18)-\mathrm{O}(2) & 123.06(14) \\ \mathrm{O}(3)-\mathrm{C}(18)-\mathrm{C}(3) & \mathrm{O}(2)-\mathrm{C}(18)-\mathrm{C}(3) & 111.58(13) \\ \mathrm{O}(2)-\mathrm{C}(19)-\mathrm{H}(17) & \mathrm{O}(2)-\mathrm{C}(19)-\mathrm{H}(19) & 110.4(15) \\ \mathrm{H}(17)-\mathrm{C}(19)-\mathrm{H}(19) & \mathrm{O}(2)-\mathrm{C}(19)-\mathrm{H}(18) & 107.4(15) \\ \mathrm{H}(17)-\mathrm{C}(19)-\mathrm{H}(18) & \mathrm{H}(19)-\mathrm{C}(19)-\mathrm{H}(18) & \\ & & \\ & & \end{array}$

Symmetry transformations used to generate equivalent atoms: 
Table 4. Anisotropic displacement parameters $\left(\AA^{2} \times 10^{3}\right)$ for jts06t. The anisotropic displacement factor exponent takes the form: $-2 \pi^{2}\left[h^{2} a^{* 2} U^{11}+\ldots+2 h \mathrm{k}^{*} b^{*} U^{12}\right]$

\begin{tabular}{|c|c|c|c|c|c|c|}
\hline & $\mathrm{U}^{11}$ & $\mathrm{U}^{22}$ & $\mathrm{U}^{33}$ & $\mathrm{U}^{23}$ & $\mathrm{U}^{13}$ & $\mathrm{U}^{12}$ \\
\hline $\mathrm{O}(1)$ & $33(1)$ & 26(1) & 39(1) & $1(1)$ & 13(1) & $-2(1)$ \\
\hline $\mathrm{O}(2)$ & 29(1) & $26(1)$ & $56(1)$ & $-1(1)$ & $1(1)$ & $-1(1)$ \\
\hline $\mathrm{O}(3)$ & $32(1)$ & $34(1)$ & $56(1)$ & $6(1)$ & $0(1)$ & 2(1) \\
\hline $\mathrm{N}(1)$ & $24(1)$ & $27(1)$ & $25(1)$ & $-1(1)$ & $6(1)$ & $-2(1)$ \\
\hline $\mathrm{C}(1)$ & 23(1) & $25(1)$ & $30(1)$ & $0(1)$ & $12(1)$ & $5(1)$ \\
\hline$C(2)$ & $29(1)$ & $31(1)$ & $25(1)$ & $2(1)$ & $8(1)$ & 1(1) \\
\hline C(3) & $26(1)$ & $26(1)$ & $26(1)$ & $-3(1)$ & $8(1)$ & $4(1)$ \\
\hline$C(4)$ & 23(1) & $24(1)$ & $26(1)$ & $1(1)$ & $5(1)$ & 1(1) \\
\hline C(5) & $29(1)$ & $30(1)$ & $28(1)$ & $-5(1)$ & $6(1)$ & $-1(1)$ \\
\hline$C(6)$ & $22(1)$ & $31(1)$ & $27(1)$ & $-3(1)$ & $3(1)$ & $-5(1)$ \\
\hline C(7) & $28(1)$ & $37(1)$ & 29(1) & $-5(1)$ & $4(1)$ & $0(1)$ \\
\hline C(8) & $32(1)$ & $39(1)$ & $41(1)$ & $-9(1)$ & $2(1)$ & $3(1)$ \\
\hline C(9) & $34(1)$ & $39(1)$ & $46(1)$ & $5(1)$ & $-4(1)$ & $3(1)$ \\
\hline$C(10)$ & $39(1)$ & $54(1)$ & 29(1) & $6(1)$ & $1(1)$ & $-3(1)$ \\
\hline $\mathrm{C}(11)$ & $31(1)$ & $45(1)$ & 29(1) & $-3(1)$ & $9(1)$ & $-1(1)$ \\
\hline$C(12)$ & $23(1)$ & 29(1) & $22(1)$ & $-2(1)$ & $3(1)$ & $-3(1)$ \\
\hline$C(13)$ & $34(1)$ & $30(1)$ & $32(1)$ & $2(1)$ & $12(1)$ & 1(1) \\
\hline$C(14)$ & $33(1)$ & $40(1)$ & $41(1)$ & $-4(1)$ & $11(1)$ & $4(1)$ \\
\hline$C(15)$ & $32(1)$ & $51(1)$ & $34(1)$ & $-4(1)$ & $14(1)$ & $-5(1)$ \\
\hline$C(16)$ & $42(1)$ & $45(1)$ & $35(1)$ & $7(1)$ & $15(1)$ & $-6(1)$ \\
\hline$C(17)$ & $34(1)$ & $32(1)$ & $34(1)$ & $4(1)$ & $9(1)$ & $1(1)$ \\
\hline$C(18)$ & $34(1)$ & 26(1) & $25(1)$ & $-4(1)$ & $7(1)$ & 2(1) \\
\hline C(19) & $31(1)$ & $31(1)$ & $95(2)$ & $-2(1)$ & $-3(1)$ & $-5(1)$ \\
\hline
\end{tabular}


Table 5. Hydrogen coordinates ( x 104) and isotropic displacement parameters $\left(\AA^{2} \times 10^{3}\right)$ for jts06t.

\begin{tabular}{|c|c|c|c|c|}
\hline & $\mathrm{x}$ & $\mathrm{y}$ & $\mathrm{z}$ & $\mathrm{U}(\mathrm{eq})$ \\
\hline $\mathrm{H}(2)$ & $8557(12)$ & $4230(30)$ & $-675(8)$ & $32(4)$ \\
\hline $\mathrm{H}(1)$ & $7669(12)$ & $5280(30)$ & $-393(8)$ & $26(4)$ \\
\hline $\mathrm{H}(3)$ & $8457(11)$ & $830(30)$ & $-171(7)$ & 23(4) \\
\hline $\mathrm{H}(4)$ & 8825(10) & $730(20)$ & 930(7) & $17(3)$ \\
\hline $\mathrm{H}(5)$ & $9231(12)$ & $5170(30)$ & $1824(8)$ & $32(4)$ \\
\hline $\mathrm{H}(6)$ & $10061(12)$ & $6160(30)$ & 1466(8) & $37(5)$ \\
\hline $\mathrm{H}(7)$ & $10720(11)$ & $1550(30)$ & 993(9) & 33(4) \\
\hline $\mathrm{H}(8)$ & 11825(13) & $-1330(30)$ & 1546(9) & $44(5)$ \\
\hline $\mathrm{H}(9)$ & 12126(13) & $-1780(30)$ & 2722(9) & $42(5)$ \\
\hline $\mathrm{H}(10)$ & 11344(13) & $700(30)$ & $3357(10)$ & $45(5)$ \\
\hline $\mathrm{H}(11)$ & 10256(13) & $3530(30)$ & 2822(9) & $47(5)$ \\
\hline $\mathrm{H}(12)$ & 7133(11) & $5560(30)$ & 819(8) & $32(4)$ \\
\hline $\mathrm{H}(13)$ & $5870(14)$ & $5840(30)$ & $1400(9)$ & $46(5)$ \\
\hline $\mathrm{H}(14)$ & $5622(13)$ & $2890(30)$ & 2135(9) & $45(5)$ \\
\hline $\mathrm{H}(15)$ & $6645(14)$ & $-380(30)$ & 2263(9) & $49(5)$ \\
\hline $\mathrm{H}(16)$ & $7873(12)$ & $-740(30)$ & 1629(8) & $37(5)$ \\
\hline $\mathrm{H}(17)$ & 5921(14) & $-3540(40)$ & $-369(10)$ & $58(6)$ \\
\hline H(19) & $5514(18)$ & $-1250(40)$ & $-742(13)$ & $78(8)$ \\
\hline $\mathrm{H}(18)$ & $5520(20)$ & $-1570(50)$ & $42(15)$ & 109(10) \\
\hline
\end{tabular}


Table 6. Torsion angles $\left[{ }^{\circ}\right]$ for jts06t.

\begin{tabular}{|c|c|}
\hline $\mathrm{C}(5)-\mathrm{N}(1)-\mathrm{C}(1)-\mathrm{O}(1)$ & $3.1(2)$ \\
\hline $\mathrm{C}(4)-\mathrm{N}(1)-\mathrm{C}(1)-\mathrm{O}(1)$ & $175.57(12)$ \\
\hline C(5)-N(1)-C(1)-C(2) & $-175.72(12)$ \\
\hline $\mathrm{C}(4)-\mathrm{N}(1)-\mathrm{C}(1)-\mathrm{C}(2)$ & $-3.26(16)$ \\
\hline $\mathrm{O}(1)-\mathrm{C}(1)-\mathrm{C}(2)-\mathrm{C}(3)$ & $171.54(13)$ \\
\hline $\mathrm{N}(1)-\mathrm{C}(1)-\mathrm{C}(2)-\mathrm{C}(3)$ & $-9.65(15)$ \\
\hline C(1)-C(2)-C(3)-C(18) & $141.61(12)$ \\
\hline $\mathrm{C}(1)-\mathrm{C}(2)-\mathrm{C}(3)-\mathrm{C}(4)$ & $17.63(14)$ \\
\hline $\mathrm{C}(1)-\mathrm{N}(1)-\mathrm{C}(4)-\mathrm{C}(12)$ & $-111.00(14)$ \\
\hline $\mathrm{C}(5)-\mathrm{N}(1)-\mathrm{C}(4)-\mathrm{C}(12)$ & $61.59(16)$ \\
\hline $\mathrm{C}(1)-\mathrm{N}(1)-\mathrm{C}(4)-\mathrm{C}(3)$ & $14.15(15)$ \\
\hline C(5)-N(1)-C(4)-C(3) & $-173.26(12)$ \\
\hline $\mathrm{C}(18)-\mathrm{C}(3)-\mathrm{C}(4)-\mathrm{N}(1)$ & $-143.74(12)$ \\
\hline $\mathrm{C}(2)-\mathrm{C}(3)-\mathrm{C}(4)-\mathrm{N}(1)$ & $-18.80(14)$ \\
\hline C(18)-C(3)-C(4)-C(12) & $-21.87(18)$ \\
\hline C(2)-C(3)-C(4)-C(12) & 103.07(14) \\
\hline $\mathrm{C}(1)-\mathrm{N}(1)-\mathrm{C}(5)-\mathrm{C}(6)$ & $-130.19(14)$ \\
\hline $\mathrm{C}(4)-\mathrm{N}(1)-\mathrm{C}(5)-\mathrm{C}(6)$ & $57.78(17)$ \\
\hline $\mathrm{N}(1)-\mathrm{C}(5)-\mathrm{C}(6)-\mathrm{C}(11)$ & $-144.20(14)$ \\
\hline N(1)-C(5)-C(6)-C(7) & 36.01(19) \\
\hline C(11)-C(6)-C(7)-C(8) & $-0.3(2)$ \\
\hline C(5)-C(6)-C(7)-C(8) & 179.51(14) \\
\hline C(6)-C(7)-C(8)-C(9) & $0.6(2)$ \\
\hline C(7)-C(8)-C(9)-C(10) & $-0.7(3)$ \\
\hline C(8)-C(9)-C(10)-C(11) & $0.4(3)$ \\
\hline C(9)-C(10)-C(11)-C(6) & $-0.1(3)$ \\
\hline C(7)-C(6)-C(11)-C(10) & $0.0(2)$ \\
\hline$C(5)-C(6)-C(11)-C(10)$ & $-179.77(14)$ \\
\hline N(1)-C(4)-C(12)-C(17) & $-128.45(15)$ \\
\hline$C(3)-C(4)-C(12)-C(17)$ & $114.91(16)$ \\
\hline N(1)-C(4)-C(12)-C(13) & $50.39(18)$ \\
\hline C(3)-C(4)-C(12)-C(13) & $-66.25(18)$ \\
\hline C(17)-C(12)-C(13)-C(14) & $-1.2(2)$ \\
\hline C(4)-C(12)-C(13)-C(14) & 179.91(14) \\
\hline
\end{tabular}




\begin{tabular}{lc}
$\mathrm{C}(12)-\mathrm{C}(13)-\mathrm{C}(14)-\mathrm{C}(15)$ & $0.9(3)$ \\
$\mathrm{C}(13)-\mathrm{C}(14)-\mathrm{C}(15)-\mathrm{C}(16)$ & $0.1(3)$ \\
$\mathrm{C}(14)-\mathrm{C}(15)-\mathrm{C}(16)-\mathrm{C}(17)$ & $-0.8(3)$ \\
$\mathrm{C}(13)-\mathrm{C}(12)-\mathrm{C}(17)-\mathrm{C}(16)$ & $0.5(2)$ \\
$\mathrm{C}(4)-\mathrm{C}(12)-\mathrm{C}(17)-\mathrm{C}(16)$ & $179.39(15)$ \\
$\mathrm{C}(15)-\mathrm{C}(16)-\mathrm{C}(17)-\mathrm{C}(12)$ & $0.5(3)$ \\
$\mathrm{C}(19)-\mathrm{O}(2)-\mathrm{C}(18)-\mathrm{O}(3)$ & $0.0(2)$ \\
$\mathrm{C}(19)-\mathrm{O}(2)-\mathrm{C}(18)-\mathrm{C}(3)$ & $179.37(16)$ \\
$\mathrm{C}(2)-\mathrm{C}(3)-\mathrm{C}(18)-\mathrm{O}(3)$ & $-13.4(2)$ \\
$\mathrm{C}(4)-\mathrm{C}(3)-\mathrm{C}(18)-\mathrm{O}(3)$ & $106.47(17)$ \\
$\mathrm{C}(2)-\mathrm{C}(3)-\mathrm{C}(18)-\mathrm{O}(2)$ & $167.23(12)$ \\
$\mathrm{C}(4)-\mathrm{C}(3)-\mathrm{C}(18)-\mathrm{O}(2)$ & $-72.92(16)$ \\
& \\
\hline
\end{tabular}

Symmetry transformations used to generate equivalent atoms: 